\title{
Decay predictions for the Georgi-Machacek extended Higgs particle model
}

\author{
by
}

Mark Reimer

\begin{abstract}
A thesis submitted to the Faculty of Graduate and Postdoctoral Affairs in partial fulfillment of the requirements for the degree of

Master of Science

in

Physics
\end{abstract}

Ottawa-Carleton Institute for Physics

Carleton University

Ottawa, Ontario

(C) 2018

Mark Reimer 


\section{Abstract}

The Georgi-Machacek (GM) model is a theoretical particle model which hypothesizes the existence of additional Higgs particles, the most notable of which is a Higgs particle with a double electric charge $(+2 \mathrm{e})$. The GM model thus serves as a useful prototype for determining the experimental behaviour of models which contain doubly-charged Higgs particles. To aid in the search for such particles, we have uti-

lized the public code GMCALC to conduct a systematic study of a benchmark for the GM model. We have computed a complete set of predictions for particle behaviour, primarily decay rates and branching ratios. We have also made improvements to the GMCALC code so that it will be able to compute decay rates more accurately and efficiently in the low-scalar-mass region of parameter space. We will present these results below. 


\section{Acknowledgements}

I would like to thank my supervisor, Heather Logan, for all her teaching, instruction, and assistance in this project. Thanks to the authors of GMCALC for constructing a highly flexible computational tool for the Georgi-Machacek model. Finally, thanks to Carleton University for enabling me to pursue this research endeavour. 


\section{Statement of originality}

In the course of my studies, I have written programs which utilized the GMCALC code to compute predictions for the behaviour of the GM model, converted the resulting data into figures and graphs for ease of interpretation, and built, tested, and optimized code that improves the accuracy and efficiency of certain offshell decay calculations in the GMCALC code. Some of the results of this work have appeared in [1]. I certify that the intellectual content of this thesis is my own work and that all assistance received and sources used in preparing this thesis have been acknowledged. 


\section{Contents}

Abstract $\quad$ ii

Acknowledgements iii

Statement of originality iv

List of Tables $\quad$ vii

List of Figures $\quad$ X

List of Appendices $\quad$ xi

1 Introduction 1

1.1 The Standard Model . . . . . . . . . . . . . . . . . . . . . 2

1.2 Feynman diagrams, branching ratios, and decay widths . . . . . . . 3

1.3 Fermions and chiral symmetry . . . . . . . . . . . . . . . 7

1.4 Gauge symmetry . . . . . . . . . . . . . . . . . . . . . . . 8

1.5 Electroweak interactions . . . . . . . . . . . . . . . . . 11

1.6 Higgs mechanism . . . . . . . . . . . . . . . . . . . . . . . 13

2 The Georgi-Machacek model, GMCALC, and the H5plane bench$\begin{array}{ll}\text { mark } & 17\end{array}$

2.1 The Georgi-Machacek model . . . . . . . . . . . . . . . . . . . 18

2.2 GMCALC . . . . . . . . . . . . . . . . . . . . . . 21

2.3 H5plane benchmark . . . . . . . . . . . . . . . . . 23

2.4 Constraints on the H5plane benchmark . . . . . . . . . . . . . . . 25

3 Properties of the H5plane benchmark 29

3.1 Decays of $H_{5} \quad \ldots \ldots \ldots \ldots$

$3.2 \quad H_{3}-H_{5}$ mass splitting . . . . . . . . . . . . . . . . . . 32 
3.3 Couplings and decays of $h \ldots \ldots$. . . . . . . . . . . . 35

3.4 Couplings and decays of $H \ldots \ldots$. . . . . . . . . . . . 47

$3.5 \quad H-H_{5}$ mass splitting . . . . . . . . . . . . . . . . . . . 49

3.6 Decays of $H_{3} \ldots \ldots \ldots \ldots \ldots \ldots$

4 Offshell decay algorithms $\quad 74$

$4.1 \quad H_{i} \rightarrow V_{1}^{*} V_{2}^{*}$ integration routine . . . . . . . . . . . . . 74

4.2 Gauss-Legendre quadrature . . . . . . . . . . . . . . . . 82

4.3 The VEGAS routine . . . . . . . . . . . . . . . 85

4.4 Final integration routine . . . . . . . . . . . . . . . 90

5 Conclusions $\quad 94$

$\begin{array}{lr}\text { References } & 98\end{array}$

$\begin{array}{ll}\text { Appendices } & 102\end{array}$

Appendix A: Gauss-Legendre quadrature . . . . . . . . . . . . . . . . 102 


\section{List of Tables}

1 Standard Model . . . . . . . . . . . . . . . . . . . 3

2 Higgs particles in the GM model . . . . . . . . . . . . . . . . 17

3 H5plane benchmark parameters ................ 24

4 Branching ratios of the SM Higgs boson with mass $125.09 \mathrm{GeV}$. . . . 42

$5 \quad$ Nodes and weights of 16-point Gauss-Legendre quadrature . . . . . . 104 


\section{List of Figures}

1 Feynman diagrams for electron-positron scattering . . . . . . . . . . 4

2 Particle mass shell . . . . . . . . . . . . . . . . . . 5

3 Allowed parameter region for H5plane . . . . . . . . . . . 26

4 Contours of $\Gamma_{\text {tot }} / m_{5}$ for $H_{5}^{++} \ldots \ldots \ldots$. . . . . . . . . 30

5 Deviation of total widths of $H_{5}^{+}$and $H_{5}^{0}$ from that of $H_{5}^{++} \ldots . . .31$

6 Contours of $m_{3}-m_{5}$ in the H5plane benchmark . . . . . . . . . . . . 32

$7 \quad$ Mass difference $m_{3}-m_{5}$ in the H5plane benchmark and in a full scan 33

8 Contours of $\sin \alpha$ in the H5plane benchmark . . . . . . . . . . . . 36

9 Correlation between $\sin \alpha$ and $s_{H}$ in the H5plane benchmark and in a full scan . . . . . . . . . . . . . . . . . . . . . . . 37

10 Contours of $\kappa_{f}^{h}$ in the H5plane benchmark . . . . . . . . . . . . 38

11 Contours of $\kappa_{V}^{h}$ in the H5plane benchmark . . . . . . . . . . . . 39

12 Contours of $\kappa_{\gamma}^{h}$ in the H5plane benchmark. . . . . . . . . . . . . . 40

13 Contours of $\Delta \kappa_{\gamma}^{h}$ in the H5plane benchmark. . . . . . . . . . . . . 41

14 Contours of $\kappa_{h}$ in the H5plane benchmark . . . . . . . . . . . . 43

$15 \kappa_{h}$ in the H5plane benchmark and in a full scan . . . . . . . . . . 44

16 Contours of the $\chi^{2}$ value for a fit of the $h$ data in the H5plane benchmark 46

17 Contours of the $\chi^{2}$ value for a modified fit of the $h$ data in the H5plane benchmark. . . . . . . . . . . . . . . . . 47

18 Contours of $\kappa_{f}^{H}$ in the H5plane benchmark . . . . . . . . . . . . 49

19 Contours of $\kappa_{V}^{H}$ in the H5plane benchmark . . . . . . . . . . . . 50

20 Contours of $\mathrm{BR}\left(H \rightarrow W^{+} W^{-}\right)$in the H5plane benchmark . . . . . 51

21 Contours of $\mathrm{BR}(H \rightarrow Z Z)$ in the H5plane benchmark . . . . . . . . 52

22 Contours of $\mathrm{BR}(H \rightarrow h h)$ in the H5plane benchmark . . . . . . . 53

23 Contours of $\mathrm{BR}(H \rightarrow t \bar{t})$ in the H5plane benchmark. . . . . . . . 54

24 Contours of $m_{H}-m_{5}$ in the H5plane benchmark . . . . . . . . 55 
25 Mass difference $m_{H}-m_{5}$ in the H5plane benchmark and in a full scan 56

26 Contours of $\Gamma_{\text {tot }}(H)$ in the H5plane benchmark . . . . . . . . . 58

27 Contours of the ratio $\Gamma_{\text {tot }}(H) / \Gamma_{\text {tot }}\left(H_{5}^{0}\right)$ in the H5plane benchmark . . 59

28 Contours of $\left(m_{H}-m_{5}\right) / \Gamma_{\text {tot }}(H)$ in the H5plane benchmark . . . . . . 60

29 Contours of $\mathrm{BR}\left(H_{3}^{0} \rightarrow t \bar{t}\right)$ in the H5plane benchmark . . . . . . . . 61

30 Contours of $\mathrm{BR}\left(H_{3}^{0} \rightarrow h Z\right)$ in the H5plane benchmark . . . . . . . 62

31 Contours of $\mathrm{BR}\left(H_{3}^{0} \rightarrow H_{5}^{0} Z\right)$ in the H5plane benchmark . . . . . 64

32 Contours of $\operatorname{BR}\left(H_{3}^{0} \rightarrow H_{5}^{+} W^{-}+H_{5}^{-} W^{+}\right)$in the H5plane benchmark 65

33 Contours of $\mathrm{BR}\left(H_{3}^{+} \rightarrow t \bar{b}\right)$ in the H5plane benchmark . . . . . . . . 66

34 Contours of $\mathrm{BR}\left(H_{3}^{+} \rightarrow h W^{+}\right)$in the H5plane benchmark . . . . . . 67

35 Contours of $\mathrm{BR}\left(H_{3}^{+} \rightarrow H_{5}^{0} W^{+}\right)$in the H5plane benchmark . . . . . . 68

36 Contours of $\mathrm{BR}\left(H_{3}^{+} \rightarrow H_{5}^{+} Z\right)$ in the H5plane benchmark . . . . . . 69

37 Contours of $\mathrm{BR}\left(H_{3}^{+} \rightarrow H_{5}^{++} W^{-}\right)$in the H5plane benchmark . . . . . 70

38 Contours of $\Gamma_{\text {tot }}\left(H_{3}^{0}\right) / m_{3}$ in the H5plane benchmark . . . . . . . . . . 71

39 Contours of $\Gamma_{\text {tot }}\left(H_{3}^{+}\right) / m_{3}$ in the H5plane benchmark . . . . . . . . 72

40 Integrand with respect to $Q_{i}$ for $H_{i} \rightarrow W^{+} W^{-}$with $m_{H_{i}}=0.9 M_{W}$. . 76

41 Integrand with respect to $\rho_{i}$ for $H_{i} \rightarrow W^{+} W^{-}$with $m_{H_{i}}=0.9 M_{W}$. . 77

42 Integrand with respect to $Q_{i}$ for $H_{i} \rightarrow W^{+} W^{-}$with $m_{H_{i}}=1.5 M_{W}$. . 78

43 Integrand with respect to $\rho_{i}$ for $H_{i} \rightarrow W^{+} W^{-}$with $m_{H_{i}}=1.5 M_{W}$. . 79

44 Integrand with respect to $Q_{i}$ for $H_{i} \rightarrow W^{+} W^{-}$with $m_{H_{i}}=2.1 M_{W}$. . 80

45 Integrand with respect to $\rho_{i}$ for $H_{i} \rightarrow W^{+} W^{-}$with $m_{H_{i}}=2.1 M_{W}$. . 81

46 Relative error of $H_{i} \rightarrow W^{+} W^{-}$integration routines compared to HDECAY $6.42 \ldots \ldots \ldots \ldots \ldots$. . . . . . . . . . . . . . . 83

47 Relative error of $H_{i} \rightarrow Z Z$ integration routines compared to HDECAY $6.42 \ldots \ldots \ldots \ldots \ldots$. . . . . . . . . . . . . . . 84

48 Relative error of $\Gamma\left(H_{i} \rightarrow W^{+} W^{-}\right)$VEGAS routine compared to HDECAY 6.42 and original . . . . . . . . . . . . . . . 87 
49 Optimizing relative error of $\Gamma\left(H_{i} \rightarrow V_{1} V_{2}\right)$ VEGAS routine compared to original . . . . . . . . . . . . . . . . . . . 88

50 Optimizing relative error of $\Gamma\left(H_{i} \rightarrow V_{1} V_{2}\right)$ VEGAS routine compared to itself . . . . . . . . . . . . . . . . . . 89

51 Time taken to run VEGAS routine . . . . . . . . . . . . . . . 90

52 Relative error of $\Gamma\left(H_{i} \rightarrow W W\right)$ compared to HDECAY in original and final routine ...................... . . . . . 91

53 Relative error of $\Gamma\left(H_{i} \rightarrow Z Z\right)$ compared to HDECAY in original and final routine . . . . . . . . . . . . . . . . . . 92

54 Timing of original and final routines f . . . . . . . . . . . . . 93 


\section{List of Appendices}

Appendix A: Gauss-Legendre quadrature . . . . . . . . . . . . . . . . . . 102 


\section{Introduction}

The Standard Model of particle physics is one of the most well-tested theories we have, and through it we have been able to explain many of the phenomena we observe in the universe, from condensed matter physics to cosmic ray showers to the fusion within stars. However, there are still areas where the Standard Model falls short: it cannot explain the existence of dark matter, which we can observe through its gravitational pull on galaxies but have been unable to detect through non-gravitational means; it leaves unsolved the matter-antimatter asymmetry problem, which enables normal matter to exist in such abundance in the universe; and, at the smallest scales and the highest energies, it yields divergent and nonsensical results, proving its own inadequacy in providing a unifying explanation for the behaviour and origin of the universe. In summary, the Standard Model is an incomplete picture of particle behaviour, and we are obligated to search for the physics which lies beyond it.

However, before we can search for evidence of new physics in the world, we must first be able to recognize the entities for which we are searching. The high-energy particle collisions at the Large Hadron Collider (LHC) produce massive amounts of data - so much data, in fact, that it is impossible to keep track of it all. As a result, the computers which analyze the data must choose what records to keep and what to delete. If one wishes to find, say, a new kind of scalar particle, one must first know how such a particle is likely to behave so that one can look for such behaviour in the detectors.

This is where the Georgi-Machacek (GM) model comes in. While there are many potential new particles which could exist and need to be searched for, the GM model specifically proposes the existence of additional Higgs particles. In particular, one of the new particles it proposes is a doubly-charged scalar (that is, a particle with zero intrinsic angular momentum and an electric charge that is twice as big as an electron's). While there exist other models which propose the existence of such a 
particle, the GM model is among the simplest. As such, the GM model serves as a useful prototype for predicting how a doubly-charged scalar particle should behave, and how we could detect it if it exists. It is for this reason that we have chosen to study the Georgi-Machacek model, with the purpose of deriving predictions for the behaviour of its particles and improving the efficiency of the tools used in those predictions.

The thesis is organized as follows: In this first section, we cover some preliminary particle theory, to give some necessary background for the later sections. In Section 2, we discuss the Georgi-Machacek model and the tools we will be using to analyze it. Among these tools are the GMCALC code, which will be used for the computation of our predictions, and the H5plane benchmark scenario, which reduces the size of the parameter space we must scan in order to predict the full range of possible particle behaviour. Section 3 contains the predictions we have derived for the behaviour of the GM model within the H5plane benchmark, and Section 4 will discuss improvements we have made to the GMCALC code in order to make it more efficient for future use. Finally, in Section 5 we will sum up our results and propose future directions for the research contained in this paper.

\subsection{The Standard Model}

The Standard Model (SM) is the theory which describes all of the particle physics which we have been able to explain so far. The collection of all fundamental particles in the Standard Model are listed in Table 1. We have: the quarks, fermions that are subject to the strong nuclear force; the leptons, fermions that are not subject to the strong nuclear force; the gauge bosons, which communicate three of the four fundamental forces; and the Higgs boson, which causes particles to have mass.

The fermions can be broken into three generations, with each generation being composed of progressively heavier particles. Taking electric charges to be normalized 
such that the electron has a charge of -1 , each generation has a charge $+2 / 3$ quark $(u, c, t)$, a charge $-1 / 3$ quark $(d, s, b)$, a charge -1 lepton $(e, \mu, \tau)$, and a low-mass, zero-charge neutrino $\left(\nu_{e}, \nu_{\mu}, \nu_{\tau}\right)$. The gauge bosons (which are not divided into generations) communicate three fundamental forces: the gluon, $g$, communicates the strong nuclear force and binds together the nuclei of atoms; the photon, $\gamma$, communicates the electromagnetic force; and the $W$ and $Z$ bosons communicate the weak nuclear force (which is responsible for certain kinds of radioactive decay). The behaviour of all these particles, and any particles yet to be discovered, can be analyzed through the lens of an extraordinarily useful tool: Feynman diagrams.

\subsection{Feynman diagrams, branching ratios, and decay widths}

When considering the likelihood that a given particle event will occur, whether it is the scattering of particles off of each other, the decay of one particle into multiple particles, or the production of new particles in a particle collider, one can interpret such an event as the superposition of many different particle interactions, which are represented by Feynman diagrams of increasing complexity. For example, consider the event of an electron and a positron scattering off of each other. Several of the Feynman diagrams associated with this event are shown in Fig. 1. Each line in the diagram represents a particle, and each vertex represents an interaction between particles. Fermions are represented by lines with a forward-facing arrow, antifermions are represented by lines with a backward-facing arrow, and photons are represented

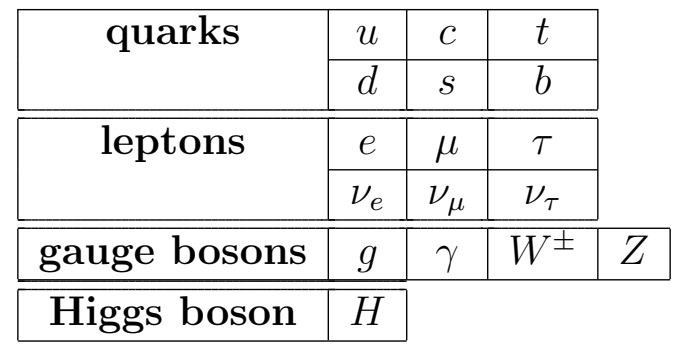

Table 1: All fundamental particles in the Standard Model 
by wavy lines. As a general rule, the more interaction vertices a Feynman diagram has, the less it contributes to the total probability of a given event. In addition, if the Feynman diagrams are considered as graphs, with the interaction vertices being graph nodes and the particle lines being edges connecting the nodes, then one sees that the two rightmost diagrams each contain a closed path, or loop (i.e. you can start at one vertex, travel along the internal lines, and return to where you started). The two leftmost diagrams do not contain closed paths, and are referred to as tree level diagrams, while the two rightmost diagrams are referred to as loop level diagrams. Since tree level diagrams contain fewer vertices, they contribute more to the overall probability of an event.

Feynman diagrams are useful because each component of the diagram has a term associated with it, and the product of all the terms gives us the probability amplitude associated with that diagram. There are three types of components in a Feynman diagram: interaction vertices, which denote particle interactions; external lines, which are connected to only one interaction vertex and represent real particles coming in or real particles going out; and internal lines, which are connected to interaction vertices at both ends and represent what are called virtual particles.
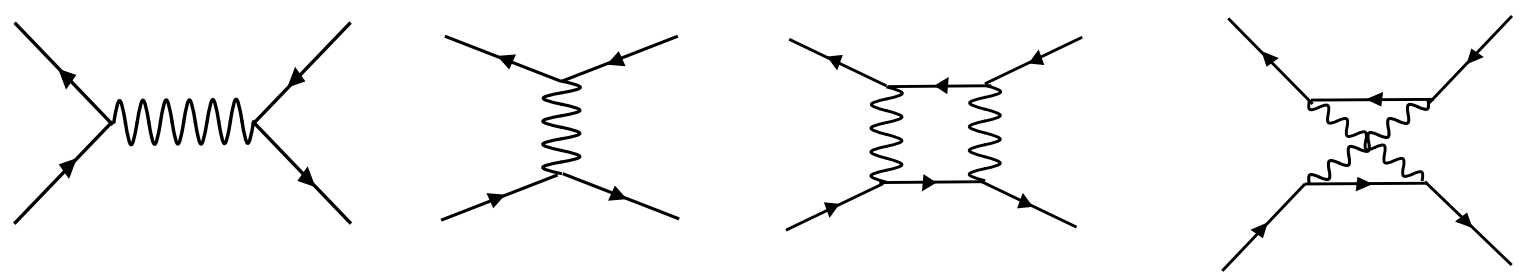

Figure 1: Feynman diagrams for electron-positron scattering. In each diagram, the direction of time is from left to right. Starting with the leftmost diagram, these diagrams represent: an electron and positron annihilating to produce a virtual photon, which then decays back into an electron and positron; an electron and positron approaching each other, exchanging a virtual photon, and moving away from each other; an electron and positron exchanging a virtual photon twice in a row before separating; and an electron and positron which each emit a virtual photon, subsequently absorb the photon emitted by the other, and move apart. 


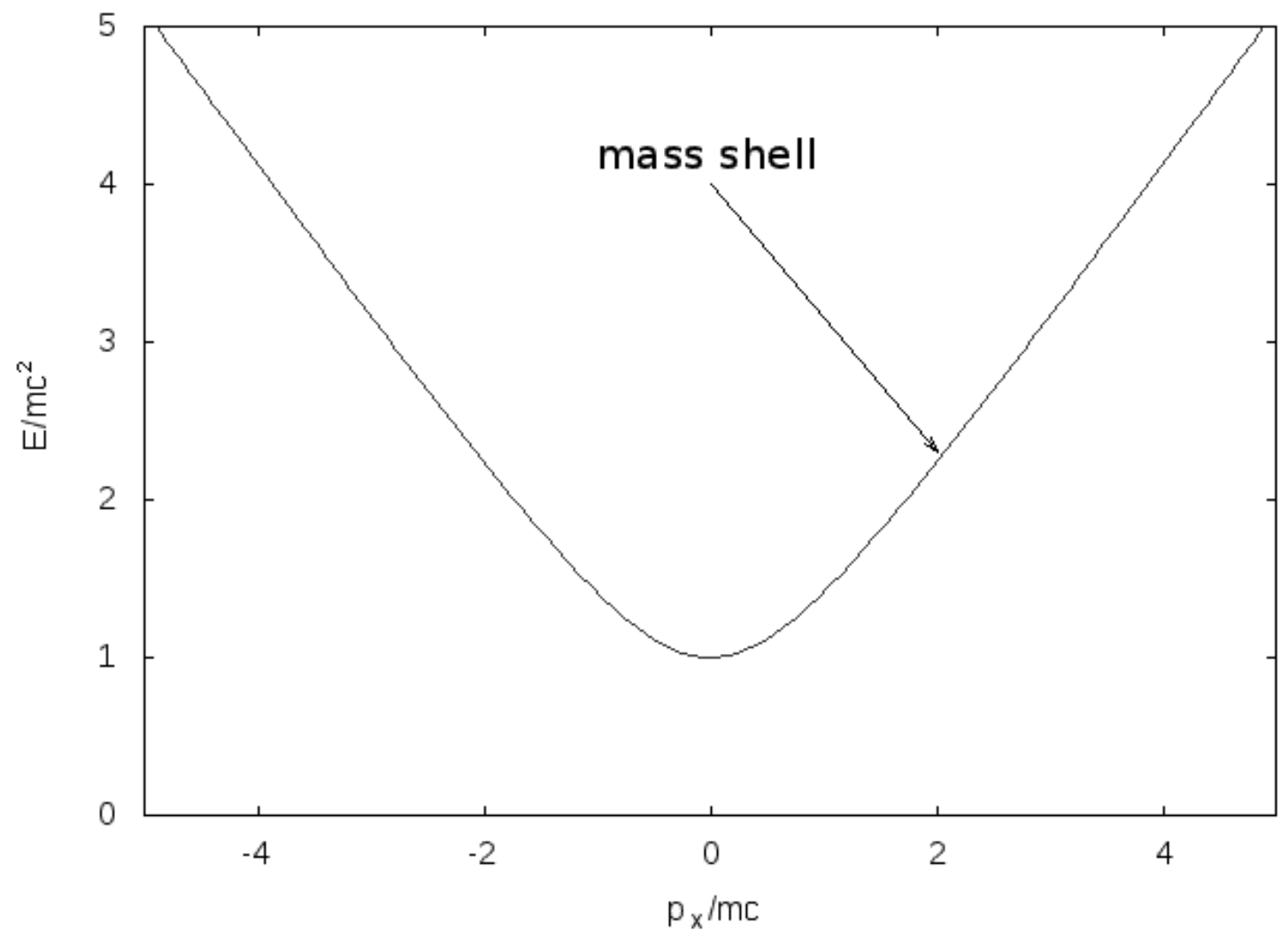

Figure 2: A graph of the mass shell for a particle of mass $m$ moving in one dimension. In general, real particles must have a combined momentum and energy which places them on the mass shell, while virtual particles are not so restricted.

External, real particles are forced to satisfy the relativistic relation

$$
E^{2}=\left(m c^{2}\right)^{2}+(p c)^{2},
$$

where $E$ is particle energy, $m$ is particle mass, and $p$ is particle momentum. If one chooses to graph the relationship between particle momentum and energy, as in Fig. 2, the result is a thin "mass shell", which real particles are forced to adhere to. However, virtual particles that are represented by internal lines are not restricted to the mass shell; their energy and momentum can have any value at all, so long as energy and momentum are conserved at interaction vertices in the Feynman diagram. For this reason, virtual particles are sometimes known as off-shell, while real particles are 


\section{known as on-shell.}

If a particle can interact with fields that give rise to particles lighter than it, said particle will generally decay directly into those lighter particles, which may undergo decays of their own. Sometimes, however, there will be a collection of lighter particles that the parent particle cannot decay into directly, but that the parent particle can decay into indirectly, by first decaying into an offshell version of a heavier particle. The offshell, intermediate particle lasts fleetingly, before itself decaying into much lighter products. In such cases, the initial decay to the heavier particle is often referred to as an off-shell decay.

When one is trying to find a new, heavy particle, it is usually not possible to observe the particle directly, since heavy particles tend to decay rapidly into lighter decay products. In that case, it is usually best to look for the decay products instead, and infer the new particle's properties from them. Unfortunately, in high-energy particle colliders, there are often so many particles being created in a single collision, and so many collisions happening in a given space of time, that it is simply not possible to look through all of the data, by hand or even with computers, in order to find any and all strange new decay products that don't fit into the Standard Model predictions. If one wishes to search for the decay products of a new particle, one must know what those new decay products are, so that one may tell the collision-analysis computers what sorts of decays they should be looking out for. As such, when searching for a new particle, it is not only necessary to know what its decay modes should be (i.e. what groups of particles it tends to decay into $\left.P \rightarrow p_{1}+p_{2}+\ldots\right)$, but also how likely each decay mode is. Since we will be talking about exactly these quantities in Section 3, we will introduce some terminology here: the rate at which a particle $P$ decays is called its total width $\Gamma_{P}$, the rate at which a particle decays by a specific decay mode is called the partial width of that decay mode $\Gamma\left(P \rightarrow p_{1}+p_{2}+\ldots\right)$, and the probability that a particle decays through a given decay mode is called that 
decay mode's branching ratio $B R\left(P \rightarrow p_{1}+p_{2}+\ldots\right)=\Gamma\left(P \rightarrow p_{1}+p_{2}+\ldots\right) / \Gamma_{P}$.

Now that we have discussed the terminology surrounding particle interaction and decay, we will move on to a discussion of some of the symmetries found in quantum particle fields, and how the existence of a Higgs boson is necessary in order to preserve these symmetries.

\subsection{Fermions and chiral symmetry}

In quantum field theory, the fundamental particles are produced by fields that permeate all of space, with each particle arising as a disturbance that propagates through these fields. A particle field $\phi(x)$, with Lagrangian density $\mathcal{L}\left(\phi, \partial_{\mu} \phi\right)$, is a special mathematical quantity known as an operator, and is quantized via the relations

$$
[\phi(\vec{x}, t), \Pi(\vec{y}, t)]_{ \pm}=i \delta(\vec{x}-\vec{y}), \quad[\phi(\vec{x}, t), \phi(\vec{y}, t)]_{ \pm}=[\Pi(\vec{x}, t), \Pi(\vec{y}, t)]_{ \pm}=0
$$

where $\Pi(x)=\partial \mathcal{L} / \partial \dot{\phi}(x)$ and using commutation relations $[a, b]_{-}=a b-b a$ for boson fields and anticommutation relations $[a, b]_{+}=a b+b a$ for fermion fields. Each field's behaviour is governed by different kinds of terms in the Lagrangian, depending on whether it is associated with spin-1/2 particles (spinor fields), spin-1 particles (vector fields), or spin-0 particles (scalar fields).

Spin-1/2 fermion fields, $\psi$, obey the Dirac equation, and have the following Lagrangian:

$$
\mathcal{L}=\bar{\psi}(i \not \partial-m) \psi, \quad \not \partial=\gamma^{\mu} \partial_{\mu}, \quad \bar{\psi}=\psi^{\dagger} \gamma^{0},
$$

where $\gamma^{\mu}$ are the Dirac gamma matrices (which are associated with spinor operations), $\psi^{\dagger}$ is the Hermitian conjugate of $\psi$, and $\mu=0,1,2,3$ runs over the dimensions of spacetime.

The field $\psi$ of any spin-1/2 fermion can be projected into left-handed and right- 
handed chiral components

$$
\psi=\psi_{L}+\psi_{R}, \quad \psi_{L}=\frac{1}{2}\left(1-\gamma^{5}\right) \psi, \quad \psi_{R}=\frac{1}{2}\left(1+\gamma^{5}\right) \psi, \quad \gamma^{5}=-i \gamma^{0} \gamma^{1} \gamma^{2} \gamma^{3}
$$

The Lagrangian can be expressed in terms of these components to get

$$
\mathcal{L}=i \bar{\psi}_{L} \not \partial \psi_{L}+i \bar{\psi}_{R} \not \partial \psi_{R}-m\left(\bar{\psi}_{L} \psi_{R}+\bar{\psi}_{R} \psi_{L}\right)
$$

One can see that, if $m=0$, then the components $\psi_{L}$ and $\psi_{R}$ can be transformed independently

$$
\psi_{L} \rightarrow e^{-i \theta_{L}} \psi_{L}, \quad \psi_{R} \rightarrow e^{-i \theta_{R}} \psi_{R}
$$

without changing the value of the Lagrangian. This invariance is known as "chiral symmetry". However, if $m \neq 0$, then the chiral components can no longer be transformed independently and the symmetry is broken. Because the weak interactions involve only the left-handed chiral projections of fermions, it is most natural to assume that the fundamental fermions in the Standard Model are massless, despite the experimental observation of their nonzero mass values. The resolution of this conflict (via the Higgs mechanism) will be discussed shortly, after we discuss a similar problem involving the massive gauge bosons.

\subsection{Gauge symmetry}

Let $\psi$ be a field, whether boson or fermion. If such a field undergoes a phase transformation of the form

$$
\psi(x) \rightarrow U(\alpha) \psi(x), \quad U(\alpha) \equiv \exp (i \alpha)
$$

then the Lagrangian of the field remains invariant, e.g. for fermions, 


$$
\mathcal{L} \rightarrow \overline{\left(e^{i \alpha} \psi\right)}(i \not \partial-m)\left(e^{i \alpha} \psi\right)=e^{-i \alpha} \bar{\psi}(i \not \partial-m) e^{i \alpha} \psi=\bar{\psi}(i \not \partial-m) \psi=\mathcal{L} .
$$

Since the transformation is independent of position $x$, the transformation is known as a "global transformation". However, if we try to use a local (or gauge) transformation,

$$
\psi(x) \rightarrow U(\alpha(x)) \psi(x)
$$

then the position dependence of the phase $\alpha(x)$ means that terms in the Lagrangian which contain partial derivatives $\partial_{\mu} \psi$ will no longer be invariant under the transformation. To resolve this problem, one needs to replace the partial derivative $\partial_{\mu}$ with a covariant derivative operator $D_{\mu}$, defined such that

$$
\psi(x) \rightarrow U(\alpha(x)) \psi(x), \quad D_{\mu} \psi(x) \rightarrow U(\alpha(x)) D_{\mu} \psi(x)
$$

Introducing a vector field, $A_{\mu}(x)$, allows one to define the covariant derivative as

$$
D_{\mu}=\partial_{\mu}+i f A_{\mu}
$$

where $f$ is a coupling constant. In order to satisfy our requirement (10) for $D_{\mu}, A_{\mu}$ must transform as

$$
A_{\mu} \rightarrow A_{\mu}+\frac{i}{f} \partial_{\mu} U(\alpha(x)) \cdot U^{-1}(\alpha(x))
$$

The Lagrangian contribution made by $A_{\mu}$ itself is also invariant under (12):

$$
\mathcal{L}_{A}=-\frac{1}{4} F_{\mu \nu} F^{\mu \nu}, \quad F_{\mu \nu}=\partial_{\mu} A_{\nu}-\partial_{\nu} A_{\mu}=-\frac{i}{f}\left[D_{\mu}, D_{\nu}\right]
$$

A gauge boson mass term, proportional to $A^{\mu} A_{\mu}$, would not be invariant under a gauge transformation and thus is not included in $\mathcal{L}_{A}$.

All vector fields arise from gauge symmetries. The most notable example of a 
gauge theory is electromagnetism, which arises from an abelian symmetry like the gauge symmetry outlined above. However, more complex, non-abelian symmetries also exist, and give rise to their own vector fields. However, regardless of whether the vector field is abelian or non-abelian, mass terms must be absent from their Lagrangians in order to preserve the associated gauge symmetry. Unfortunately, the weak gauge bosons, $W^{ \pm}$and $Z$, are massive particles, once again forcing us to deal with symmetry-breaking behaviour.

To demonstrate the mathematics of non-abelian gauge symmetries, let us suppose we have a set of $r$ fields $\Psi=\left\{\psi_{j}\right\}(j=1,2, \ldots, r)$, which form a representation of some non-abelian group $G$. Let us suppose that the Lie algebra of $G$ (that is, the set of elements $\mathbf{X}$ such that $\exp (-i \mathbf{X}) \in G)$ has $n$ generators $\mathbf{G}^{a}(a=1, \ldots, n)$. The structure constants $c^{a b c}(a, b, c=1, \ldots, n)$ of the Lie algebra are defined such that

$$
\left[\mathbf{G}^{a}, \mathbf{G}^{b}\right]=i c^{a b c} \mathbf{G}^{c}
$$

A gauge transformation on $\Psi$ will then be of the form

$$
\Psi \rightarrow \Psi^{\prime}=\mathbf{U}(\vec{\alpha}) \Psi, \quad \mathbf{U}(\vec{\alpha})=\exp \left(-i \alpha^{a}(x) \mathbf{G}^{a}\right) .
$$

Once again, we need a covariant derivative $\mathbf{D}_{\mu}$ such that

$$
\mathbf{D}_{\mu} \Psi \rightarrow\left(\mathbf{D}_{\mu} \Psi\right)^{\prime}=\mathbf{U}(\vec{\alpha})\left(\mathbf{D}_{\mu} \Psi\right)
$$

To achieve this, we introduce a new set of gauge fields $\vec{V}_{\mu}=\left\{V_{\mu}^{a}\right\}(a=1, \ldots, n)$ and define $\mathbf{D}_{\mu}$ as

$$
\mathbf{D}_{\mu}=\mathbf{I} \partial_{\mu}+i g \mathbf{V}_{\mu}, \quad \mathbf{V}_{\mu}=\mathbf{G}^{a} V_{\mu}^{a}
$$


with $g$ as the coupling constant for $\vec{V}_{\mu}$ and the gauge fields transforming as

$$
\mathbf{V}_{\mu} \rightarrow \mathbf{U}(\vec{\alpha}) \mathbf{V}_{\mu} \mathbf{U}^{-1}(\vec{\alpha})+\frac{i}{g} \partial_{\mu} \mathbf{U}(\vec{\alpha}) \cdot \mathbf{U}^{-1}(\vec{\alpha})
$$

Similarly to the abelian case, $\vec{V}_{\mu}$ makes a gauge-invariant contribution to the Lagrangian of the form

$$
\begin{gathered}
\mathcal{L}_{V}=-\frac{1}{2} \operatorname{Tr}\left(\mathbf{F}^{\mu \nu} \mathbf{F}_{\mu \nu}\right) \\
\mathbf{F}_{\mu \nu}=\partial_{\mu} \mathbf{V}_{\nu}-\partial_{\nu} \mathbf{V}_{\mu}+i g\left[\mathbf{V}_{\mu}, \mathbf{V}_{\nu}\right]=-\frac{i}{g}\left[\mathbf{D}_{\mu}, \mathbf{D}_{\nu}\right]
\end{gathered}
$$

If we choose our generators $\mathbf{G}^{a}$ such that

$$
\operatorname{Tr}\left(\mathbf{G}^{a} \mathbf{G}^{b}\right)=\frac{1}{2} \delta^{a b}
$$

then the Lagrangian contribution of the gauge fields $\vec{V}_{\mu}$ can be written as

$$
\begin{gathered}
\mathcal{L}_{V}=-\frac{1}{4} F_{a}^{\mu \nu} F_{\mu \nu}^{a}, \\
F_{\mu \nu}^{a}=\partial_{\mu} V_{\nu}^{a}-\partial_{\nu} V_{\mu}^{a}-g c^{a b c} V_{\mu}^{b} V_{\nu}^{c} .
\end{gathered}
$$

As in the abelian case, requiring gauge invariance forbids a mass term for $\vec{V}_{\mu}$.

\subsection{Electroweak interactions}

The observed electromagnetic and weak interactions arise out of a combined electroweak interaction. The electroweak interactions preserve two quantities: weak isospin and weak hypercharge. The nonabelian $S U(2)$ gauge fields $\vec{W}_{\mu}=\left(W_{\mu}^{1}, W_{\mu}^{2}, W_{\mu}^{3}\right)$ couple to the weak isospin, and the abelian gauge field $B_{\mu}$ couples to the weak hypercharge. The gauge contribution to the Lagrangian due to these fields is then

$$
\mathcal{L}_{G}=-\frac{1}{4} F_{i}^{\mu \nu} F_{\mu \nu}^{i}-\frac{1}{4} B^{\mu \nu} B_{\mu \nu}
$$




$$
\begin{gathered}
F_{\mu \nu}^{i}=\partial_{\mu} W_{\nu}^{i}-\partial_{\nu} W_{\mu}^{i}-g_{2} \epsilon^{i j k} W_{\mu}^{j} W_{\nu}^{k} \\
B_{\mu \nu}=\partial_{\mu} B_{\nu}-\partial_{\nu} B_{\mu} .
\end{gathered}
$$

Only the left-handed components of the fermion fields possess weak isospin. As a result of this, the left-handed fermion components are paired into weak isospin doublets, while the right-handed components are put into weak isospin singlets. Looking at the first generation of fermions, these doublets and singlets can be expressed as follows:

$$
\ell_{L}=\left(\begin{array}{c}
\nu_{e} \\
e
\end{array}\right)_{L}, \quad q_{L}=\left(\begin{array}{l}
u \\
d
\end{array}\right)_{L}, \quad e_{R}, \quad u_{R}, \quad d_{R}
$$

(Right-handed neutrinos may or may not exist; regardless, their influence here is negligible and can generally be ignored.) Letting $\Psi_{L}$ represent the isodoublets and $\psi_{R}$ represent the isosinglets, the fermionic contribution to the Lagrangian is

$$
\mathcal{L}_{F}=\sum_{\Psi_{L}} \bar{\Psi}_{L} i \not D \Psi_{L}+\sum_{\psi_{R}} \bar{\psi}_{R} i \not D \psi_{R}, \quad \not D=\mathbf{D}_{\mu} \gamma^{\mu}, \quad \not D=D_{\mu} \gamma^{\mu}
$$

The covariant derivative for the right-handed fields is

$$
D_{\mu} \psi_{R}=\left(\partial_{\mu}+i \frac{g_{1}}{2} Y_{\mathrm{w}} B_{\mu}\right) \psi_{R}
$$

while the covariant derivative for the left-handed fields is

$$
\mathbf{D}_{\mu} \Psi_{L}=\left(\mathbf{I}\left(\partial_{\mu}+i \frac{g_{1}}{2} Y_{\mathrm{w}} B_{\mu}\right)+i g_{2} \frac{\overrightarrow{\boldsymbol{\sigma}}}{2} \cdot \vec{W}_{\mu}\right) \Psi_{L}
$$

where $Y_{\mathrm{w}}$ is the hypercharge and $\overrightarrow{\boldsymbol{\sigma}}=\left(\sigma_{1}, \sigma_{2}, \sigma_{3}\right)$ are the Pauli matrices.

In the next section, we will see how the Higgs mechanism influences these gauge fields to produce the photon and the massive $W^{ \pm}$and $Z$ bosons. 


\subsection{Higgs mechanism}

Due to the symmetry considerations above, the Standard Model is only invariant under weak isospin if the fundamental particles (i.e. the leptons, quarks and gauge bosons) are considered massless. However, since many of the fundamental particles are observed to have mass, we have some difficulty reconciling theory with experiment. To deal with the problem, a set of complex scalar fields are introduced (see, e.g., p.55 of Ref. [2]):

$$
\boldsymbol{\Phi}=\left(\begin{array}{l}
\phi^{+} \\
\phi^{0}
\end{array}\right) .
$$

These fields make a gauge contribution $\mathcal{L}_{H G}$ and a fermion interaction contribution $\mathcal{L}_{H F}$ to the Lagrangian. The gauge contribution is

$$
\begin{aligned}
\mathcal{L}_{H G} & =\left(D^{\mu} \boldsymbol{\Phi}\right)^{\dagger} D_{\mu} \boldsymbol{\Phi}-V(\boldsymbol{\Phi}), \\
D_{\mu} \boldsymbol{\Phi} & =\left(\mathbf{I}\left(\partial_{\mu}+i \frac{g_{1}}{2} Y_{w} B_{\mu}\right)+i g_{2} \frac{\overrightarrow{\boldsymbol{\sigma}}}{2} \cdot \vec{W}_{\mu}\right) \boldsymbol{\Phi}, \\
V(\boldsymbol{\Phi}) & =-\mu^{2} \boldsymbol{\Phi}^{\dagger} \boldsymbol{\Phi}+\frac{\lambda}{4}\left(\boldsymbol{\Phi}^{\dagger} \boldsymbol{\Phi}\right)^{2} .
\end{aligned}
$$

If we consider only the first generation of fermions for simplicity, the Higgs-fermion interaction contribution is

$$
\begin{aligned}
& \mathcal{L}_{H F}=-f_{u} \bar{q}_{L} \tilde{\boldsymbol{\Phi}} u_{R}-f_{d} \bar{q}_{L} \boldsymbol{\Phi} d_{R}-f_{e} \bar{l}_{L} \boldsymbol{\Phi} e_{R}+\text { h.c. } \\
& q_{L}=\left(\begin{array}{l}
u \\
d
\end{array}\right)_{L}, \quad l_{L}=\left(\begin{array}{c}
\nu_{e} \\
e
\end{array}\right)_{L}, \quad \tilde{\boldsymbol{\Phi}}=i \boldsymbol{\sigma}_{2} \boldsymbol{\Phi}^{*}
\end{aligned}
$$

where $\boldsymbol{\Phi}^{*}$ is produced by taking the complex conjugates of the entries of $\boldsymbol{\Phi}$. Looking at the Higgs potential $V(\boldsymbol{\Phi})$, defined in (32), one sees that if $\mu$ and $\lambda$ are both positive, then the potential is minimized for the vacuum expectation value (VEV) of

$$
\left\langle\boldsymbol{\Phi}^{\dagger} \boldsymbol{\Phi}\right\rangle_{0}=\frac{v^{2}}{2}, \quad v \equiv \sqrt{\frac{4 \mu^{2}}{\lambda}}
$$


By inserting a Higgs field with the above VEV into the Lagrangian, one gets the Higgs contribution in the ground state. Then, combining the first two components of the isospin fields to get the complex field

$$
W_{\mu}^{ \pm}=\sqrt{\frac{1}{2}}\left(W_{\mu}^{1} \mp i W_{\mu}^{2}\right),
$$

we find the mass terms

$$
\begin{aligned}
\mathcal{L}_{\text {mass }}=-\frac{v}{\sqrt{2}} & \left(f_{u} \bar{u} u+f_{d} \bar{d} d+f_{e} \bar{e} e\right) \\
+ & \left(\frac{v g_{2}}{2}\right)^{2} W_{\mu}^{+} W^{-\mu} \\
& +\frac{v^{2}}{8}\left(W_{\mu}^{3}, B_{\mu}\right)\left(\begin{array}{cc}
g_{2}^{2} & -g_{1} g_{2} \\
-g_{1} g_{2} & g_{1}^{2}
\end{array}\right)\left(\begin{array}{c}
W^{3 \mu} \\
B^{\mu}
\end{array}\right) .
\end{aligned}
$$

The electroweak symmetry is thus spontaneously broken in the ground state, giving us the fermion masses

$$
m_{\alpha}=\frac{v}{\sqrt{2}} f_{\alpha} \quad(\alpha=u, d, e, \ldots)
$$

and the $W^{ \pm}$boson mass

$$
M_{W}=\frac{v}{2} g_{2}
$$

The mass matrix in the last line of (36) can be diagonalized by mixing the third isospin field with the hypercharge field to get the new fields

$$
Z_{\mu}=\cos \theta_{\mathrm{w}} W_{\mu}^{3}-\sin \theta_{\mathrm{w}} B_{\mu}, \quad A_{\mu}=\sin \theta_{\mathrm{w}} W_{\mu}^{3}+\cos \theta_{\mathrm{w}} B_{\mu},
$$

where $\theta_{\mathrm{w}}$ is known as the "weak mixing angle" and is defined by

$$
\tan \theta_{\mathrm{w}}=g_{1} / g_{2} .
$$


The field $Z_{\mu}$ is associated with the $Z$ boson, while $A_{\mu}$ is the electromagnetic field associated with the photon $\gamma$. The resulting masses are then

$$
M_{Z}=\frac{v}{2} \sqrt{g_{1}^{2}+g_{2}^{2}}, \quad M_{\gamma}=0 .
$$

At this point, there are a couple of facts worth mentioning. First, the photon has zero mass, as it should if all of electrodynamics is to remain valid. Next, the $W$ and $Z$ boson masses are related by

$$
\rho=\frac{M_{W}^{2}}{M_{Z}^{2} \cos ^{2} \theta_{\mathrm{w}}}=1 .
$$

Finally, one can see from the above that the fermion and gauge boson masses are all proportional to $v=246 \mathrm{GeV}$, known as the weak scale. This is the characteristic energy scale of the electroweak interactions.

With some additional work, the mass of the Higgs boson can also be derived. The complex Higgs fields can be represented by four real scalar fields, $H^{0}$ and $\vec{\chi}=$ $\left\{\chi_{i}\right\}(i=1,2,3)$ :

$$
\begin{gathered}
\mathbf{\Phi}=\mathbf{U}^{-1}(\vec{\chi})\left(\begin{array}{c}
0 \\
\left(v+H^{0}\right) / \sqrt{2}
\end{array}\right), \\
\mathbf{U}(\vec{\chi})=\exp (i \vec{\chi} \cdot \overrightarrow{\boldsymbol{\sigma}} / v) .
\end{gathered}
$$

One can then perform the gauge transformation

$$
\begin{gathered}
\Phi^{\prime}=\mathbf{U}(\vec{\chi}) \mathbf{\Phi}=\left(\begin{array}{c}
0 \\
\left(v+H^{0}\right) / \sqrt{2}
\end{array}\right), \\
\psi_{L}^{\prime}=\mathbf{U}(\vec{\chi}) \psi_{L}, \quad \psi_{R}^{\prime}=\psi_{R}, \quad B_{\mu}^{\prime}=B_{\mu}, \\
\frac{\overrightarrow{\boldsymbol{\sigma}}}{2} \cdot \vec{W}_{\mu}^{\prime}=\mathbf{U}(\vec{\chi}) \frac{\overrightarrow{\boldsymbol{\sigma}}}{2} \cdot \vec{W}_{\mu} \mathbf{U}^{-1}(\vec{\chi})+i g_{2}^{-1} \partial_{\mu} \mathbf{U}(\vec{\chi}) \cdot \mathbf{U}^{-1}(\vec{\chi}),
\end{gathered}
$$


where $\psi_{L}$ represents all the fermion weak isodoublets and $\psi_{R}$ represents all the fermion weak isosinglets. Under this transformation, the $\vec{\chi}$ fields get absorbed by the massive vector bosons and we are left with a single Higgs field $H^{0}$. The Higgs potential can then be expressed as

$$
V=-\frac{\mu^{2} v^{2}}{4}+\mu^{2} H_{0}^{2}+\frac{\lambda}{4} v H_{0}^{3}+\frac{\lambda}{16} H_{0}^{4}
$$

from which one can see that the Higgs mass is

$$
M_{H}=\sqrt{2} \mu=v \sqrt{\frac{\lambda}{2}},
$$

which is measured to be $125 \mathrm{GeV}$.

At present, the SM Higgs particle is the only scalar particle to have been discovered so far [3]. However, it remains possible that there exist additional scalar particles, produced by additional Higgs fields and making their own contributions to the SM particle masses. We will discuss one possible model which introduces additional scalar fields. 


\section{The Georgi-Machacek model, GMCALC, and the H5plane benchmark}

The GM model $[4,5]$ was first introduced in 1985, as a way of adding new scalar particles to the Standard Model through the use of triplet scalar fields. Previous to this, proposed models that contained scalar fields larger than a doublet tended to violate custodial symmetry. Since custodial symmetry is responsible for the fact that the rho parameter remains equal to one (see Eq.(42)), any model violating custodial symmetry is subject to stringent constraints to avoid contradicting experimental data. The GM model gets around this problem through the use of two scalar triplet fields, which combine in such a way as to prevent custodial symmetry breaking. The presence of the scalar triplet fields produces a number of additional scalar particles, including a doubly-charged Higgs boson, $H_{5}^{ \pm \pm}$(models that only contain scalar doublets cannot produce a doubly-charged Higgs).

In addition, the $H_{5}$ bosons couple to the weak gauge bosons but not to fermions, and the $W$ and $Z$ bosons could potentially receive a large portion of their mass from interaction with the new $H_{5}$ Higgs particles. Due to this fact, the GM model is being used by LHC experiments as a standard for interpreting searches for decays of charged Higgs particles to vector bosons, $H^{ \pm} \rightarrow W Z, H^{ \pm \pm} \rightarrow W^{ \pm} W^{ \pm}$. It is this interest from experimentalists which compelled us to study the GM model further, so as to provide predictions for its behaviour that experimentalists could utilize. In this section, we talk in detail about the GM model, the GMCALC program which we used to derive

\begin{tabular}{|c|ccccc|}
\hline & $\mathbf{- 2 e}$ & $\mathbf{- 1 e}$ & $\mathbf{0 e}$ & $+\mathbf{1 e}$ & $+\mathbf{2 e}$ \\
\hline $\mathbf{m}_{\mathbf{5}}$ & $H_{5}^{--}$ & $H_{5}^{-}$ & $H_{5}^{0}$ & $H_{5}^{+}$ & $H_{5}^{++}$ \\
$\mathbf{m}_{\mathbf{3}}$ & & $H_{3}^{-}$ & $H_{3}^{0}$ & $H_{5}^{+}$ & \\
$\mathbf{m}_{\mathbf{H}}$ & & & $H$ & & \\
$\mathbf{m}_{\mathbf{h}}$ & & & $h$ & & \\
\hline
\end{tabular}

Table 2: Higgs particles in the GM model, grouped by mass and charge. The $h$ particle is assumed to be the particle that was discovered at CERN in 2012. 
predictions for it, and the H5plane benchmark scenario which we used to effectively narrow down our search through the model's parameter space when predicting the possible range of its behaviours.

\subsection{The Georgi-Machacek model}

The scalar sector of the GM model consists of the usual complex doublet $\left(\phi^{+}, \phi^{0}\right)^{T}$ with hypercharge ${ }^{1} Y=1$, a real triplet $\left(\xi^{+}, \xi^{0},-\xi^{+*}\right)^{T}$ with $Y=0$, and a complex triplet $\left(\chi^{++}, \chi^{+}, \chi^{0}\right)^{T}$ with $Y=2$. The doublet is responsible for the fermion masses as in the SM. Custodial symmetry, required in order to avoid stringent constraints from the $\rho$ parameter (see Eq. (42)), is preserved at tree level (that is, for all particle interactions whose associated Feynman diagrams contain no loops) by imposing a global $\mathrm{SU}(2)_{L} \times \mathrm{SU}(2)_{R}$ symmetry on the scalar potential. To make this symmetry explicit, we write the doublet in the form of a bidoublet $\Phi$ and combine the triplets into a bitriplet $X$ :

$$
\Phi=\left(\begin{array}{cc}
\phi^{0 *} & \phi^{+} \\
-\phi^{+*} & \phi^{0}
\end{array}\right), \quad X=\left(\begin{array}{ccc}
\chi^{0 *} & \xi^{+} & \chi^{++} \\
-\chi^{+*} & \xi^{0} & \chi^{+} \\
\chi^{++*} & -\xi^{+*} & \chi^{0}
\end{array}\right)
$$

The VEVs are given by $\langle\Phi\rangle=\frac{v_{\phi}}{\sqrt{2}} I_{2 \times 2}$ and $\langle X\rangle=v_{\chi} I_{3 \times 3}$, where $I_{n \times n}$ is the $n \times n$ unit matrix and the $W$ and $Z$ boson masses constrain

$$
v_{\phi}^{2}+8 v_{\chi}^{2} \equiv v^{2}=\frac{1}{\sqrt{2} G_{F}} \approx(246 \mathrm{GeV})^{2} .
$$

The most general gauge-invariant scalar potential involving these fields that con-

\footnotetext{
${ }^{1}$ We use $Q=T^{3}+Y / 2$.
} 
serves custodial SU(2) is given, in the conventions of Ref. [6], by ${ }^{2}$

$$
\begin{aligned}
V(\Phi, X)= & \frac{\mu_{2}^{2}}{2} \operatorname{Tr}\left(\Phi^{\dagger} \Phi\right)+\frac{\mu_{3}^{2}}{2} \operatorname{Tr}\left(X^{\dagger} X\right)+\lambda_{1}\left[\operatorname{Tr}\left(\Phi^{\dagger} \Phi\right)\right]^{2}+\lambda_{2} \operatorname{Tr}\left(\Phi^{\dagger} \Phi\right) \operatorname{Tr}\left(X^{\dagger} X\right) \\
& +\lambda_{3} \operatorname{Tr}\left(X^{\dagger} X X^{\dagger} X\right)+\lambda_{4}\left[\operatorname{Tr}\left(X^{\dagger} X\right)\right]^{2}-\lambda_{5} \operatorname{Tr}\left(\Phi^{\dagger} \tau^{a} \Phi \tau^{b}\right) \operatorname{Tr}\left(X^{\dagger} t^{a} X t^{b}\right) \\
& -M_{1} \operatorname{Tr}\left(\Phi^{\dagger} \tau^{a} \Phi \tau^{b}\right)\left(U X U^{\dagger}\right)_{a b}-M_{2} \operatorname{Tr}\left(X^{\dagger} t^{a} X t^{b}\right)\left(U X U^{\dagger}\right)_{a b} .
\end{aligned}
$$

Here the $\mathrm{SU}(2)$ generators for the doublet representation are $\tau^{a}=\sigma^{a} / 2$ with $\sigma^{a}$ being the Pauli matrices, the generators for the triplet representation are

$$
t^{1}=\frac{1}{\sqrt{2}}\left(\begin{array}{ccc}
0 & 1 & 0 \\
1 & 0 & 1 \\
0 & 1 & 0
\end{array}\right), \quad t^{2}=\frac{1}{\sqrt{2}}\left(\begin{array}{ccc}
0 & -i & 0 \\
i & 0 & -i \\
0 & i & 0
\end{array}\right), \quad t^{3}=\left(\begin{array}{ccc}
1 & 0 & 0 \\
0 & 0 & 0 \\
0 & 0 & -1
\end{array}\right)
$$

and the matrix $U$, which rotates $X$ into the Cartesian basis, is given by [7]

$$
U=\left(\begin{array}{ccc}
-\frac{1}{\sqrt{2}} & 0 & \frac{1}{\sqrt{2}} \\
-\frac{i}{\sqrt{2}} & 0 & -\frac{i}{\sqrt{2}} \\
0 & 1 & 0
\end{array}\right)
$$

The physical fields can be organized by their transformation properties under the custodial SU(2) symmetry into a fiveplet, a triplet, and two singlets. The fiveplet and triplet states are given by

$$
\begin{aligned}
& H_{5}^{++}=\chi^{++}, \quad H_{5}^{+}=\frac{\left(\chi^{+}-\xi^{+}\right)}{\sqrt{2}}, \quad H_{5}^{0}=\sqrt{\frac{2}{3}} \xi^{0, r}-\sqrt{\frac{1}{3}} \chi^{0, r}, \\
& H_{3}^{+}=-s_{H} \phi^{+}+c_{H} \frac{\left(\chi^{+}+\xi^{+}\right)}{\sqrt{2}}, \quad H_{3}^{0}=-s_{H} \phi^{0, i}+c_{H} \chi^{0, i},
\end{aligned}
$$

\footnotetext{
${ }^{2} \mathrm{~A}$ translation table to other parameterizations in the literature has been given in the appendix
} of Ref. [6]. 
where the VEVs are parameterized by

$$
c_{H} \equiv \cos \theta_{H}=\frac{v_{\phi}}{v}, \quad s_{H} \equiv \sin \theta_{H}=\frac{2 \sqrt{2} v_{\chi}}{v}
$$

and we have decomposed the neutral fields into real and imaginary parts according to

$$
\phi^{0} \rightarrow \frac{v_{\phi}}{\sqrt{2}}+\frac{\phi^{0, r}+i \phi^{0, i}}{\sqrt{2}}, \quad \chi^{0} \rightarrow v_{\chi}+\frac{\chi^{0, r}+i \chi^{0, i}}{\sqrt{2}}, \quad \xi^{0} \rightarrow v_{\chi}+\xi^{0, r}
$$

The masses within each custodial multiplet are degenerate at tree level and can be written (after eliminating $\mu_{2}^{2}$ and $\mu_{3}^{2}$ in favor of the VEVs) $\operatorname{as}^{3}$

$$
\begin{aligned}
m_{5}^{2} & =\frac{M_{1}}{4 v_{\chi}} v_{\phi}^{2}+12 M_{2} v_{\chi}+\frac{3}{2} \lambda_{5} v_{\phi}^{2}+8 \lambda_{3} v_{\chi}^{2} \\
m_{3}^{2} & =\frac{M_{1}}{4 v_{\chi}}\left(v_{\phi}^{2}+8 v_{\chi}^{2}\right)+\frac{\lambda_{5}}{2}\left(v_{\phi}^{2}+8 v_{\chi}^{2}\right)=\left(\frac{M_{1}}{4 v_{\chi}}+\frac{\lambda_{5}}{2}\right) v^{2} .
\end{aligned}
$$

The two custodial-singlet mass eigenstates are given by

$$
h=\cos \alpha \phi^{0, r}-\sin \alpha H_{1}^{0 \prime}, \quad H=\sin \alpha \phi^{0, r}+\cos \alpha H_{1}^{0 \prime},
$$

where

$$
H_{1}^{0 \prime}=\sqrt{\frac{1}{3}} \xi^{0, r}+\sqrt{\frac{2}{3}} \chi^{0, r}
$$

and we will use the shorthand $c_{\alpha} \equiv \cos \alpha, s_{\alpha} \equiv \sin \alpha$. The mixing angle $\alpha$ and masses

\footnotetext{
${ }^{3}$ Note that the ratio $M_{1} / v_{\chi}$ can be written using the minimization condition $\partial V / \partial v_{\chi}=0$ as

$$
\frac{M_{1}}{v_{\chi}}=\frac{4}{v_{\phi}^{2}}\left[\mu_{3}^{2}+\left(2 \lambda_{2}-\lambda_{5}\right) v_{\phi}^{2}+4\left(\lambda_{3}+3 \lambda_{4}\right) v_{\chi}^{2}-6 M_{2} v_{\chi}\right],
$$

which is finite in the limit $v_{\chi} \rightarrow 0$. 
are given by

$$
\begin{aligned}
& \sin 2 \alpha=\frac{2 \mathcal{M}_{12}^{2}}{m_{H}^{2}-m_{h}^{2}}, \quad \cos 2 \alpha=\frac{\mathcal{M}_{22}^{2}-\mathcal{M}_{11}^{2}}{m_{H}^{2}-m_{h}^{2}} \\
& m_{h, H}^{2}=\frac{1}{2}\left[\mathcal{M}_{11}^{2}+\mathcal{M}_{22}^{2} \mp \sqrt{\left(\mathcal{M}_{11}^{2}-\mathcal{M}_{22}^{2}\right)^{2}+4\left(\mathcal{M}_{12}^{2}\right)^{2}}\right]
\end{aligned}
$$

where we choose $m_{h}<m_{H}$ (i.e. the $\mp$ in Eq. (62) is - for $m_{h}$ and + for $m_{H}$ ), and

$$
\begin{aligned}
\mathcal{M}_{11}^{2} & =8 \lambda_{1} v_{\phi}^{2}, \\
\mathcal{M}_{12}^{2} & =\frac{\sqrt{3}}{2} v_{\phi}\left[-M_{1}+4\left(2 \lambda_{2}-\lambda_{5}\right) v_{\chi}\right], \\
\mathcal{M}_{22}^{2} & =\frac{M_{1} v_{\phi}^{2}}{4 v_{\chi}}-6 M_{2} v_{\chi}+8\left(\lambda_{3}+3 \lambda_{4}\right) v_{\chi}^{2} .
\end{aligned}
$$

\subsection{GMCALC}

Numerical studies of the GM model are facilitated by the public code GMCALC (written in FORTRAN 77 and developed at Carleton University [8]), which takes a set of input parameters for the GM model and computes the mass spectrum, scalar sector mixing angles, and tree-level scalar couplings. It checks for adherence to theoretical constraints, i.e. checking the perturbative unitarity of the quartic scalar couplings, determining whether the scalar potential is bounded from below, and checking for the absence of any deeper minima which would break custodial symmetry. Experimental constraints from the $S$ parameter, $b \rightarrow s \gamma$, and $B_{s}^{0} \rightarrow \mu^{+} \mu^{-}$are also checked. Finally, the GMCALC code computes the branching ratios and total decay widths of all the scalars. For example, if one wishes to compute the branching ratio for the $H_{i} \rightarrow V_{1}^{*} V_{2}^{*}$ 
offshell decay, the GMCALC code does this by numerically evaluating the integral

$$
\begin{array}{rl}
\Gamma\left(H_{i} \rightarrow V_{1}^{*} V_{2}^{*}\right)=\frac{1}{\pi^{2}} \int_{0}^{m_{H_{i}}^{2}} & d Q_{1}^{2} \int_{0}^{\left(m_{H_{i}}-Q_{1}\right)^{2}} d Q_{2}^{2} \\
\times & \frac{Q_{1}^{2} \Gamma_{V_{1}} / M_{V_{1}}}{\left(Q_{1}^{2}-M_{V_{1}}^{2}\right)^{2}+M_{V_{1}}^{2} \Gamma_{V_{1}}^{2}} \\
& \times \frac{Q_{2}^{2} \Gamma_{V_{2}} / M_{V_{2}}}{\left(Q_{2}^{2}-M_{V_{2}}^{2}\right)^{2}+M_{V_{2}}^{2} \Gamma_{V_{2}}^{2}} \Gamma^{H_{i} V_{1} V_{2}}\left(Q_{1}^{2}, Q_{2}^{2}\right),
\end{array}
$$

where $\Gamma_{V_{i}}$ is the measured total width of gauge boson $V_{i}, Q_{i}^{2}$ is the square of the four-momentum of $V_{i}$, and $\Gamma^{H_{i} V_{1} V_{2}}\left(Q_{1}^{2}, Q_{2}^{2}\right)$ is the on-shell decay width for $H_{i} \rightarrow V_{1} V_{2}$ with the squared-masses of the gauge bosons $V_{1}$ and $V_{2}$ replaced by $Q_{1}^{2}$ and $Q_{2}^{2}$. The formula for $\Gamma^{H_{i} V_{1} V_{2}}\left(Q_{1}^{2}, Q_{2}^{2}\right)$ is

$$
\Gamma^{H_{i} V_{1} V_{2}}\left(Q_{1}^{2}, Q_{2}^{2}\right)=S_{V} \frac{\left|g_{H_{i} V_{1} V_{2}}\right|^{2} m_{H_{i}}^{3}}{64 \pi Q_{1}^{2} Q_{2}^{2}}\left[1-2 k_{1}-2 k_{2}+10 k_{1} k_{2}+k_{1}^{2}+k_{2}^{2}\right] \sqrt{\lambda\left(k_{1}, k_{2}\right)},
$$

where $S_{V}$ is a symmetry factor which is 1 if $V_{1}$ and $V_{2}$ are distinct bosons and is $1 / 2$ if $V_{1}$ and $V_{2}$ are identical, $g_{H_{i} V_{1} V_{2}}$ is the coupling constant between the scalar $H_{i}$ and the massive vector bosons $V_{1}$ and $V_{2}$, and $k_{1}=Q_{1}^{2} / m_{H_{i}}^{2}$ and $k_{2}=Q_{2}^{2} / m_{H_{i}}^{2}$. The function $\lambda$ is given by

$$
\lambda(x, y)=(1-x-y)^{2}-4 x y .
$$

Formulas such as these are utilized in the calculation of all relevant quantities. The input parameters for the GMCALC code are chosen to fix the values of the coefficients in Eq. (52); the values of these coefficients can be set directly, but other choices for the set of inputs are also available, depending on which parameters (e.g. scalar masses, mixing angles) one wishes to focus on.

We have used GMCALC 1.2.1 to compute decay rates, coupling constants, and mass differences for every scalar particle present in the GM model. To capture the range of possible behaviours for the model, we utilized the H5plane benchmark (discussed in the following subsection), and have recorded our results in Section 3. 


\subsection{H5plane benchmark}

In computational testing of a model, it is often prohibitively difficult to test the behaviour of the model over the full range of all possible values of input parameters. In such as case, a carefully chosen benchmark can be used, whose purpose is to provide a much smaller subset of the range of possible input values, but nevertheless captures nearly the full range of behaviours present in the model. Such a benchmark is indeed necessary for the GM model, whose behaviour is determined by seven free parameters (the scalar potential in Eq. (52) contains nine undetermined coefficients, two of which are fixed by the experimentally measured values of $v$ and $m_{h}$ ). Were we to attempt a full scan of the parameter space, computing across 100 possible values for each parameter, we would have $10^{14}$ points to search through! Utilizing the H5plane benchmark reduces the number of free parameters down to two, enabling us to study a much more manageable 10,000 points per parameter scan. We describe below how the H5plane benchmark is able to accomplish this.

The H5plane benchmark scenario for the GM model was introduced in Ref. [9]. It is designed to facilitate LHC searches for $H_{5}^{ \pm \pm}$and $H_{5}^{ \pm}$in vector boson fusion (VBF, in which two colliding fermions exchange a virtual weak gauge boson, which emits a Higgs particle), with decays to $W^{ \pm} W^{ \pm}$and $W^{ \pm} Z$, respectively. It is specified as in Table 3, in a form that is easily implemented in the model calculator GMCALC [8]. After imposing the existing direct search constraints on $H_{5}^{ \pm \pm}$, the benchmark has the following features:

- It comes close to fully populating the theoretically-allowed region of the $m_{5}-s_{H}$ plane for $m_{5} \in[200,3000] \mathrm{GeV}$, as shown in Fig. 3 (see below).

- It has $m_{3}>m_{5}$ over the whole benchmark plane, so that the Higgs-to-Higgs decays $H_{5} \rightarrow H_{3} H_{3}$ and $H_{5} \rightarrow H_{3} V$ are kinematically forbidden, leaving only the decays $H_{5} \rightarrow V V$ at tree level; i.e., $\operatorname{BR}\left(H_{5} \rightarrow V V\right)=1$. 


\begin{tabular}{lll}
\hline \hline Fixed parameters & Variable parameters & Dependent parameters \\
\hline$G_{F}=1.1663787 \times 10^{-5} \mathrm{GeV}^{-2}$ & $m_{5} \in[200,3000] \mathrm{GeV}$ & $\lambda_{2}=0.4\left(m_{5} / 1000 \mathrm{GeV}\right)$ \\
$m_{h}=125 \mathrm{GeV}$ & $s_{H} \in(0,1)$ & $M_{1}=\sqrt{2} s_{H}\left(m_{5}^{2}+v^{2}\right) / v$ \\
$\lambda_{3}=-0.1$ & & $M_{2}=M_{1} / 6$ \\
$\lambda_{4}=0.2$ & \\
\hline \hline
\end{tabular}

Table 3: Specification of the H5plane benchmark scenario for the Georgi-Machacek model. These input parameters correspond to INPUTSET $=4$ in GMCALC [8].

All other coefficients in the model are determined from the above parameters.

- The entire benchmark satisfies indirect constraints from $B$ physics, the most stringent of which is $b \rightarrow s \gamma[10]$.

- The region still allowed by direct searches is currently unconstrained by LHC measurements of the couplings of the $125 \mathrm{GeV}$ Higgs boson, as we show in this thesis.

In INPUTSET $=4$ of GMCALC, the nine parameters of the scalar potential in Eq. (52) are determined by the nine input parameters $m_{h}, m_{5}, s_{H}, \lambda_{2}, \lambda_{3}, \lambda_{4}, M_{1}, M_{2}$, and $v=\left(\sqrt{2} G_{F}\right)^{-1 / 2}$. The quartic coupling $\lambda_{5}$, expressed as a function of the above parameters, is written as

$$
\lambda_{5}=\frac{2 m_{5}^{2}}{3 c_{H}^{2} v^{2}}-\frac{\sqrt{2} M_{1}}{3 s_{H} v}-\frac{2 \sqrt{2} M_{2} s_{H}}{c_{H}^{2} v}-\frac{2 \lambda_{3} s_{H}^{2}}{3 c_{H}^{2}} .
$$

Once we know the value of $\lambda_{5}$, the quartic coupling $\lambda_{1}$ can be written as

$$
\lambda_{1}=\frac{1}{8 c_{H}^{2} v^{2}}\left\{m_{h}^{2}+\frac{3 c_{H}^{2} v^{2}\left[-M_{1}+\sqrt{2}\left(2 \lambda_{2}-\lambda_{5}\right) s_{H} v\right]^{2}}{2 \sqrt{2} M_{1} \frac{c_{H}^{2}}{s_{H}} v-6 \sqrt{2} M_{2} s_{H} v+4\left(\lambda_{3}+3 \lambda_{4}\right) s_{H}^{2} v^{2}-4 m_{h}^{2}}\right\} .
$$

The mass-squared parameter $\mu_{2}^{2}$ can be written as

$$
\mu_{2}^{2}=-4 \lambda_{1} c_{H}^{2} v^{2}-\frac{3}{8}\left(2 \lambda_{2}-\lambda_{5}\right) s_{H}^{2} v^{2}+\frac{3 \sqrt{2}}{8} M_{1} s_{H} v,
$$


and $\mu_{3}^{2}$ can be written as

$$
\mu_{3}^{2}=\frac{2}{3} m_{5}^{2}+\frac{\sqrt{2} M_{1} c_{H}^{2} v}{6 s_{H}}-2 \lambda_{2} c_{H}^{2} v^{2}-\frac{1}{6}\left(7 \lambda_{3}+9 \lambda_{4}\right) s_{H}^{2} v^{2}-\frac{\sqrt{2}}{2} M_{2} s_{H} v .
$$

These formulas can thus be used to take the 9 input parameters from INPUTSET $=4$ and compute every other coefficient in the GM model.

\subsection{Constraints on the H5plane benchmark}

In Fig. 3 we show the allowed region in the $m_{5}-s_{H}$ plane from a numerical scan over the full GM model (red points) and the allowed region for the H5plane benchmark scenario (entire region below both the black and blue curves), as generated using GMCALC 1.2.1 with $m_{h}=125 \mathrm{GeV}$. In both cases we impose the theoretical constraints from perturbative unitarity of the scalar quartic couplings, boundednessfrom-below of the scalar potential, and the absence of deeper alternative minima, as described in Ref. [6], as well as the indirect constraints from $b \rightarrow s \gamma$ and the $S$ parameter following Ref. [10] (we use the "loose" constraint on $b \rightarrow s \gamma$ as described in Ref. [10]); all of these constraints are implemented in GMCALC. We also impose the direct experimental constraint from a search for $H_{5}^{ \pm \pm}$by the CMS detector at CERN [11] (described in more detail below), which excludes the area above the blue curve in the context of the H5plane benchmark. Because the input parameters are subject to a lot of interdependent constraints, the ranges over which the input parameters would be uniformly distributed had to be evaluated successively, with the allowed range for subsequent parameters being constrained by the randomly determined values of previous parameters; the points were then checked to make sure that they satisfied theoretical and experimental requirements, to filter out any remaining spurious points. The red points represent the results of this scan over the full GM

model parameter space. The entire area below the black curve (obtained by scanning 


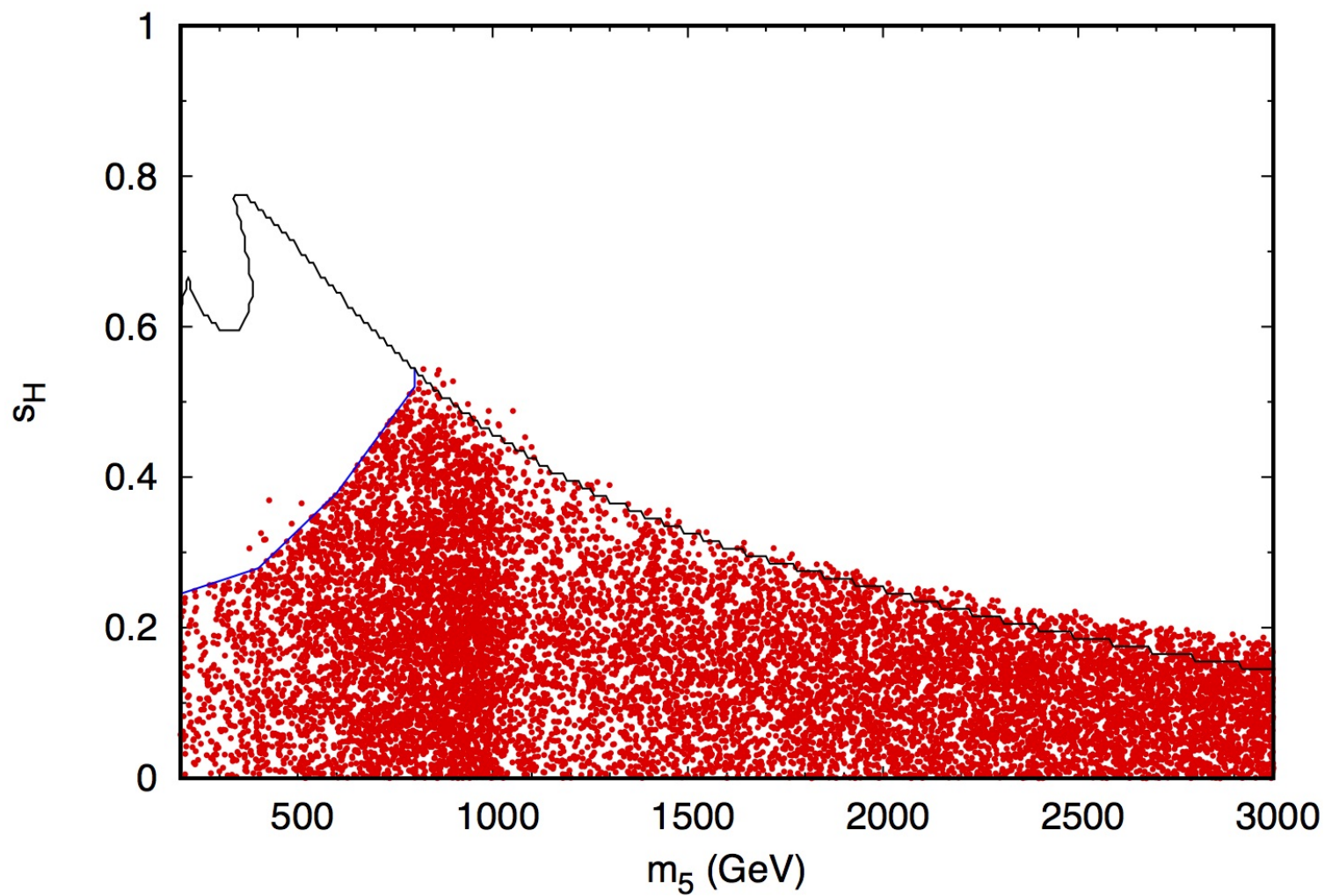

Figure 3: Theoretically and experimentally allowed parameter region in the $m_{5}-s_{H}$ plane in the H5plane benchmark (entire region below both the black and blue curves) and the full GM model (red points). The black curve delimits the region allowed by theoretical constraints in the H5plane benchmark and the blue curve represents the upper bound on $s_{H}$ from a direct search for $H_{5}^{ \pm \pm}$from Ref. [11]. See text for details.

over $m_{5}$ and $s_{H}$ in the H5plane benchmark) represents the theoretically-allowed region in the H5plane benchmark: as advertised, it nearly, but not quite entirely, populates the entire range of $s_{H}$ that is accessible in the full GM model for any given value of $m_{5}$ between 200 and $3000 \mathrm{GeV}$. This makes the H5plane scenario a good benchmark for the interpretation of searches for $H_{5}^{ \pm}$and $H_{5}^{ \pm \pm}$in vector boson fusion, for which the signal rate and kinematics depend only on $m_{5}, s_{H}$, and the $H_{5}$ branching ratios into vector boson pairs. We note, however, that the accessible ranges of other observables are not necessarily fully populated by the H5plane benchmark; this will be particularly dramatic for the mass splittings among the heavy Higgs bosons, as we discuss in Secs. 3.2 and 3.5 . 
The CMS search in Ref. [11] currently provides the most stringent direct experimental constraint on the GM model for $m_{5}$ above $200 \mathrm{GeV} .{ }^{4}$ This search looked for a doubly-charged scalar produced in vector boson fusion and decaying to two like-sign $W$ bosons that in turn decay leptonically, using $19.4 \mathrm{fb}^{-1}$ of proton-proton collision data at a centre-of-mass energy of $8 \mathrm{TeV}$. This search set a 95\% confidence level upper bound on the cross section times branching ratio, $\sigma\left(\mathrm{VBF} \rightarrow H^{ \pm \pm}\right) \times \mathrm{BR}\left(H^{ \pm \pm} \rightarrow\right.$ $\left.W^{ \pm} W^{ \pm}\right)$, as a function of the doubly-charged Higgs boson mass. The H5plane benchmark is designed so that $\operatorname{BR}\left(H_{5}^{ \pm \pm} \rightarrow W^{ \pm} W^{ \pm}\right)=1$, so the CMS constraint becomes an upper bound on the cross section $\sigma\left(\mathrm{VBF} \rightarrow H_{5}^{ \pm \pm}\right)$, which is proportional to $s_{H}^{2}$. We translated this into an upper bound on $s_{H}$ in the H5plane benchmark using the $\mathrm{VBF} \rightarrow H_{5}^{ \pm \pm}$cross sections calculated for the $8 \mathrm{TeV}$ LHC at next-to-next-to-leading order in QCD in Ref. [16] (we did not take into account the theoretical uncertainties in these predictions in computing the limit). This constraint in the H5plane benchmark is shown as the blue curve in Fig. 3; when combined with the theoretical constraints, it limits $s_{H}<0.55$ in the H5plane benchmark. In a full scan of the GM model, some allowed points appear that have $\operatorname{BR}\left(H_{5}^{ \pm \pm} \rightarrow W^{ \pm} W^{ \pm}\right)<1$, because decays into $H_{3}^{ \pm} W^{ \pm}$are kinematically allowed. Since the CMS constraint applies to the product $\sigma\left(\mathrm{VBF} \rightarrow H_{5}^{ \pm \pm}\right) \times \mathrm{BR}\left(H_{5}^{ \pm \pm} \rightarrow W^{ \pm} W^{ \pm}\right)$, this results in a few of the allowed red points in Fig. 3 falling above the blue curve. The number of such points is quite small, though, because most points in the full GM model scan that have $\operatorname{BR}\left(H_{5}^{ \pm \pm} \rightarrow W^{ \pm} W^{ \pm}\right)<1$ also have small $s_{H}$, putting them below the blue curve anyway.

Now that we have described the GM model, and the benchmark scenario under consideration, we will go over our results. We have computed decay rates, couplings, branching ratios, and mass splittings for the $H_{5}$ scalars, the $H_{3}$ scalars, and the $H$

\footnotetext{
${ }^{4}$ For comparison, the $95 \%$ confidence level constraint obtained in Ref. [12] from an ATLAS measurement of the cross section for like-sign $W$ boson pairs in vector boson fusion [13] excludes $s_{H}$ values above 0.39 for $m_{5}=200 \mathrm{GeV}$, rising to 0.74 for $m_{5}=600 \mathrm{GeV}$. LHC searches for $H_{5}^{ \pm}$in the $W Z$ final state $[14,15]$ are currently slightly less constraining than the search for $H_{5}^{ \pm \pm}$.
} 
and $h$ scalars, all of which we discuss in the next section. 


\section{Properties of the H5plane benchmark}

Using version 1.2.1 of the GMCALC program, we have computed a number of predictions for behaviour of the scalar sector of the GM model, to be used by experimentalists; we will discuss those predictions here. Focusing on scalar behaviour in the H5plane benchmark, with a few comparisons to the range of behaviours predicted for a full scan of the GM model parameter space, we will examine: the decay modes of the $H_{5}$ particles; the mass splitting between $H_{3}$ and $H_{5}$; the couplings and decay rates of the $h$ and $H$; the mass splitting between $H_{5}$ and $H$; and the decays of the $H_{3}$ particles.

\subsection{Decays of $H_{5}$}

The $H_{5}$ particles are produced via vector boson fusion (VBF, where two fermions collide and exchange a virtual weak gauge boson, which emits a Higgs), with a cross section that is proportional to $s_{H}^{2}$. One can utilize this fact, together with Tables 140143 from Ref. [9], to predict the cross sections of the $H_{5}$ particles at the $\sqrt{s}=13 \mathrm{TeV}$ LHC for any point in the H5plane benchmark (e.g. if $s_{H}=0.3$ and $m_{5}=1000 \mathrm{GeV}$, then the cross-sections at the LHC for VBF production of $H_{5}^{0}, H_{5}^{+}, H_{5}^{-}, H_{5}^{++}$, and $H_{5}^{--}$are $4.8 \mathrm{fb}, 7.0 \mathrm{fb}, 3.5 \mathrm{fb}, 11.2 \mathrm{fb}$, and $2.8 \mathrm{fb}$, respectively).

The H5plane benchmark was designed so that $m_{3}>m_{5}$ over the entire benchmark plane, so that the decay $H_{5}^{ \pm \pm} \rightarrow W^{ \pm} W^{ \pm}$is the only kinematically-allowed decay for $H_{5}^{ \pm \pm}$. This makes direct searches for $H_{5}^{ \pm \pm}$in the $W W$ final state particularly easy to interpret. Decays of $H_{5}^{ \pm}$to $W^{ \pm} Z$ are then also the only kinematically-accessible tree-level decay of $H_{5}^{ \pm}$, so that direct searches for the singly-charged state in this final state are also easy to interpret (the decay $H_{5}^{ \pm} \rightarrow W^{ \pm} \gamma$ is also allowed, but the associated Feynman diagrams must contain a loop, so it has a very small branching ratio for $m_{5} \geq 200 \mathrm{GeV}$ ). This fact was used in the GM model interpretation of the ATLAS and CMS searches for $H_{5}^{ \pm}$in Refs. $[14,15]$ (these searches are less constraining 


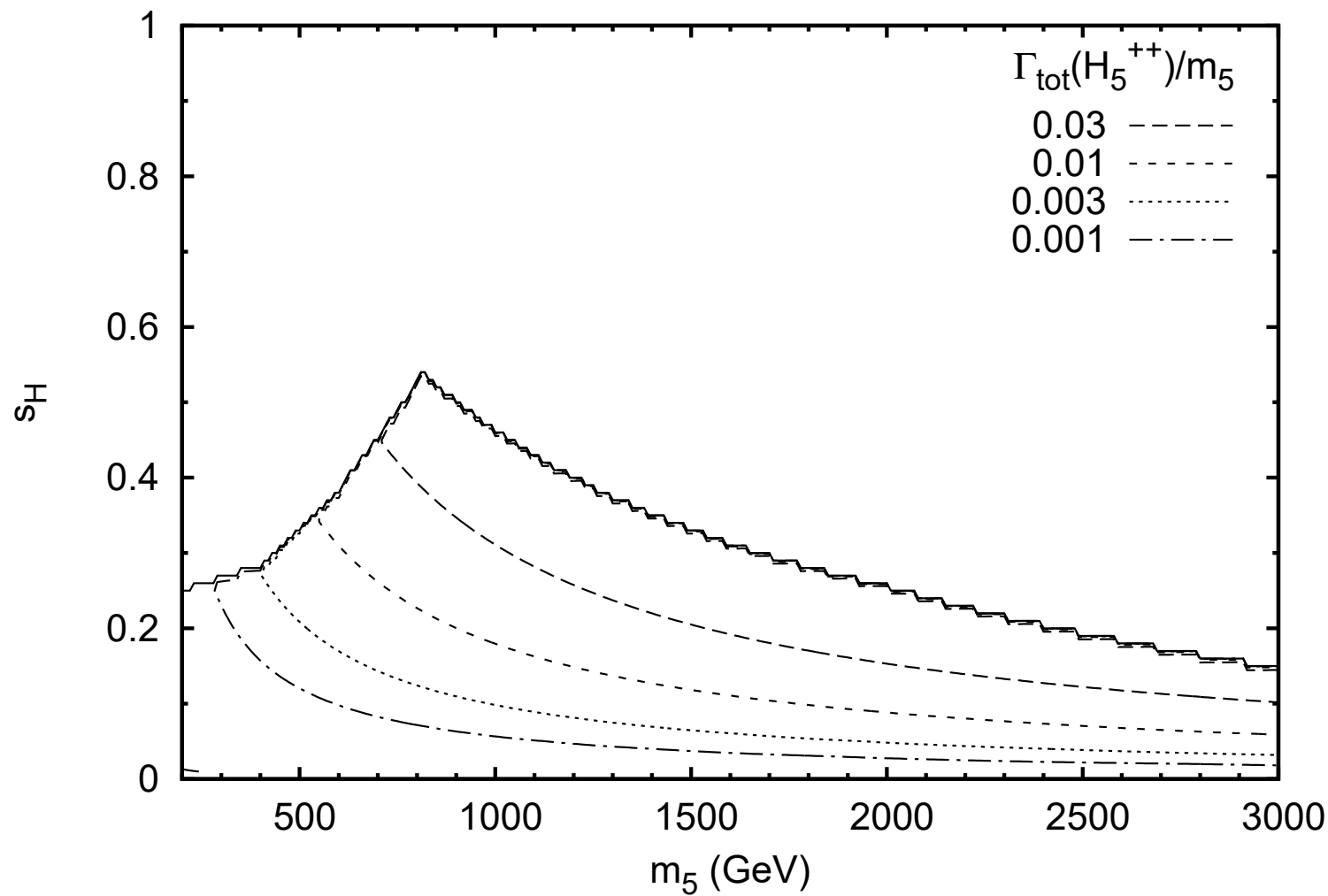

Figure 4: Contours of $\Gamma_{\text {tot }} / m_{5}$ for $H_{5}^{++}$in the GM model H5plane benchmark. The value of $\Gamma_{\text {tot }} / m_{5}$ reaches a maximum of 0.08 along the upper boundary of the allowed region for $m_{5} \gtrsim 800 \mathrm{GeV}$, and goes to zero at $s_{H}=0$.

on the GM model parameter space than that of Ref. [11]).

In Fig. 4 we show the total width of $H_{5}^{ \pm \pm}$normalized to its mass. This widthto-mass ratio reaches a maximum of $8 \%$ for the largest theoretically-allowed values of $s_{H}$ when $m_{5}>800 \mathrm{GeV}$. Fig. 5 shows the deviation from unity of the ratio of partial widths of $H_{5}^{ \pm}$and $H_{5}^{0}$ divided by that of $H_{5}^{ \pm \pm}$as a function of $m_{5}$. These ratios are independent of $s_{H}$. The widths of $H_{5}^{ \pm}$and $H_{5}^{0}$ are about $10 \%$ smaller than that of $H_{5}^{ \pm \pm}$for $m_{5} \sim 200 \mathrm{GeV}$, with the difference decreasing to less than $1 \%$ for $m_{5} \gtrsim 1000 \mathrm{GeV}$. In the H5plane benchmark, this width difference is solely due to the kinematic effect of the different masses of the $W W, W Z$, and $Z Z$ final states. 


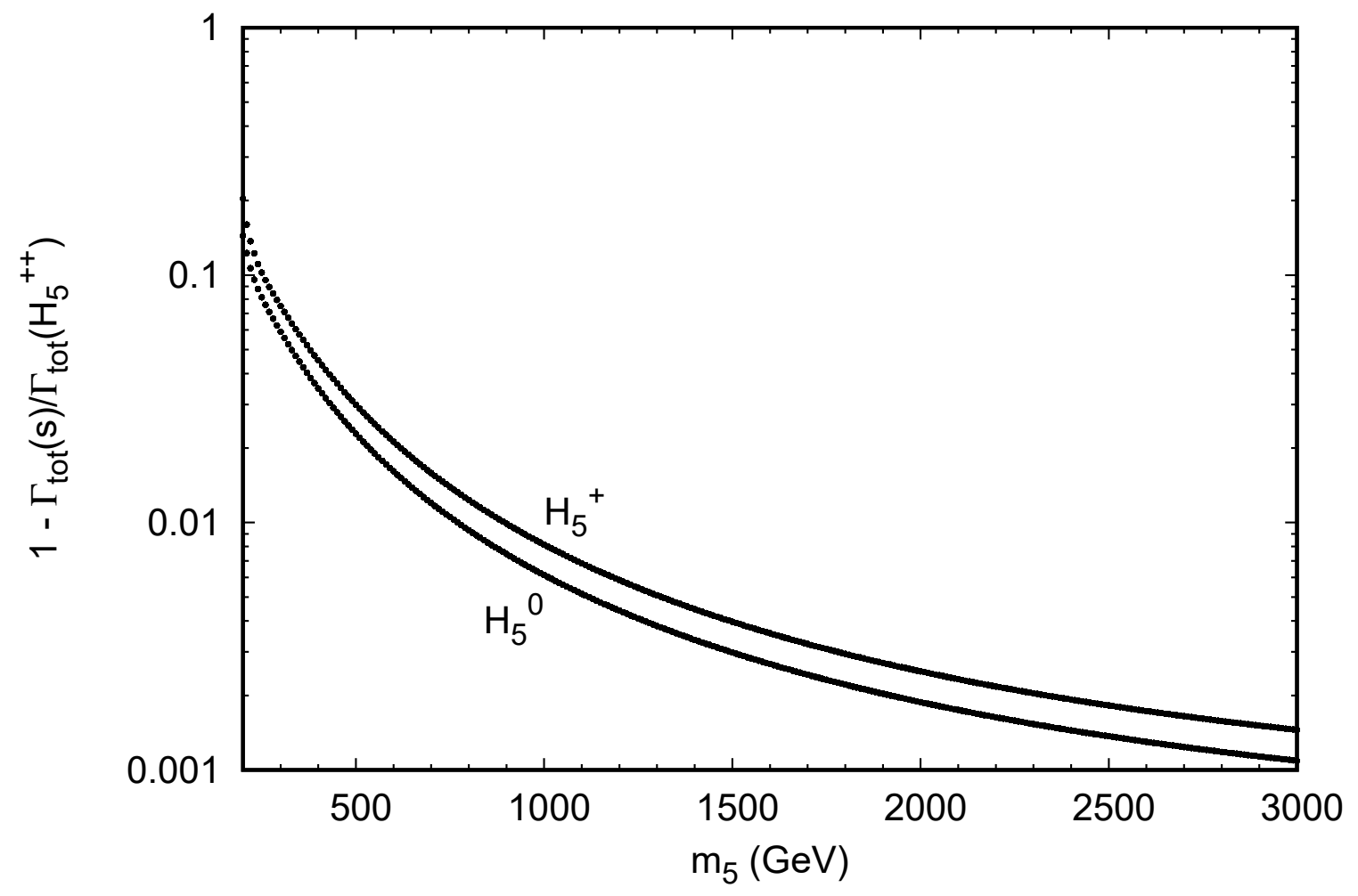

Figure 5: Deviation from unity of the ratio of total widths of scalar $s=H_{5}^{+}$and $H_{5}^{0}$ to that of $H_{5}^{++}$as a function of $m_{5}$ in the H5plane benchmark (these ratios are independent of $s_{H}$ ). Direct constraints from a CMS search for $H_{5}^{ \pm \pm} \rightarrow W^{ \pm} W^{ \pm}$in vector boson fusion [11] have been applied. 


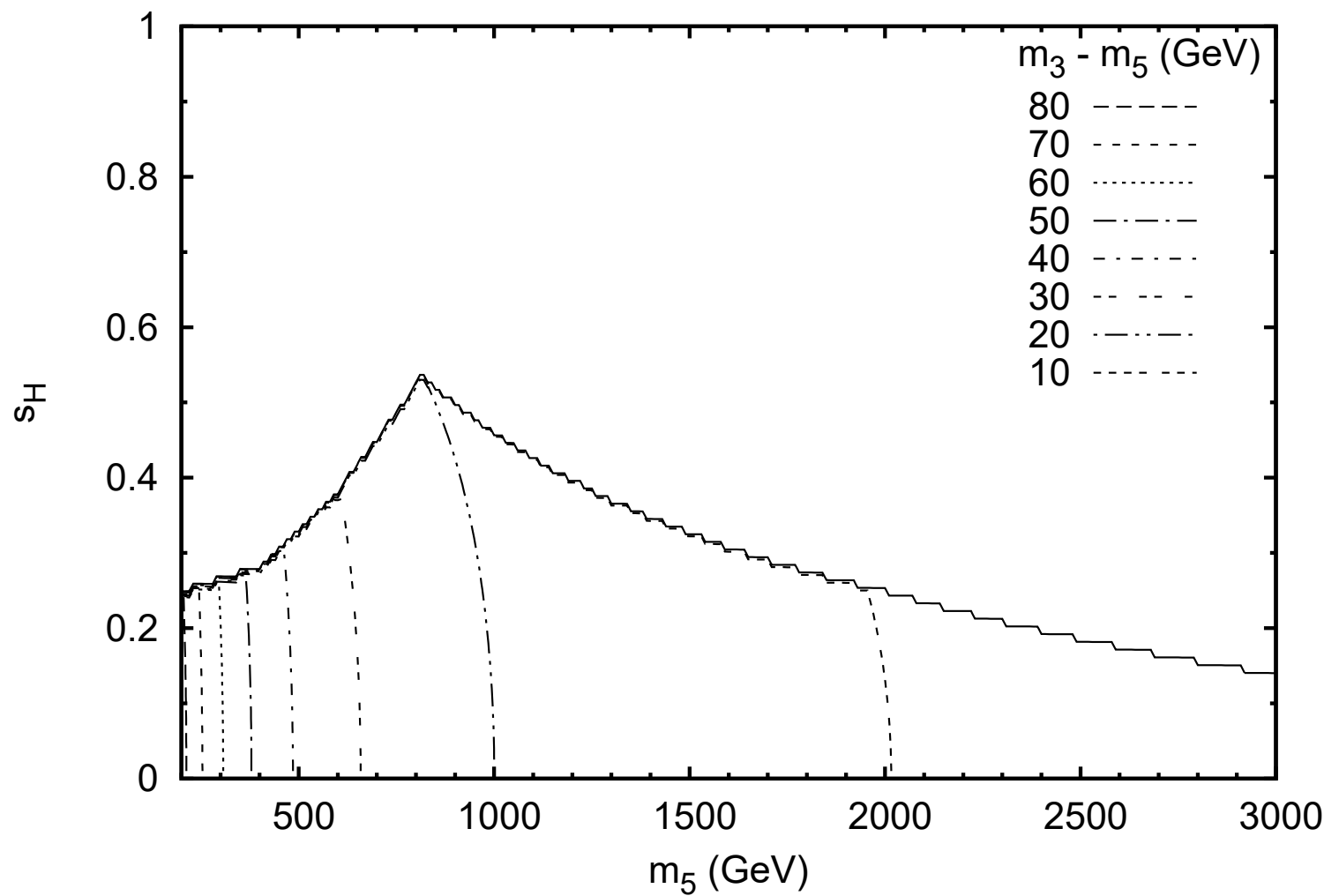

Figure 6: Contours of $m_{3}-m_{5}$ in the H5plane benchmark. The value of $m_{3}-m_{5}$ ranges from $6.7 \mathrm{GeV}$ to $84 \mathrm{GeV}$.

\section{$3.2 \quad H_{3}-H_{5}$ mass splitting}

In Fig. 6 we show the mass splitting $m_{3}-m_{5}$ in the H5plane benchmark. This splitting depends mainly on $m_{5}$, and varies from $84 \mathrm{GeV}$ at $m_{5}=200 \mathrm{GeV}$ to about $7 \mathrm{GeV}$ at $m_{5}=3000 \mathrm{GeV}$. In Fig. 7 we plot $m_{3}-m_{5}$ as a function of $m_{5}$ scanning over all the other free parameters in the H5plane benchmark (black points) and the full GM model (red points), where we have imposed the indirect constraints from $b \rightarrow s \gamma$ and the $S$ parameter [10] and direct constraints from the CMS search for $H_{5}^{ \pm \pm} \rightarrow W^{ \pm} W^{ \pm}$in vector boson fusion [11]. It is clear that the variation in the mass difference $m_{3}-m_{5}$ is much greater in the full model scan than it is in the H5plane benchmark. We can understand this as follows. 


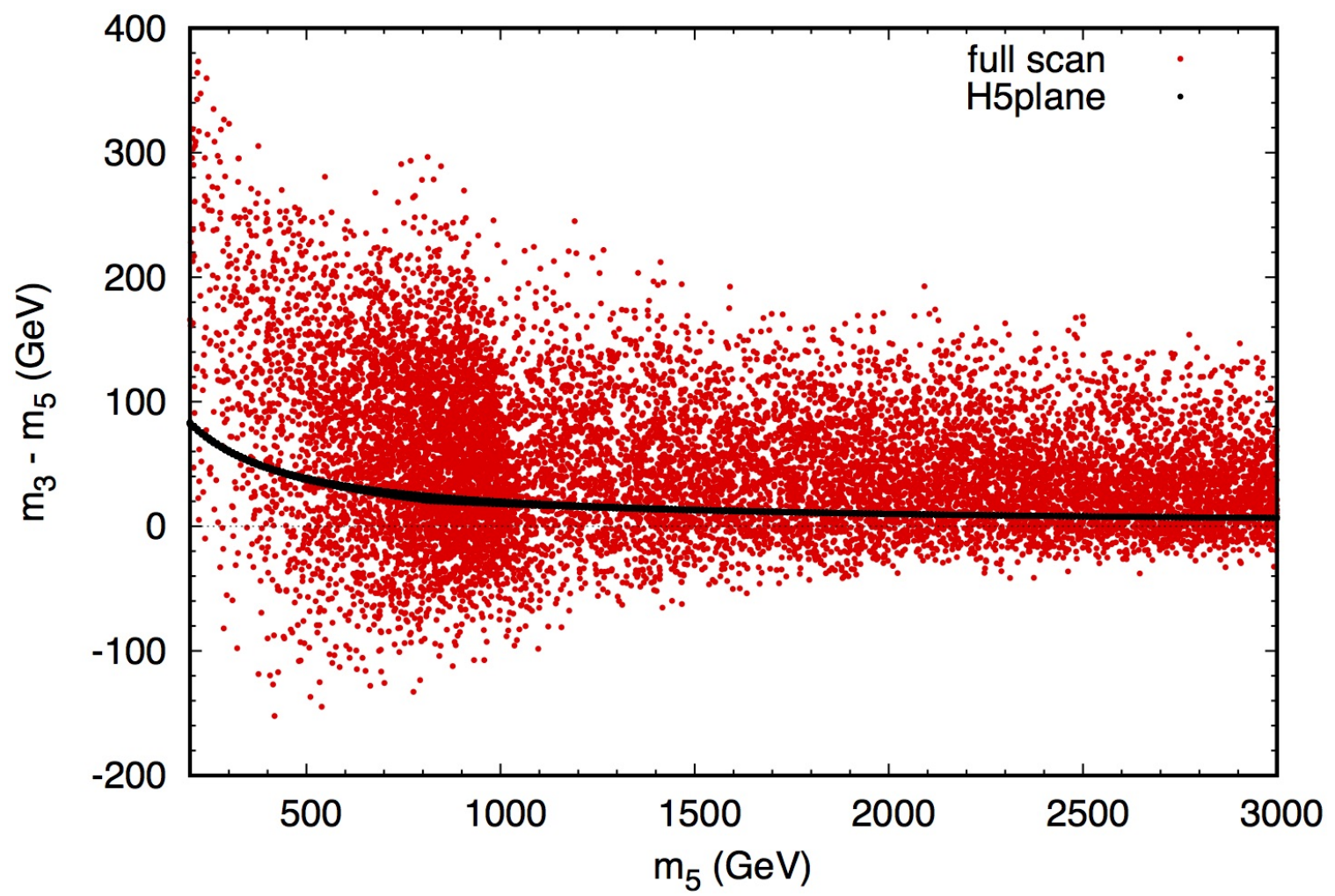

Figure 7: Mass difference $m_{3}-m_{5}$ as a function of $m_{5}$ in the H5plane benchmark (black points) and in a full scan of the GM model parameter space (red points). Indirect constraints from $b \rightarrow s \gamma$ and the $S$ parameter [10] and direct constraints from a CMS search for $H_{5}^{ \pm \pm} \rightarrow W^{ \pm} W^{ \pm}$in vector boson fusion [11] have been applied. 
The difference between $m_{3}^{2}$ and $m_{5}^{2}$ can be written in the full GM model as

$$
m_{3}^{2}-m_{5}^{2}=\left(M_{1}-6 M_{2}\right) \frac{s_{H} v}{\sqrt{2}}+\left[\lambda_{5}\left(\frac{1}{2} s_{H}^{2}-c_{H}^{2}\right)-\lambda_{3} s_{H}^{2}\right] v^{2} .
$$

In the H5plane benchmark, the parameter relations simplify this down to

$$
m_{3}^{2}-m_{5}^{2}=\left(m_{3}-m_{5}\right)\left(m_{3}+m_{5}\right)=\left(\frac{2}{3}-\frac{0.3 s_{H}^{2}}{c_{H}^{2}}\right) v^{2} .
$$

The variation of this expression with $s_{H}$ is fairly minimal: $m_{3}^{2}-m_{5}^{2}$ changes by less than $10 \%$ between $s_{H}=0$ and $s_{H}=0.4$. This leads to the very narrow range of $m_{3}-m_{5}$ covered by the H5plane benchmark scan (black points) in Fig. 7. Solving Eq. (72) for $m_{3}-m_{5}$, the dependence on $m_{5}$ is due only to a factor of $1 /\left(m_{3}+m_{5}\right) \simeq$ $1 /\left(2 m_{5}\right)$

In contrast, in the full GM model scan (red points in Fig. 7), $m_{3}-m_{5}$ varies by hundreds of $\mathrm{GeV}$. This is mostly due to the term proportional to $\left(M_{1}-6 M_{2}\right)$ in Eq. (71), which is zero in the H5plane benchmark due to the choice $M_{2}=M_{1} / 6$, and the term $-\lambda_{5} c_{H}^{2} v^{2}$, which is not suppressed at small $s_{H}$. In the full GM model, $\lambda_{5}$ can vary between $-8 \pi / 3$ and $+8 \pi / 3$ [6], while in the H5plane benchmark Eq. (67) reduces to

$$
\lambda_{5}=-\frac{2}{3 c_{H}^{2}}\left(1-0.1 s_{H}^{2}\right)
$$

so that the term $-\lambda_{5} c_{H}^{2} v^{2}$ varies from $2 v^{2} / 3$ by less than $2 \%$ for $s_{H}$ between zero and 0.4 in the H5plane benchmark. The preference for positive values of $m_{3}-m_{5}$ in the full GM model scan is due to the interplay of the theoretical constraints on the model parameters and is apparent already in Fig. 3 of Ref. [10]. Viable mass spectra in the full GM model, and their implications for cascade decays of the heavier Higgs bosons, have previously been studied in Ref. [17]. 


\subsection{Couplings and decays of $h$}

The tree-level couplings of the $125 \mathrm{GeV}$ Higgs boson $h$ in the GM model are given in terms of the underlying parameters by

$$
\kappa_{f}^{h}=\frac{c_{\alpha}}{c_{H}}, \quad \kappa_{V}^{h}=c_{\alpha} c_{H}-\sqrt{\frac{8}{3}} s_{\alpha} s_{H},
$$

where $\kappa$ is defined in the usual way as the ratio of the particle coupling in the GM model to the corresponding coupling of the SM Higgs boson [18].

We first illustrate the variation of the custodial-singlet scalar mixing angle $\sin \alpha$ over the H5plane benchmark in Fig. 8. $\sin \alpha$ varies between zero and -0.64 in the H5plane benchmark. It is strongly correlated with $s_{H}$, as shown in Fig. 9. This correlation also appears in a full scan of the GM model (red points in Fig. 9), but is stronger in the H5plane benchmark.

We plot $\kappa_{f}^{h}$ in the H5plane benchmark in Fig. 10, and we plot $\kappa_{V}^{h}$ in the H5plane benchmark in Fig. 11. These couplings remain reasonably close to their SM value of 1 everywhere in the benchmark plane. The coupling of $h$ to fermions $\kappa_{f}^{h}$ varies between 0.902 and 1.014, reaching its smallest values when $s_{H}$ is large, and the coupling of $h$ to vector bosons $\kappa_{V}^{h}$ varies between 1 and 1.21, reaching its largest values when $s_{H}$ is large.

The coupling of $h$ to photon pairs is affected by the modifications of these treelevel couplings, as well as by contributions from loop diagrams involving $H_{3}^{ \pm}, H_{5}^{ \pm}$, and $H_{5}^{ \pm \pm}$. Defining $\kappa_{\gamma}^{h}$ in the usual way as $[18]^{5}$

$$
\kappa_{\gamma}^{h}=\sqrt{\frac{\Gamma(h \rightarrow \gamma \gamma)}{\Gamma\left(h_{\mathrm{SM}} \rightarrow \gamma \gamma\right)}},
$$

we plot this coupling in the H5plane benchmark in Fig. 12. The coupling of $h$ to

\footnotetext{
${ }^{5}$ In GMCALC 1.2.1 the computation of the fermion loop contribution to Higgs decays to two photons includes only the top quark loop.
} 


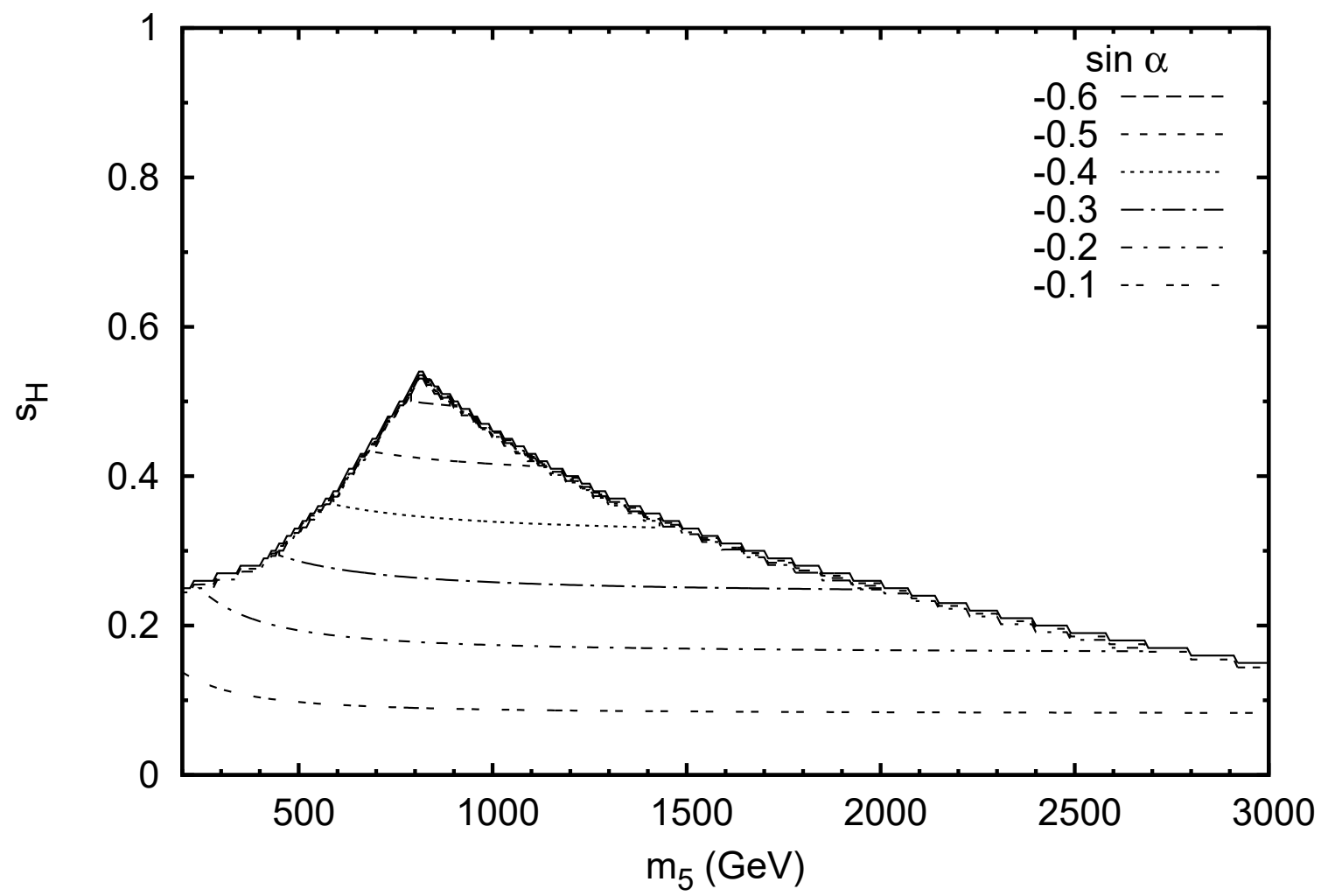

Figure 8: Contours of $\sin \alpha$ in the H5plane benchmark. The value of $\sin \alpha$ varies between -0.64 and 0 . 


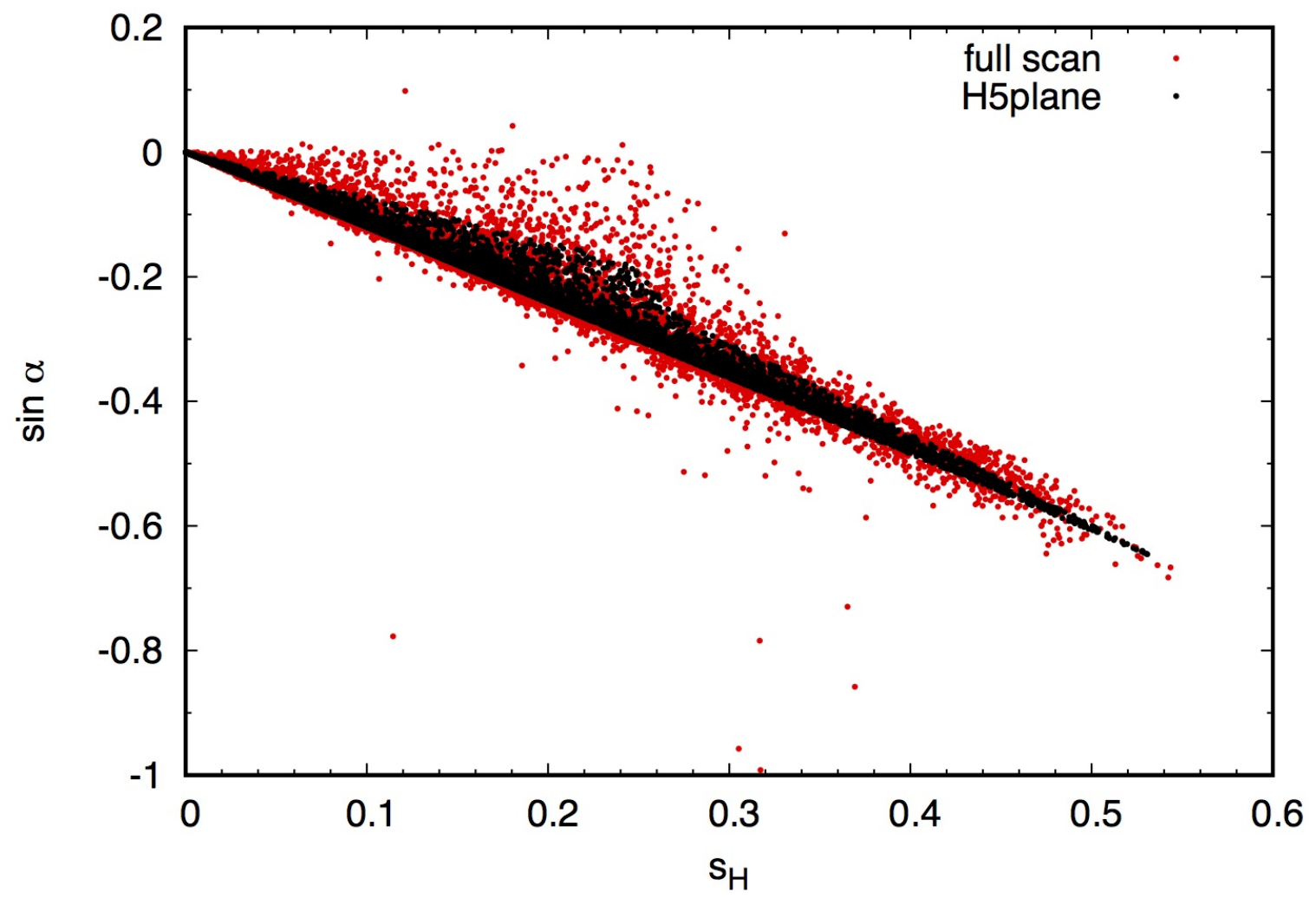

Figure 9: Correlation between $\sin \alpha$ and $s_{H}$ in the H5plane benchmark (black points) and in a general GM model scan with $m_{5} \geq 200 \mathrm{GeV}$ (red points). Indirect constraints from $b \rightarrow s \gamma$ and the $S$ parameter [10] and direct constraints from a CMS search for $H_{5}^{ \pm \pm} \rightarrow W^{ \pm} W^{ \pm}$in vector boson fusion [11] have been applied. 


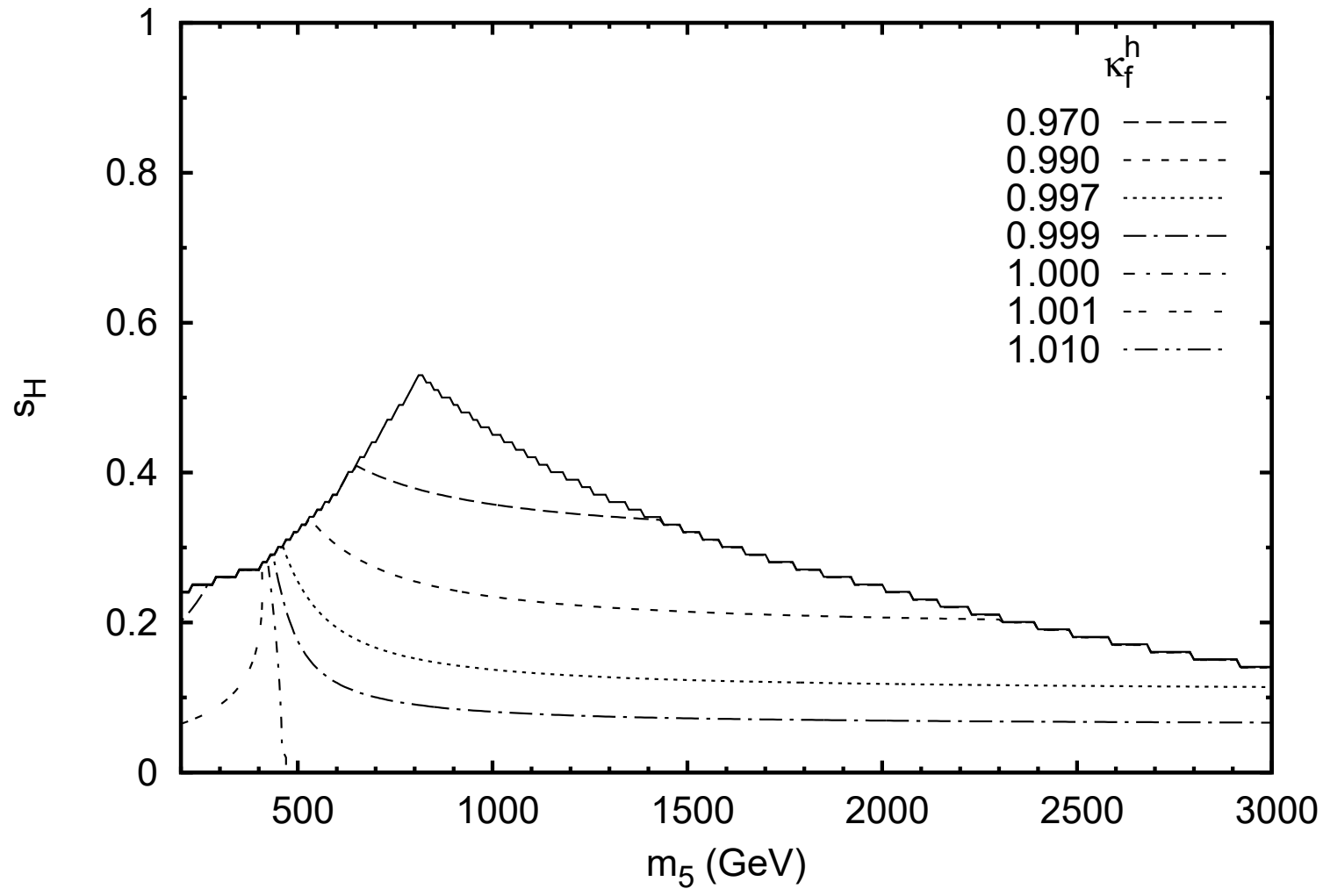

Figure 10: Contours of $\kappa_{f}^{h}$ in the H5plane benchmark. The value of $\kappa_{f}^{h}$ ranges from 0.902 to 1.014 . 


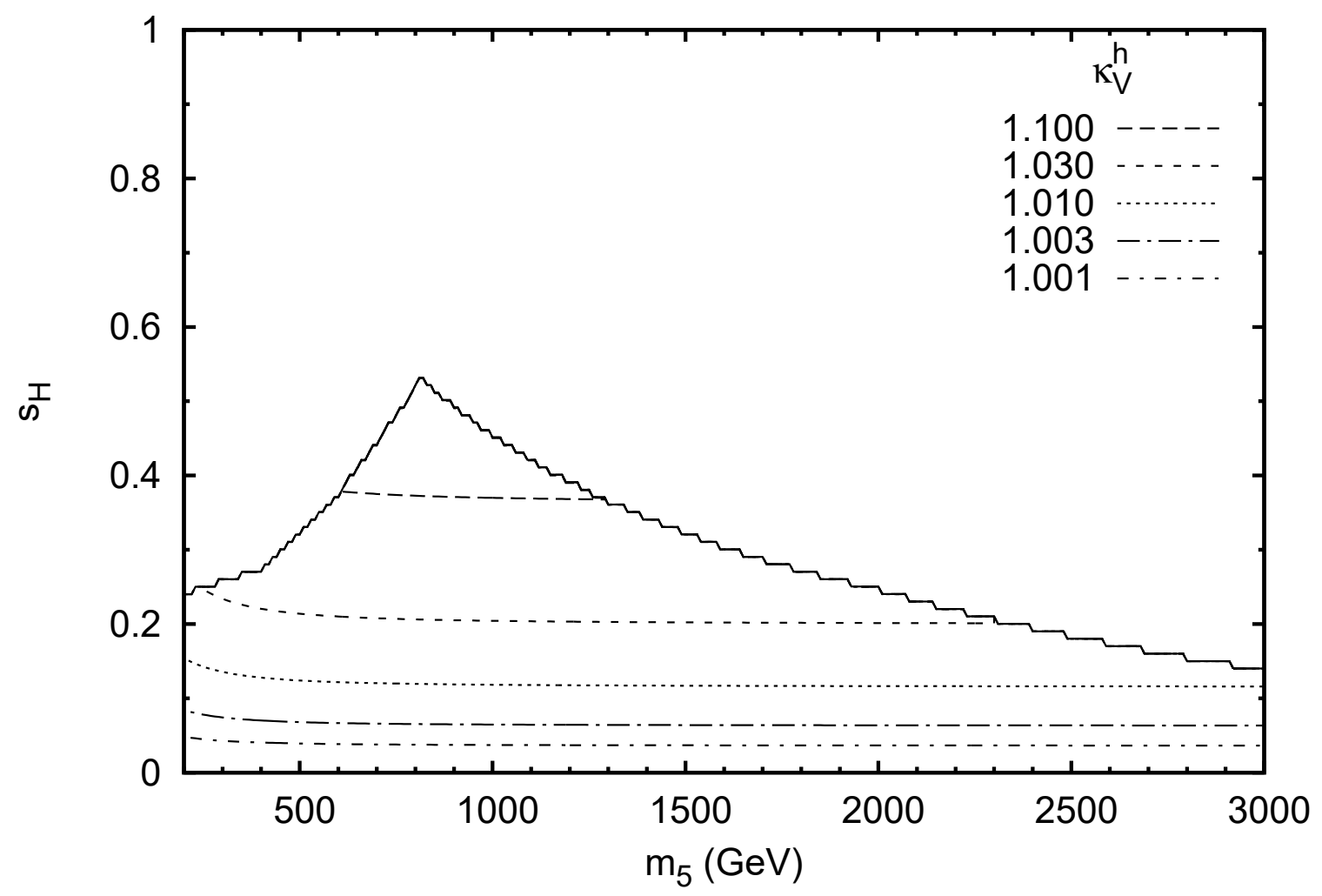

Figure 11: Contours of $\kappa_{V}^{h}$ in the H5plane benchmark. The value of $\kappa_{V}^{h}$ ranges from 1.00 to 1.21 . 


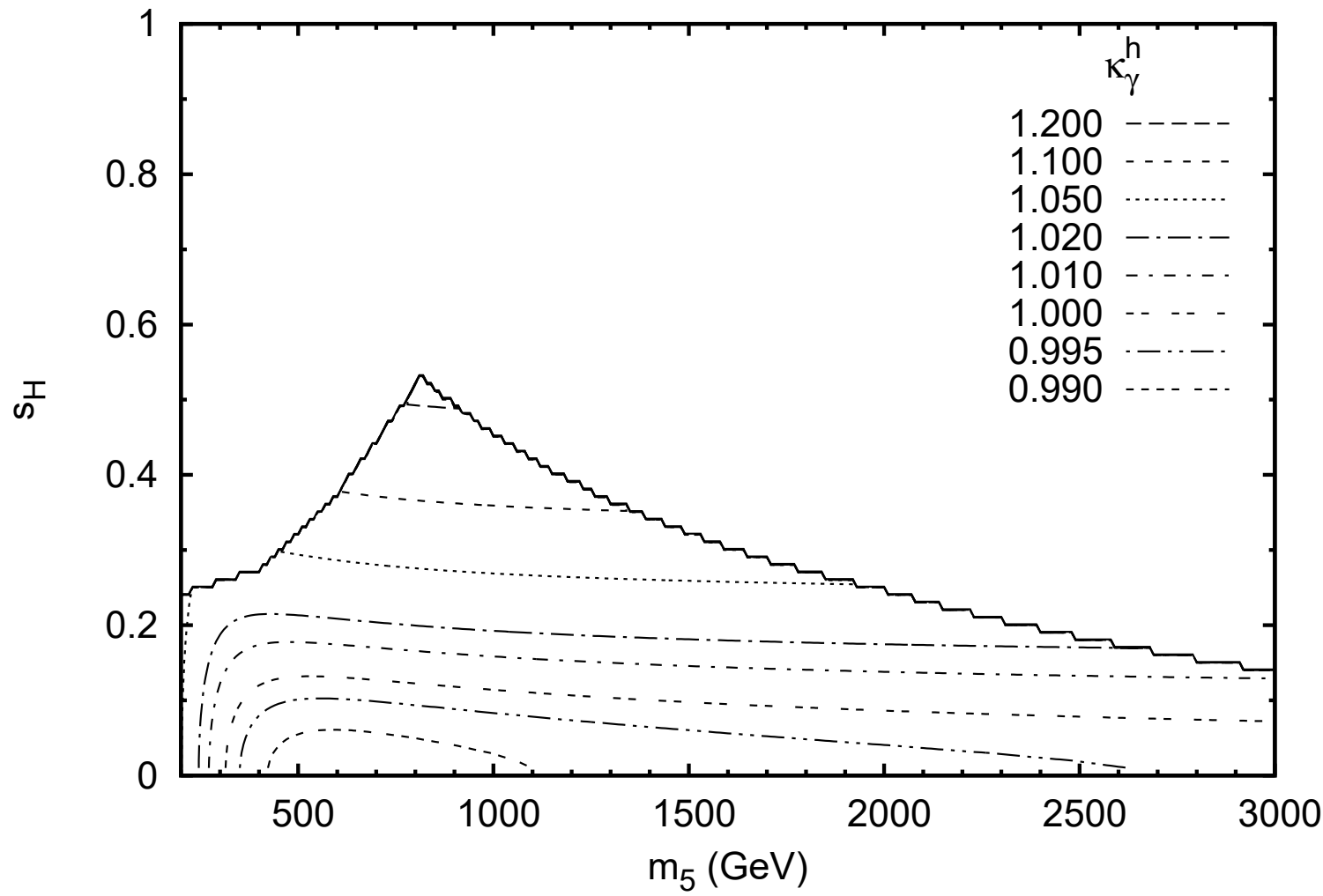

Figure 12: Contours of $\kappa_{\gamma}^{h}$ in the H5plane benchmark. The value of $\kappa_{\gamma}^{h}$ ranges from 0.987 to 1.24 .

photons $\kappa_{\gamma}^{h}$ varies between 0.99 and 1.24, reaching its largest values when $s_{H}$ is large. To isolate the effect of the loop diagrams involving $H_{3}^{ \pm}, H_{5}^{ \pm}$, and $H_{5}^{ \pm \pm}$, in Fig. 13 we plot $\Delta \kappa_{\gamma}^{h}$, which is defined as the contribution to $\kappa_{\gamma}^{h}$ made by the scalar loops, i.e.,

$$
\Delta \kappa_{\gamma}^{h}=\kappa_{\gamma}^{h}(\text { full })-\kappa_{\gamma}^{h}(t \text { and } W \text { loops only }) .
$$

$\Delta \kappa_{\gamma}^{h}$ varies between \pm 0.05 in the H5plane benchmark. It is positive only for $m_{5}$ below $300 \mathrm{GeV}$, where it contributes to a slight enhancement of $\kappa_{\gamma}^{h}$ to values up to 1.05 . It reaches its most negative value at large $s_{H}$, where it limits the enhancement of $\kappa_{\gamma}^{h}$ through destructive interference with the dominant $W$ loop contribution.

We also examine the total width of $h$ in the H5plane benchmark. We define the 


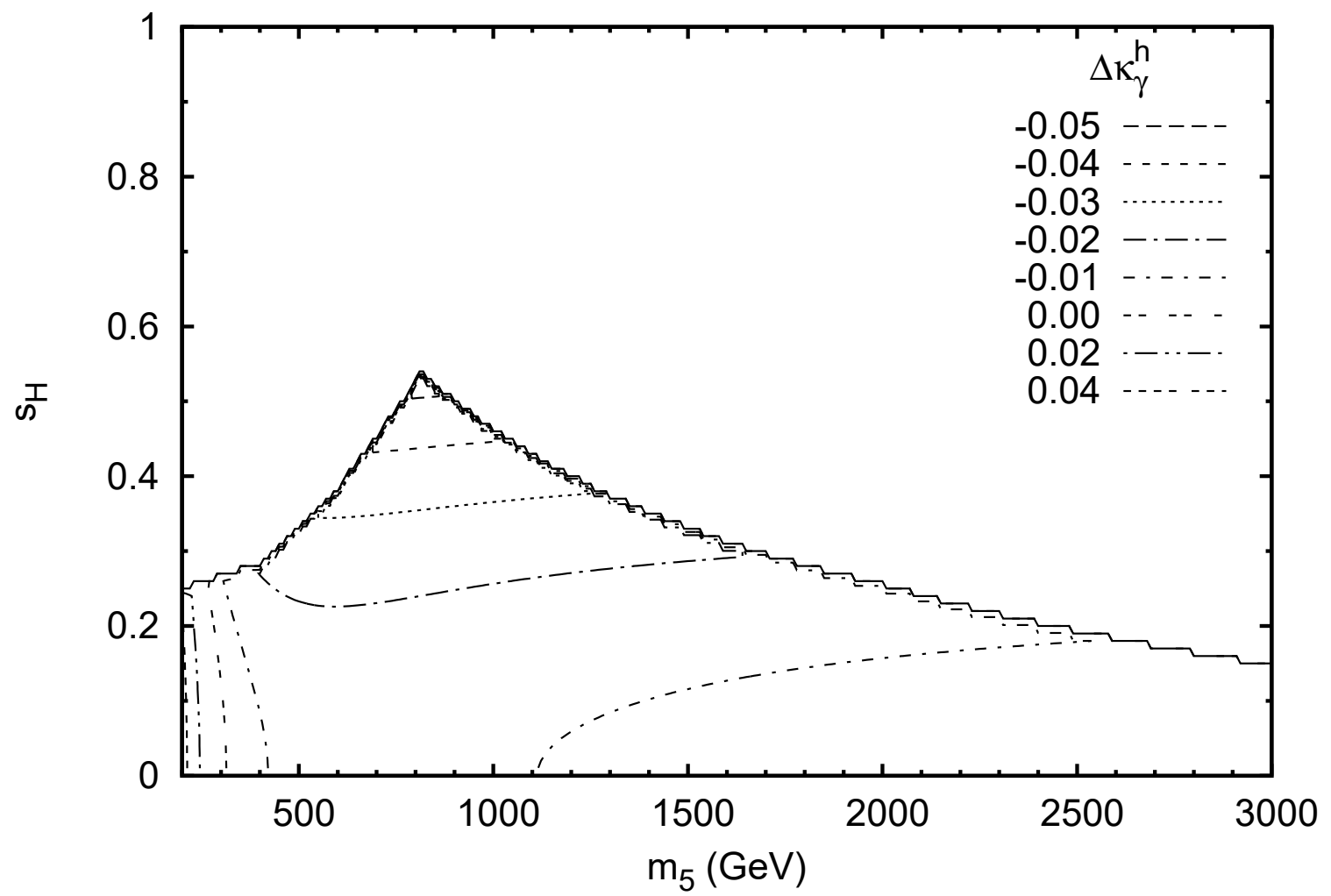

Figure 13: Contours of $\Delta \kappa_{\gamma}^{h}$ in the H5plane benchmark. The value of $\Delta \kappa_{\gamma}^{h}$ ranges from -0.054 to 0.052 . 


\begin{tabular}{cc}
\hline \hline branching ratio & value \\
\hline$B_{h \rightarrow b \bar{b}}^{S M}$ & $5.809 \times 10^{-1}$ \\
$B_{h \rightarrow \tau^{+} \tau^{-}}^{S M}$ & $6.256 \times 10^{-2}$ \\
$B_{h \rightarrow c \bar{c}}^{S M}$ & $2.884 \times 10^{-2}$ \\
$B_{h \rightarrow g g}^{S M}$ & $8.180 \times 10^{-2}$ \\
$B_{h \rightarrow W}^{S M}$ & $2.152 \times 10^{-1}$ \\
$B_{h \rightarrow Z Z}^{S M} W^{-}$ & $2.641 \times 10^{-2}$ \\
$B_{h \rightarrow \gamma \gamma}^{S M}$ & $2.270 \times 10^{-3}$ \\
$B_{h \rightarrow \gamma Z}^{S M}$ & $1.541 \times 10^{-3}$ \\
\hline \hline
\end{tabular}

Table 4: Branching ratios of the SM Higgs boson with mass $125.09 \mathrm{GeV}$, from Ref. [9], used in the calculation of $\kappa_{h}$.

scaling factor $\kappa_{h}$ as [18]

$$
\kappa_{h}=\sqrt{\frac{\Gamma_{\mathrm{tot}}(h)}{\Gamma_{\mathrm{tot}}\left(h_{\mathrm{SM}}\right)}},
$$

and calculate it using the formula

$$
\begin{gathered}
\kappa_{h}^{2}=\frac{1}{B_{t o t}^{S M}}\left[\left(\kappa_{f}^{h}\right)^{2}\left(B_{h \rightarrow b \bar{b}}^{S M}+B_{h \rightarrow \tau^{+} \tau^{-}}^{S M}+B_{h \rightarrow c \bar{c}}^{S M}+B_{h \rightarrow g g}^{S M}\right)\right. \\
\left.+\left(\kappa_{V}^{h}\right)^{2}\left(B_{h \rightarrow W^{+} W^{-}}^{S M}+B_{h \rightarrow Z Z}^{S M}\right)+\left(\kappa_{\gamma}^{h}\right)^{2} B_{h \rightarrow \gamma \gamma}^{S M}+\left(\kappa_{Z \gamma}^{h}\right)^{2} B_{h \rightarrow \gamma Z}^{S M}\right], \\
B_{t o t}^{S M}=B_{h \rightarrow b \bar{b}}^{S M}+B_{h \rightarrow \tau^{+} \tau^{-}}^{S M}+B_{h \rightarrow c \bar{c}}^{S M}+B_{h \rightarrow g g}^{S M} \\
+B_{h \rightarrow W^{+} W^{-}}^{S M}+B_{h \rightarrow Z Z}^{S M}+B_{h \rightarrow \gamma \gamma}^{S M}+B_{h \rightarrow \gamma Z}^{S M} .
\end{gathered}
$$

The values for the SM Higgs branching ratios $B_{h \rightarrow X}^{S M}$ were taken from Tables 174-178 of Ref. [9] for a SM Higgs mass of $125.09 \mathrm{GeV}$ and are reproduced in Table 4. We use this more precise value of the SM Higgs boson mass in this calculation because the LHC Higgs coupling measurements in Ref. [19], to which we will compare the GM model predictions below, have been extracted for this mass value.

We plot $\kappa_{h}$ in the H5plane benchmark in Fig. 14. $\kappa_{h}$ remains very close to one over the entire benchmark, varying between 0.985 and 1.017, which is surprising considering that the tree-level couplings of $h$ to vector bosons are modified by as much as $21 \%$ and those of $h$ to fermions by as much as $10 \%$ compared to the SM Higgs couplings. The very SM-like values of the $h$ total width are due to an accidental 


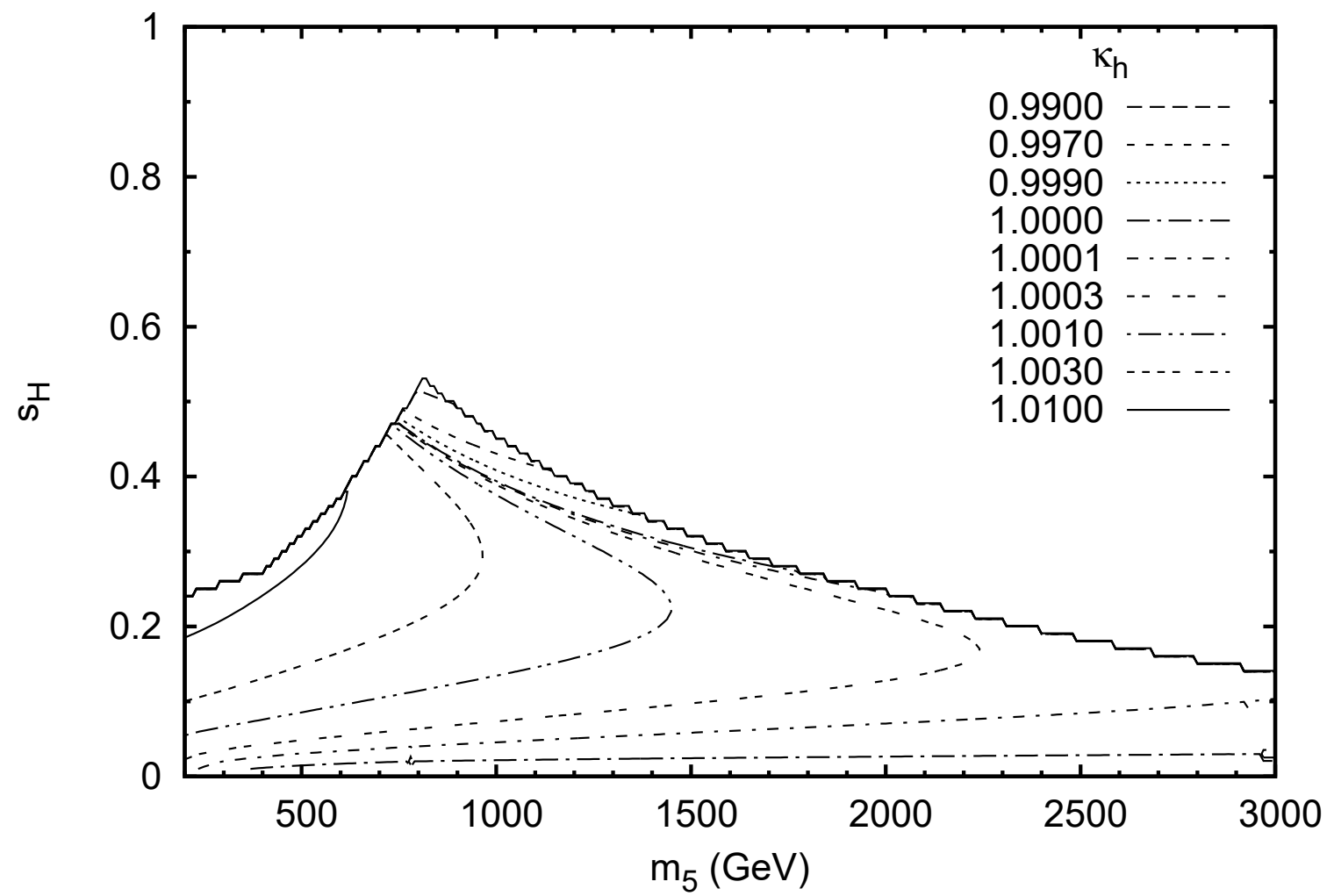

Figure 14: Contours of $\kappa_{h}$ in the H5plane benchmark. The value of $\kappa_{h}$ ranges from 0.985 to 1.017 .

cancellation between an enhancement of the $h$ partial width to vector bosons and a suppression of its partial width to fermions. This cancellation also occurs, though less severely, in a full scan of the GM model, as shown by the red points in Fig. 15. $\kappa_{h}$ is slightly greater than one in most of the H5plane benchmark, falling below one in a small sliver at high $s_{H}$ and $m_{5}$ between 700 and $1800 \mathrm{GeV}$, and in a thin band for $s_{H}<0.04$.

In order to evaluate the consistency of the H5plane benchmark with LHC measurements of the couplings of the $125 \mathrm{GeV}$ Higgs boson, we compute a $\chi^{2}$ using the combined ATLAS and CMS Higgs production and decay measurements in Ref. [19] from data collected at LHC centre-of-mass energies of 7 and $8 \mathrm{TeV}$. We use the observables and the corresponding correlation matrix $\rho$ summarized in Table 9 and Fig. 28, 


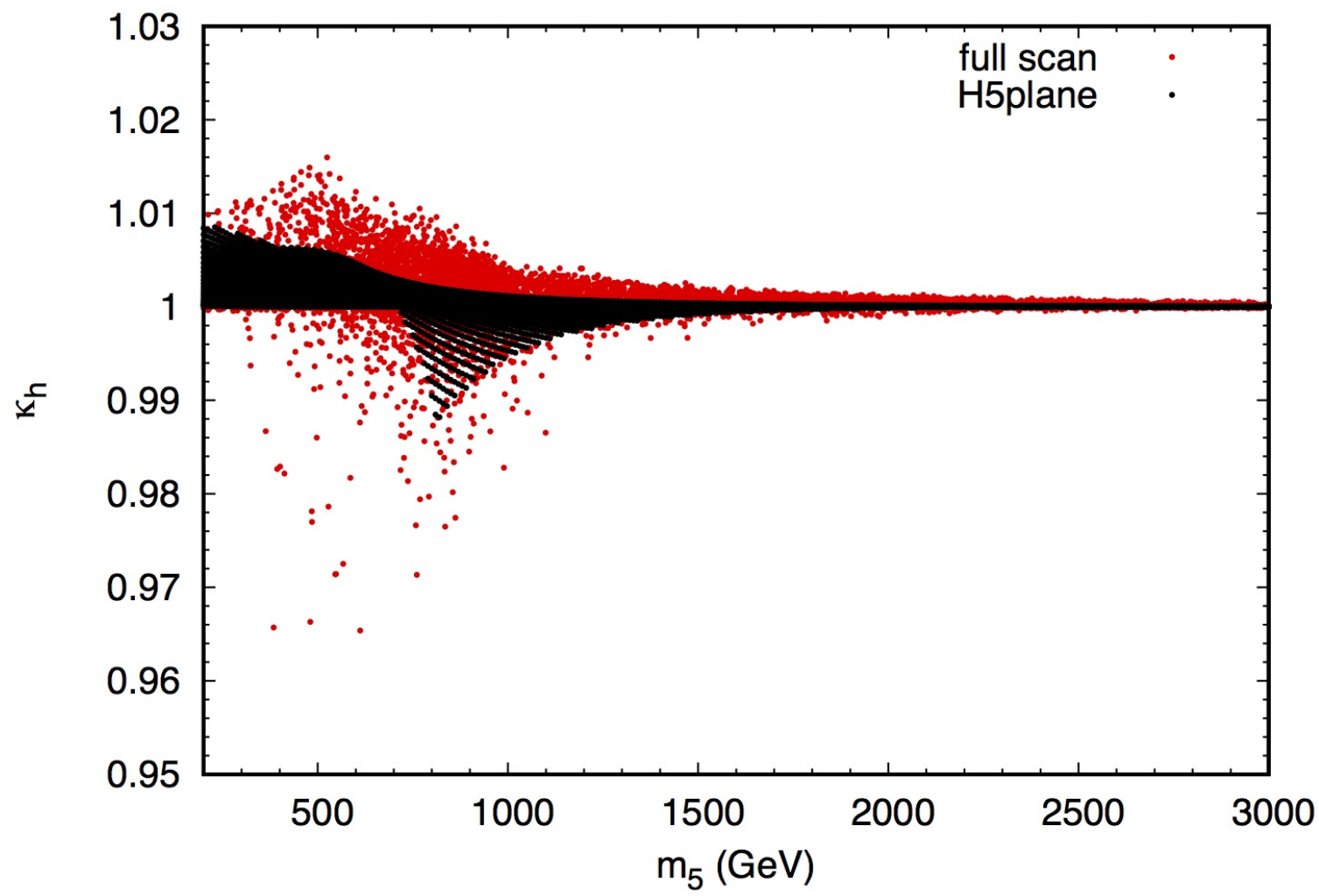

Figure 15: $\kappa_{h}$ as a function of $m_{5}$ in the H5plane benchmark (black points) and in a full scan of the GM model parameter space (red points). Indirect constraints from $b \rightarrow s \gamma$ and the $S$ parameter [10] and direct constraints from a CMS search for $H_{5}^{ \pm \pm} \rightarrow W^{ \pm} W^{ \pm}$in vector boson fusion [11] have been applied. 
respectively, of Ref. [19]. The $\chi^{2}$ is defined according to

$$
\chi^{2}=(\vec{x}-\vec{\mu})^{T} V^{-1}(\vec{x}-\vec{\mu}), \quad V_{i j}=\rho_{i j} \sigma_{i} \sigma_{j},
$$

where $\vec{x}$ is the vector of observed values, $\vec{\mu}$ is the vector of theoretical values at a particular point in the H5plane benchmark, and $\vec{\sigma}$ is the vector of the combined theoretical and experimental uncertainties. Where the experimental uncertainties in Table 9 of Ref. [19] are asymmetric, we symmetrize them by averaging the upper and lower uncertainty. We then combine the (symmetrized) experimental uncertainties with the theoretical uncertainties quoted in Table 9 of Ref. [19] by adding them in quadrature. ${ }^{6}$ The results are shown in Fig. 16. The $\chi^{2}$ in the H5plane benchmark of the GM model ranges from a maximum of 29.9 for $s_{H}$ near zero to a minimum of 16.2 for $s_{H}$ around 0.5 and $m_{5}$ around $800-1000 \mathrm{GeV}$. For comparison, the $\chi^{2}$ for the SM Higgs, computed in the same way, is 29.4. The lower $\chi^{2}$ values in the GM model reflect a pull in the data towards slightly lower $\kappa_{f}^{h}$ and higher $\kappa_{V}^{h}$ values. In particular, we observe that the entire H5plane benchmark is currently consistent with LHC Higgs coupling data.

However, examination of Fig. 8 of Ref. [19] shows that variation in the measurement of $B^{b b} / B^{Z Z}$ is lopsided and fat-tailed, contributing to the high values of $\chi^{2}$ with both the Standard Model and the H5plane benchmark. To compensate for this, we take some visual measurements from Fig. 8 of Ref. [19] and take a quadratic fit to the relationship between standard deviations and distance on the upper experimental error in $B^{b b} / B^{Z Z}$. This tells us that the deviation of the Standard Model from the observed value is roughly 2.57 standard deviations; if the variation in $B^{b b} / B^{Z Z}$ were Gaussian, this would correspond to a standard deviation of 6.73 . Thus, we will assume that the standard deviation for $B^{b b} / B^{Z Z}$ is in fact 6.73 , rather than the aver-

\footnotetext{
${ }^{6}$ The SM theoretical predictions and uncertainties were multiplied by appropriate kappa factors to get the GM model theoretical predictions and uncertainties.
} 


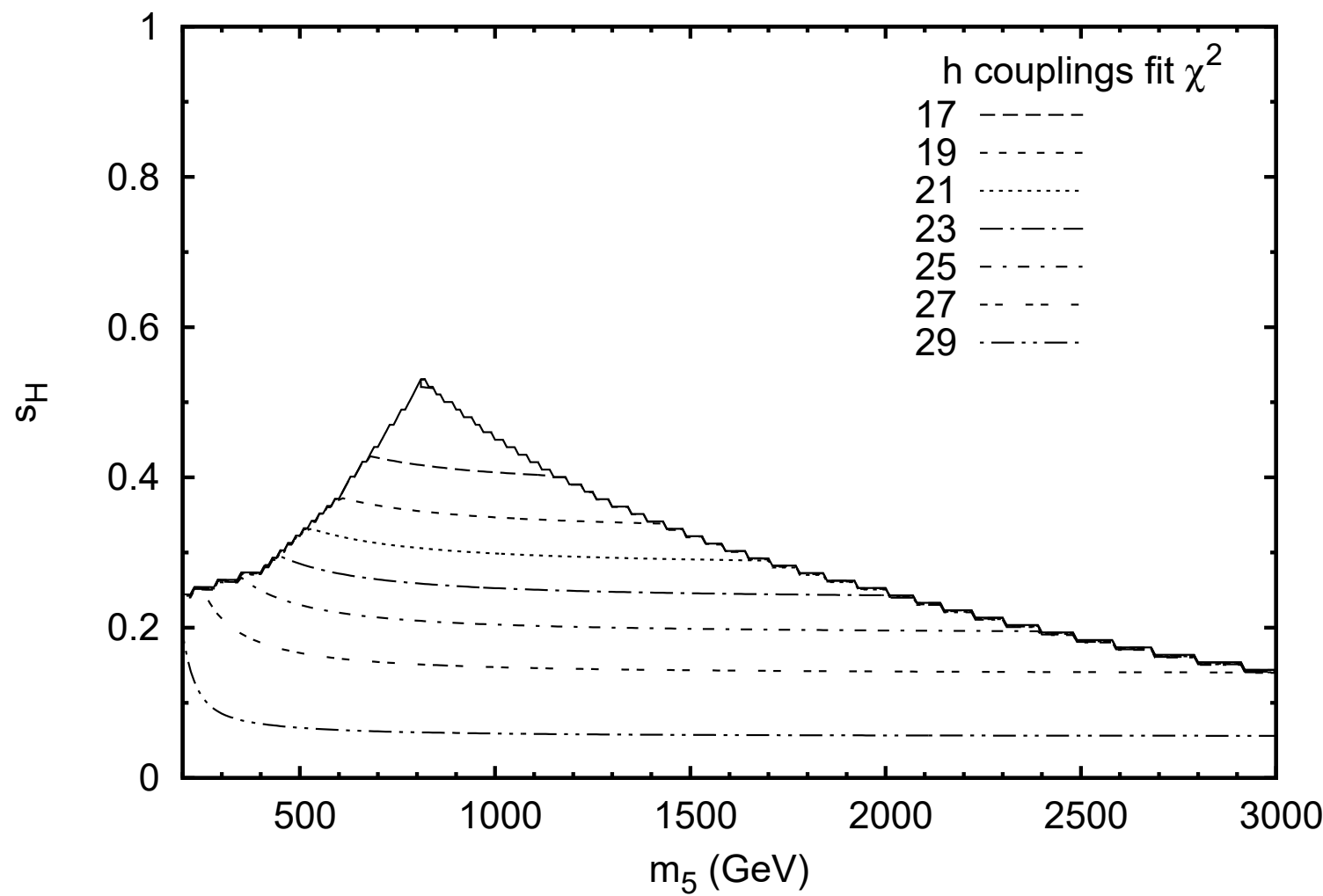

Figure 16: Contours of the $\chi^{2}$ value for a fit of the $h$ cross sections and branching ratios in the H5plane benchmark to LHC Higgs boson measurements from Ref. [19]. The $\chi^{2}$ ranges from 16.2 to 29.9. Compare the $\chi^{2}$ of 29.4 for the SM Higgs boson. 


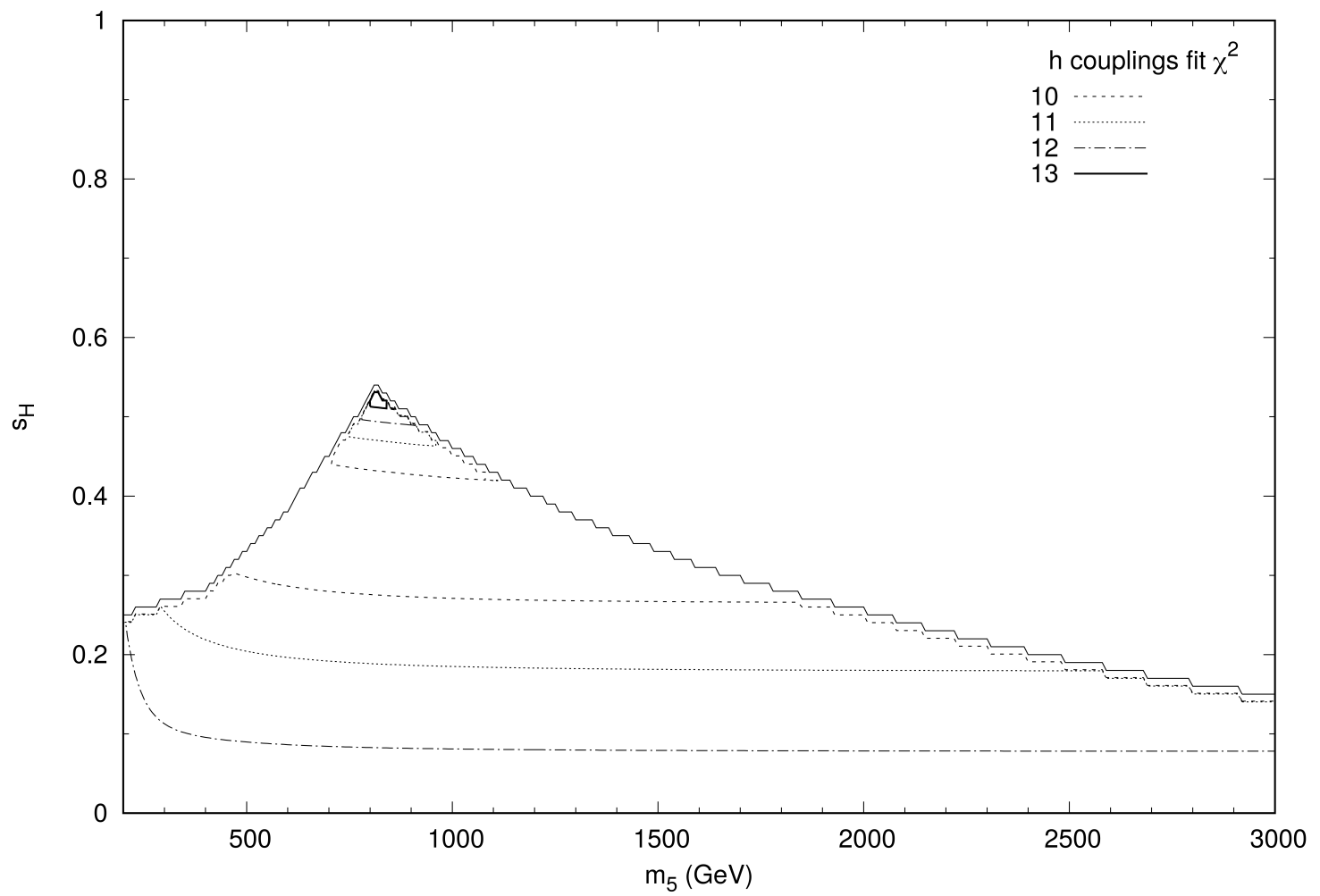

Figure 17: Contours of the $\chi^{2}$ value for a fit of the $h$ cross sections and branching ratios in the H5plane benchmark to LHC Higgs boson measurements from Ref. [19], with the standard deviation for $B^{b b} / B^{Z Z}$ set to 6.73 to account for a lopsided and fat-tailed distribution. The $\chi^{2}$ ranges from 9.44 to 14.29 . Compare the $\chi^{2}$ of 12.28 for the SM Higgs boson.

aged value of 3.5 that we had used previously. In this case, the SM value of $\chi^{2}$ drops to 12.3 , with a p-value of $19.8 \%$, which is much closer to what one would expect from a $\chi^{2}$ test of the standard model (according to the full likelihood analysis of Ref. [19], the compatability between the data and the SM predictions has a p-value of 16\%). Applying this test to the entire H5plane, we get $\chi^{2}$ values ranging from 9.44 to 14.3 , with p-values ranging from $39.8 \%$ to $11.2 \%$, as seen in Fig. 17.

\subsection{Couplings and decays of $H$}

We now examine the couplings and decays of the heavier custodial-singlet Higgs boson $H$. The tree-level couplings of $H$ in the GM model are given in terms of the underlying 
parameters by

$$
\kappa_{f}^{H}=\frac{s_{\alpha}}{c_{H}}, \quad \quad \kappa_{V}^{H}=s_{\alpha} c_{H}+\sqrt{\frac{8}{3}} c_{\alpha} s_{H},
$$

where the $\kappa$ factors are again defined as the ratio of the $H$ coupling in the GM model to the corresponding coupling of the SM Higgs boson, if the SM Higgs were as massive as the $H$. In Fig. 18 we plot $\kappa_{f}^{H}$ in the H5plane benchmark, and in Fig. 19 we plot $\kappa_{V}^{H}$ in the H5plane benchmark. These couplings are interesting mainly because they control the production of $H$ via gluon fusion ( $\mathrm{ggF}$, when two gluons collide, transform into virtual quarks, and become a Higgs) and vector boson fusion (VBF, where two fermions collide and exchange a virtual weak gauge boson, which emits a Higgs), respectively. The magnitude of the coupling of $H$ to fermions is largest at large $s_{H}$, reaching -0.76 times the corresponding SM Higgs coupling strength. The coupling of $H$ to vector boson pairs is largest at low $m_{5} \sim 200-300 \mathrm{GeV}$ and large $s_{H}$, reaching 0.22 times the corresponding SM Higgs coupling strength. Squaring these, the cross sections for $H$ production by gluon fusion and vector boson fusion reach at most 0.58 and 0.048 times the corresponding SM Higgs cross sections for a Higgs boson of the same mass as $H$, respectively. ${ }^{7}$

In Figs. 20, 21, 22, and 23 we plot the branching ratios of $H$ to $W^{+} W^{-}, Z Z$, $h h$, and $t \bar{t}$. These are the dominant decays of $H$ over the entire H5plane benchmark. The branching ratios of $H$ to $W^{+} W^{-}$and $Z Z$ dominate for $m_{5}$ below $600 \mathrm{GeV}$, with branching ratios above $40 \%$ and $20 \%$, respectively. These decays reach maximum branching ratios of $65 \%$ and $30 \%$, respectively, for low $m_{5} \sim 200-300 \mathrm{GeV}$. The branching ratio of $H$ to $W^{+} W^{-}(Z Z)$ remains above $20 \%(10 \%)$ over most of the benchmark plane, out to the highest $m_{5}$ values.

The branching ratio of $H$ to $h h$ dominates at high masses, reaching $50 \%$ for $m_{5} \sim$

\footnotetext{
${ }^{7}$ These SM cross sections can be found in Ref. [20]. For example, if we have $m_{H}=1000 \mathrm{GeV}$, then the SM cross section for VBF is $87.3 \mathrm{fb}$, and the SM cross section for ggF is $123 \mathrm{fb}$. If we choose $s_{H}=0.3$, then $m_{5}=976 \mathrm{GeV}, \kappa_{V}^{H}=0.125$, and $\kappa_{f}^{H}=-0.367$, in which case the cross sections for the $\mathrm{GM}$ scalar $H$ are $1.34 \mathrm{fb}$ for $\mathrm{VBF}$ and $16.6 \mathrm{fb}$ for $\mathrm{ggF}$.
} 


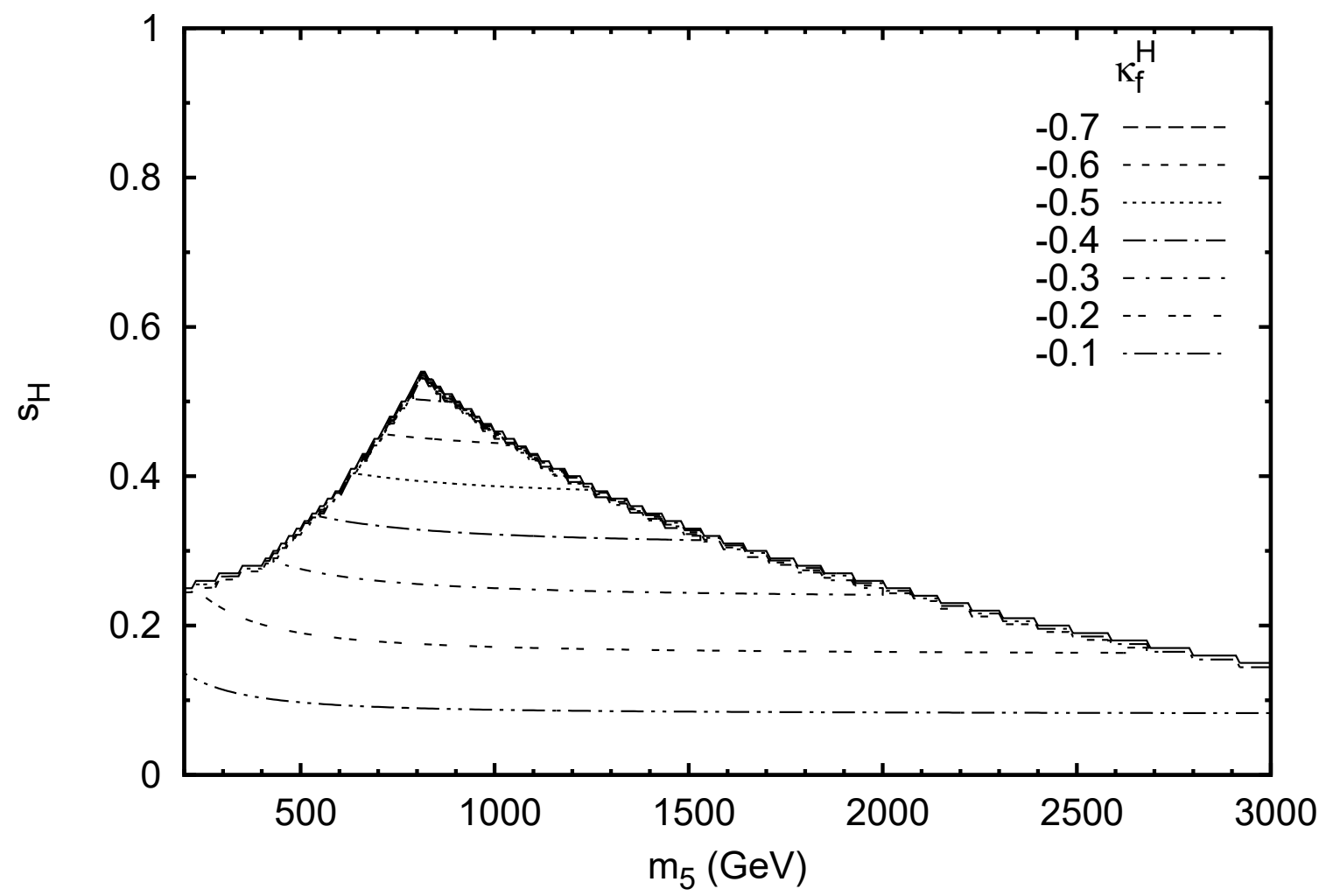

Figure 18: Contours of $\kappa_{f}^{H}$ in the H5plane benchmark. $\kappa_{f}^{H}$ ranges from zero to -0.76 . $1000 \mathrm{GeV}$ and a maximum of $71 \%$ for the highest $s_{H}$ values at large $m_{5}>1500 \mathrm{GeV}$. The branching ratio of $H$ to $t \bar{t}$ reaches a maximum of $37 \%$ for $m_{5} \sim 500-600 \mathrm{GeV}$ and high $s_{H}$, but falls below $10 \%$ for $m_{5} \gtrsim 1400 \mathrm{GeV}$. Note that, because $m_{H}>m_{5}$ in the H5plane benchmark, the kinematic threshold for $H \rightarrow t \bar{t}$ at $m_{H}=2 m_{t}$ occurs when $m_{5} \simeq 250 \mathrm{GeV}$.

\section{5 $\quad H-H_{5}$ mass splitting}

Decays of $H$ to $H_{5}^{ \pm} W^{\mp}$ and of $H$ to $H_{5}^{0} Z$ are forbidden by custodial symmetry. Therefore our interest in the mass splitting between $H$ and $H_{5}^{0}$ is due to the fact that both of these states can be produced in vector boson fusion with decays to $W^{+} W^{-}$ and $Z Z$, which opens the possibility of interference between their lineshapes if the resonances are close enough together. In Fig. 24 we show the mass splitting $m_{H}-m_{5}$ in the H5plane benchmark. The splitting varies from $120 \mathrm{GeV}$ at $m_{5}=200 \mathrm{GeV}$ to 


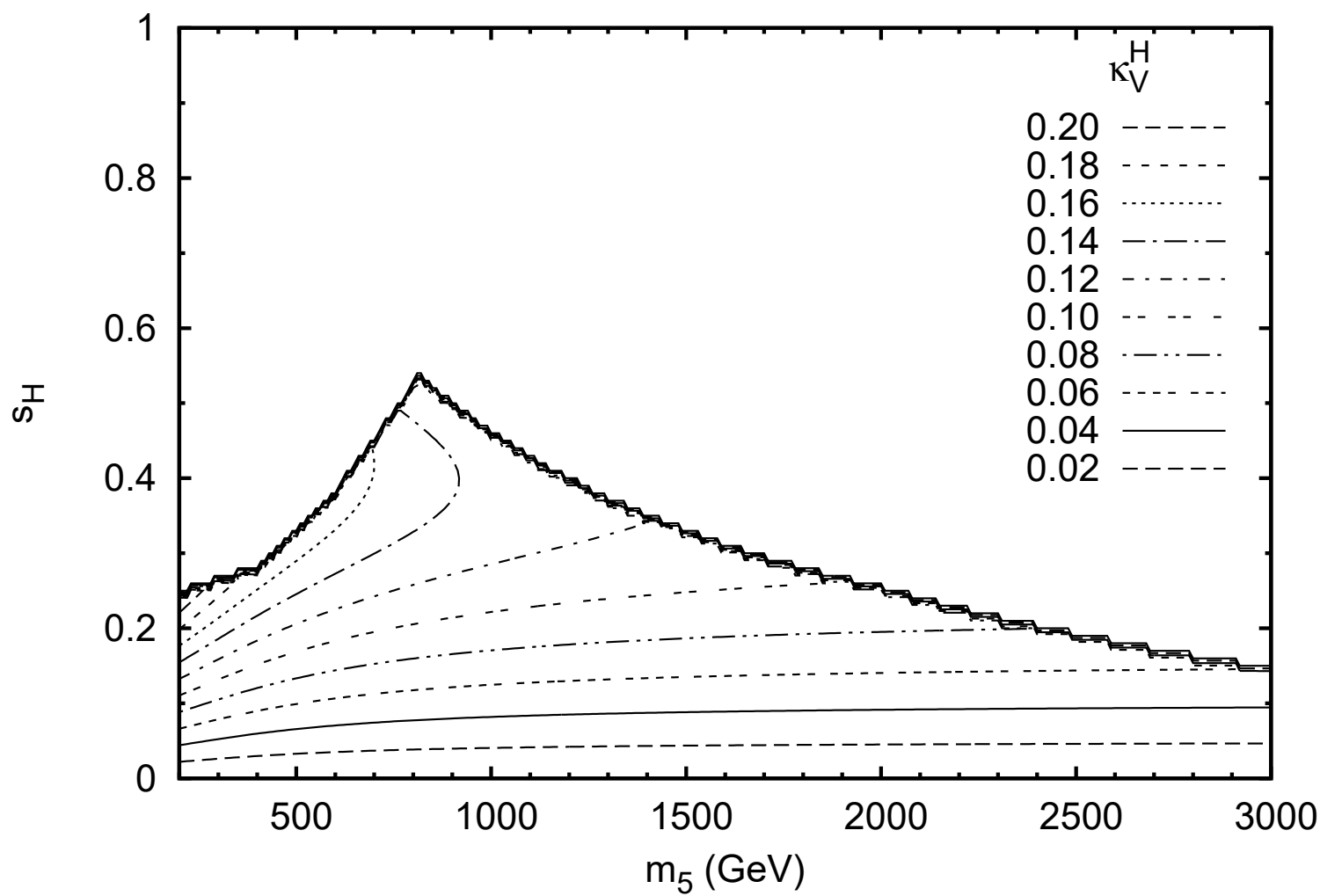

Figure 19: Contours of $\kappa_{V}^{H}$ in the H5plane benchmark. $\kappa_{V}^{H}$ ranges from zero to 0.22.

about $9 \mathrm{GeV}$ at $m_{5}=3000 \mathrm{GeV}$. In Fig. 25 we plot $m_{H}-m_{5}$ as a function of $m_{5}$ scanning over all the other free parameters in the H5plane benchmark (black points) and the full GM model (red points), where we have imposed the indirect constraints from $b \rightarrow s \gamma$ and the $S$ parameter [10] and direct constraints from the CMS search for $H_{5}^{ \pm \pm} \rightarrow W^{ \pm} W^{ \pm}$in vector boson fusion [11]. Similarly to the case of $m_{3}-m_{5}$, we see that the variation in the mass difference $m_{H}-m_{5}$ is much greater in the full model scan than it is in the H5plane benchmark.

To understand the experimental implications of this mass splitting, we compare it to the intrinsic widths ${ }^{8}$ of $H$ and $H_{5}^{0}$. In Figs. 26 and 27 we first plot the total width of $H$ (Fig. 26) and the ratio $\Gamma_{\text {tot }}(H) / \Gamma_{\text {tot }}\left(H_{5}^{0}\right)$ (Fig. 27) in the H5plane benchmark. The total widths of $H$ and $H_{5}^{0}$ are very similar for $m_{5} \gtrsim 500 \mathrm{GeV}$. For lower masses, the fact that $H$ is significantly heavier than $H_{5}^{0}$ allows its width to become more than

\footnotetext{
8 "Intrinsic width" is just the total width of the particle; it is expressed in GeV because the shorter a particle's lifetime, the more uncertainty there is in its mass when it appears.
} 


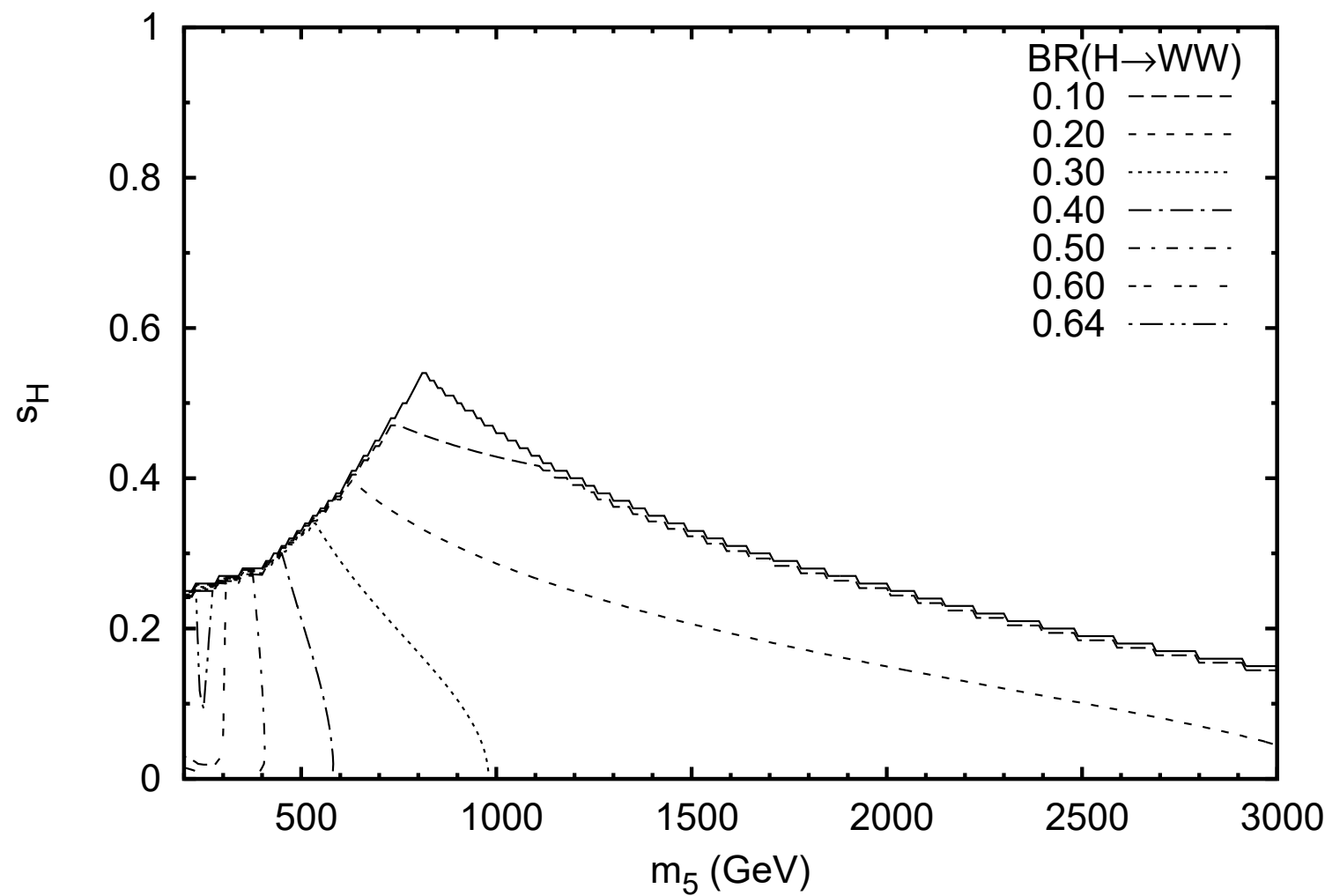

Figure 20: Contours of $\operatorname{BR}\left(H \rightarrow W^{+} W^{-}\right)$in the H5plane benchmark. $\operatorname{BR}(H \rightarrow$ $\left.W^{+} W^{-}\right)$ranges from 0.05 to 0.65 . 


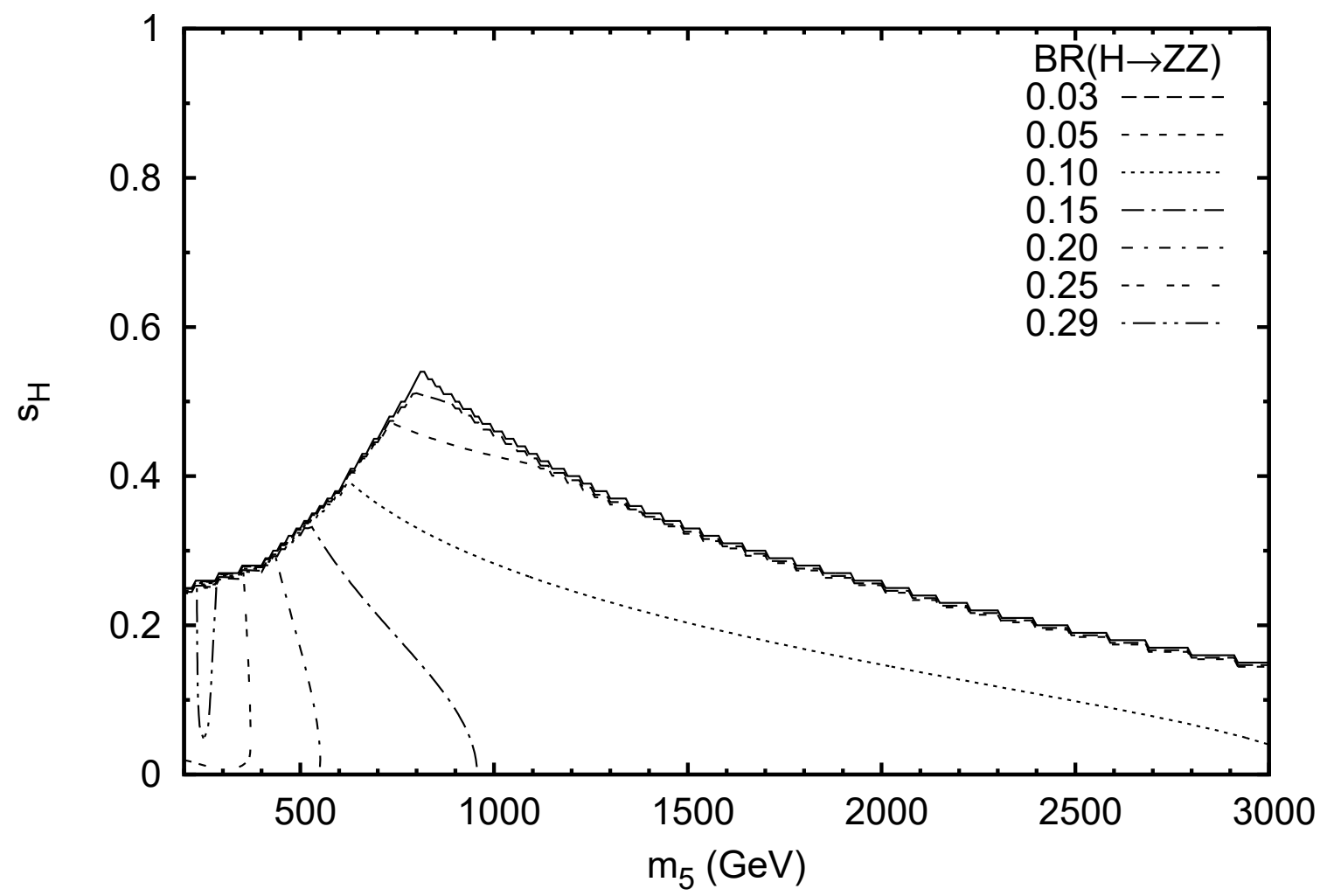

Figure 21: Contours of $\mathrm{BR}(H \rightarrow Z Z)$ in the H5plane benchmark. $\mathrm{BR}(H \rightarrow Z Z)$ ranges from 0.02 to 0.30 . 


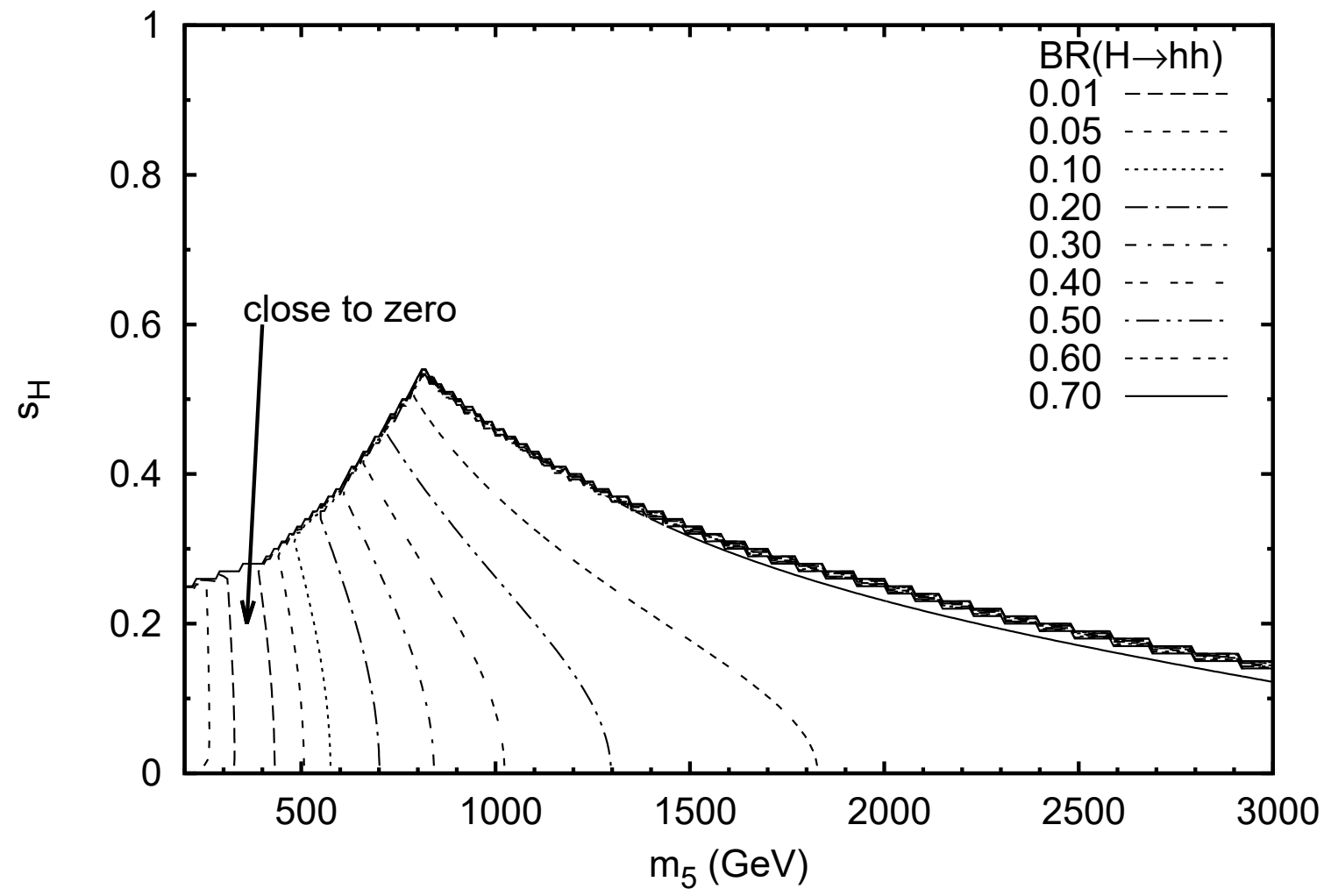

Figure 22: Contours of $\mathrm{BR}(H \rightarrow h h)$ in the H5plane benchmark. $\mathrm{BR}(H \rightarrow h h)$ ranges from zero to 0.71 . 


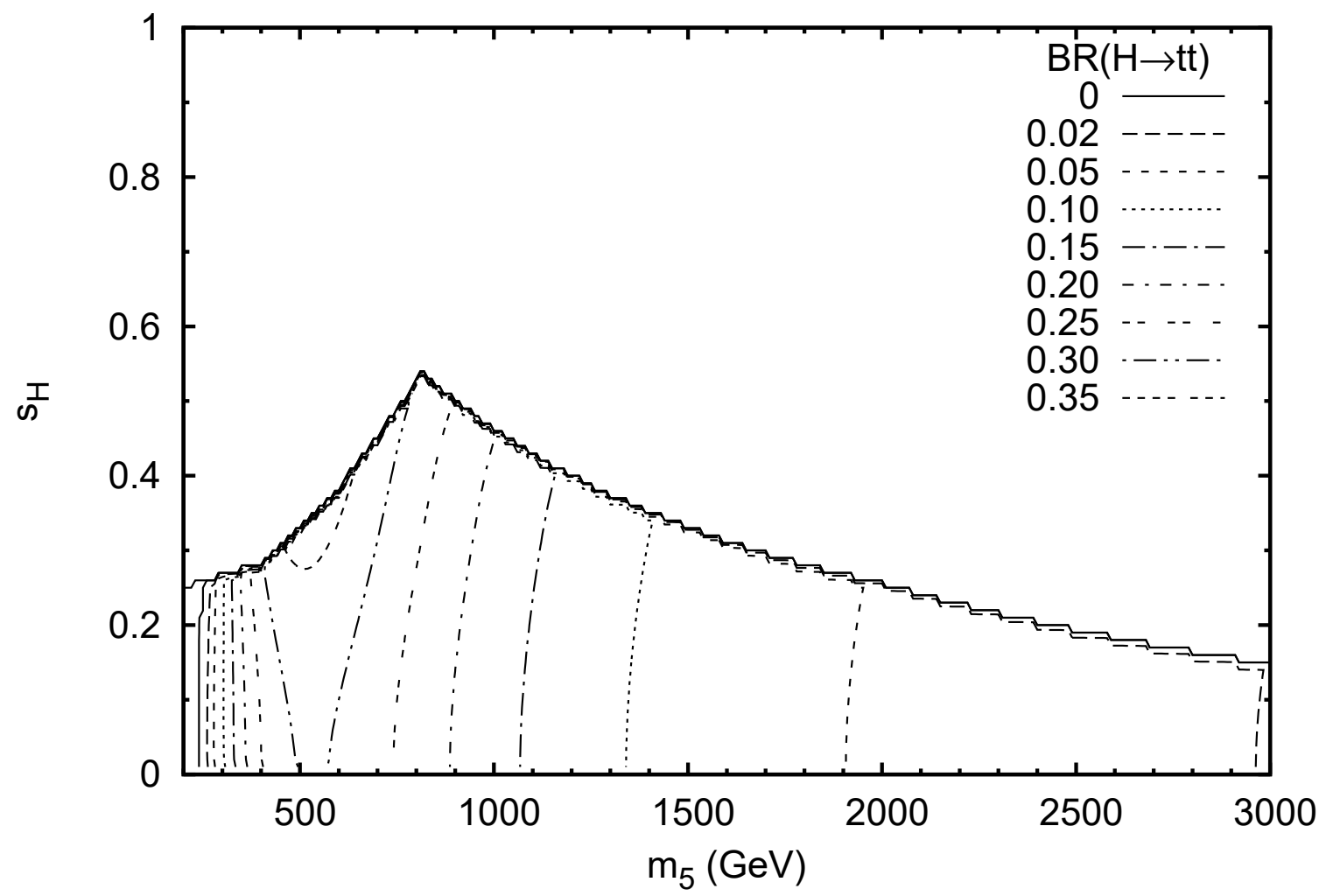

Figure 23: Contours of $\mathrm{BR}(H \rightarrow t \bar{t})$ in the $\mathrm{H} 5$ plane benchmark. $\mathrm{BR}(H \rightarrow t \bar{t})$ ranges from zero to 0.37 . $\mathrm{BR}(H \rightarrow t \bar{t})$ drops abruptly to zero when $m_{H}<2 m_{t}$ because off-shell decays to $t \bar{t}$ are not calculated in GMCALC 1.2.1. 


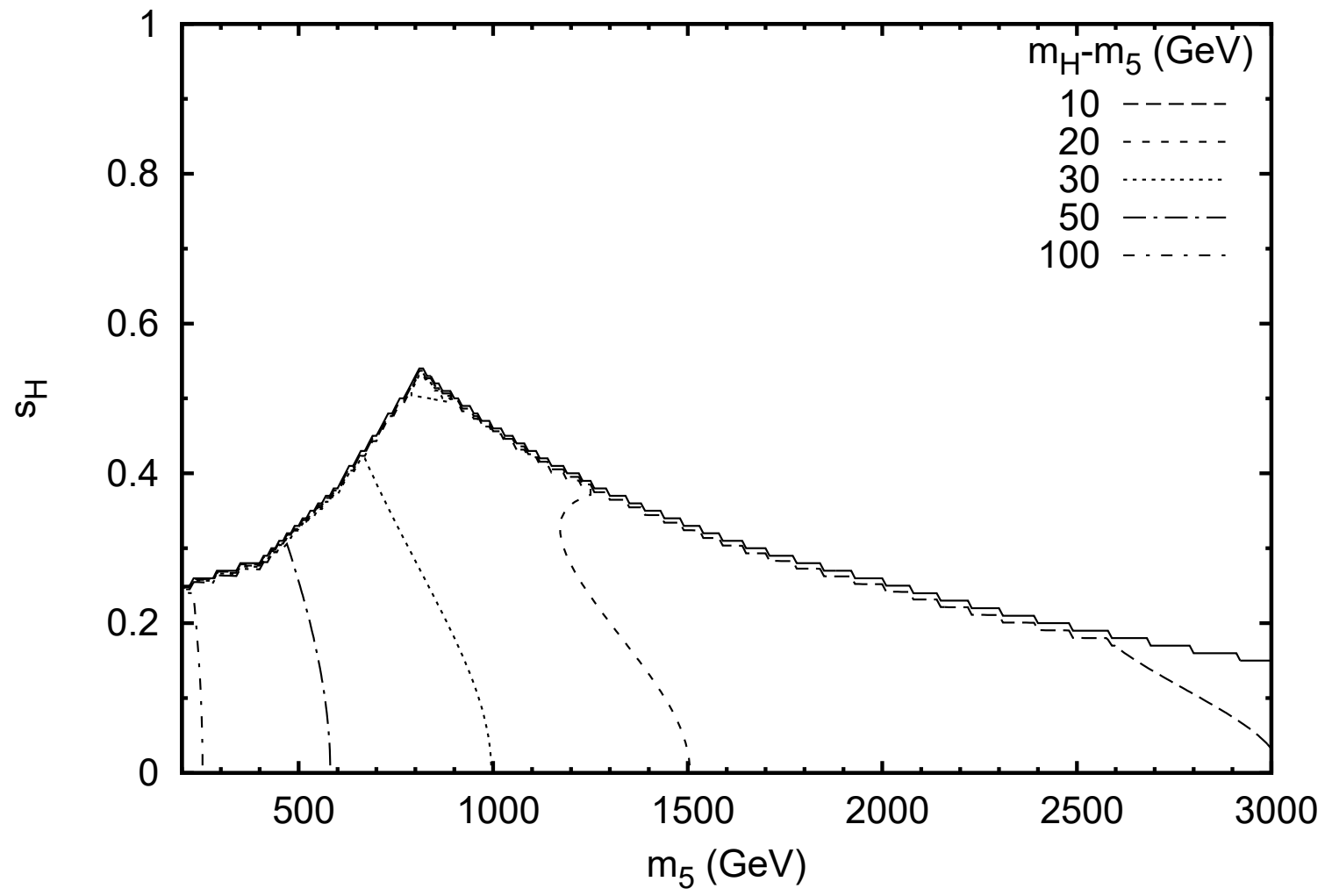

Figure 24: Contours of $m_{H}-m_{5}$ in the H5plane benchmark. $m_{H}-m_{5}$ ranges from $8.9 \mathrm{GeV}$ to $120 \mathrm{GeV}$. 


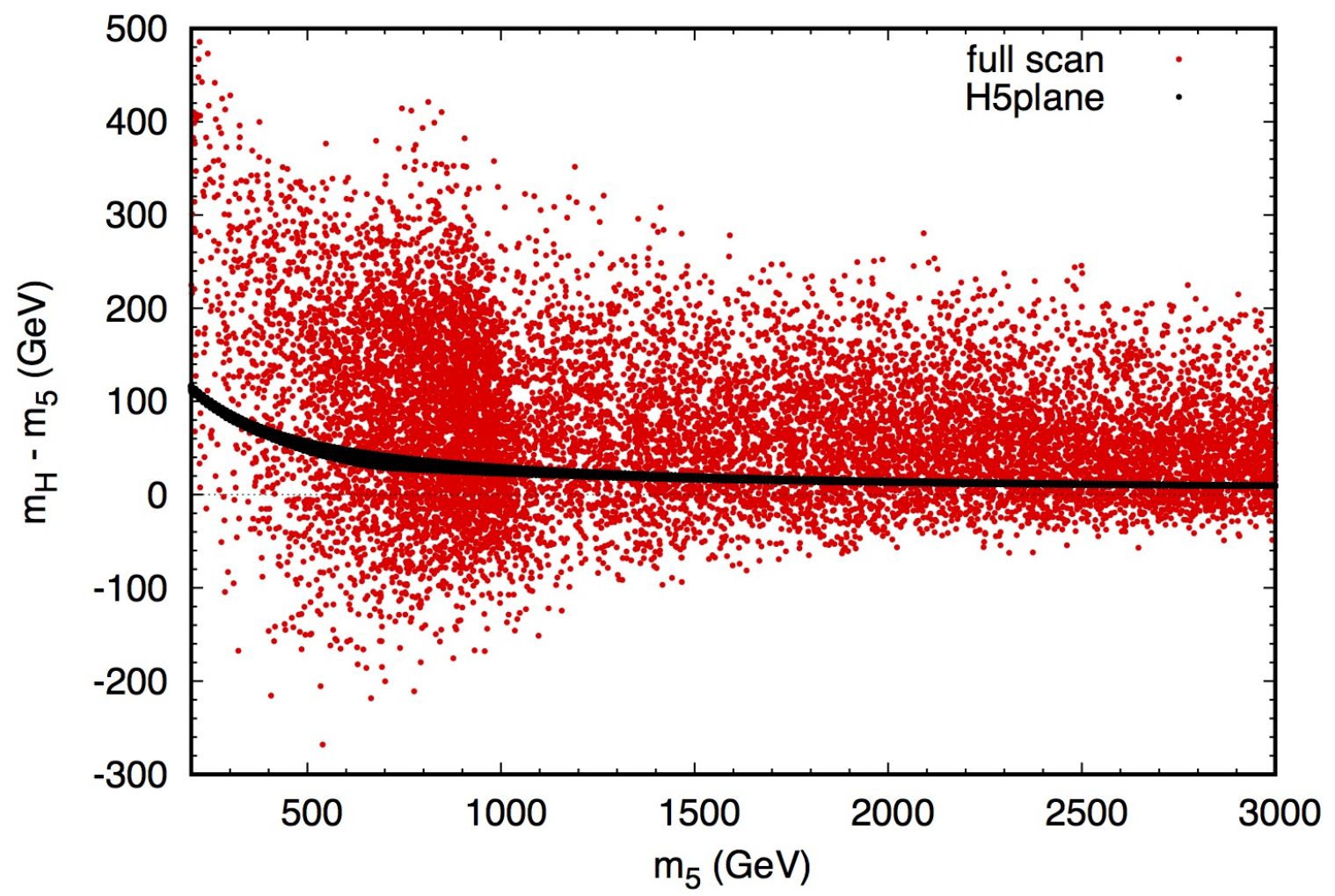

Figure 25: Mass difference $m_{H}-m_{5}$ as a function of $m_{5}$ in the H5plane benchmark (black points) and in a full scan of the GM model parameter space (red points). Indirect constraints from $b \rightarrow s \gamma$ and the $S$ parameter [10] and direct constraints from a CMS search for $H_{5}^{ \pm \pm} \rightarrow W^{ \pm} W^{ \pm}$in vector boson fusion [11] have been applied. 
twice as large as that of $H_{5}^{0}$ for $m_{5}<450 \mathrm{GeV}$. Over the entire H5plane benchmark, the width of $H$ is never less than $89 \%$ of the width of $H_{5}^{0}$.

Therefore we can quantify the $H-H_{5}^{0}$ mass splitting by comparing it to the total width of $H$. We do this in Fig. 28, in which we plot $\left(m_{H}-m_{5}\right) / \Gamma_{\text {tot }}(H)$ over the H5plane benchmark. This ratio varies widely over the benchmark. For low $m_{5}$ and low $s_{H},\left(m_{H}-m_{5}\right) / \Gamma_{\text {tot }}(H)$ is large, which means that the $H$ and $H_{5}^{0}$ resonances are well separated compared to their intrinsic widths. However, there is a sizable region of parameter space in which $\left(m_{H}-m_{5}\right) / \Gamma_{\text {tot }}(H)<1$, which means that the mass splitting is less than the intrinsic width of $H$. In this region of the H5plane benchmark, the total width of $H_{5}^{0}$ is within $10 \%$ of that of $H$. In this case the two resonances overlap significantly and interfere, so that experimental searches for these two states in vector boson fusion with decays to $W^{+} W^{-}$or $Z Z$ must be performed taking into account both resonances and their interference. Interference can be avoided by searching for $H$ produced in gluon fusion, or decaying to $h h$ or $t \bar{t}$, none of which can happen for $H_{5}^{0}$.

\subsection{Decays of $H_{3}$}

The dominant decays of $H_{3}^{0}$ in the H5plane benchmark are to $t \bar{t}, h Z, H_{5}^{0} Z$, and $H_{5}^{ \pm} W^{\mp}$. ( $H_{3}^{0}$ can also decay to two photons; however, $\operatorname{BR}\left(H_{3}^{0} \rightarrow \gamma \gamma\right)$ stays below $1.8 \times 10^{-4}$ over the entire H5plane benchmark.) We plot the branching ratios for these modes in Figs. 29, 30, 31, and 32. The kinematic threshold for $H_{3}^{0} \rightarrow t \bar{t}$ at $m_{3}=2 m_{t}$ occurs at $m_{5}$ just below $300 \mathrm{GeV}$. Once above this threshold, $\mathrm{BR}\left(H_{3}^{0} \rightarrow t \bar{t}\right)$ quickly rises to a maximum of $79 \%$ for $m_{5} \sim 300-400 \mathrm{GeV}$, and then falls with increasing $m_{5}$. The next-largest fermionic decay branching ratio of $H_{3}^{0}$ is to $b \bar{b}$, which is below $1 \%$ over almost all of the H5plane benchmark. The branching ratio of $H_{3}^{0}$ to $h Z$ exhibits complementary behaviour, growing with $m_{5}$ to become the dominant decay mode (>50\%) for $m_{5} \gtrsim 500 \mathrm{GeV}$ and surpassing $90 \%$ branching ratio for $m_{5} \gtrsim 1200 \mathrm{GeV}$. 


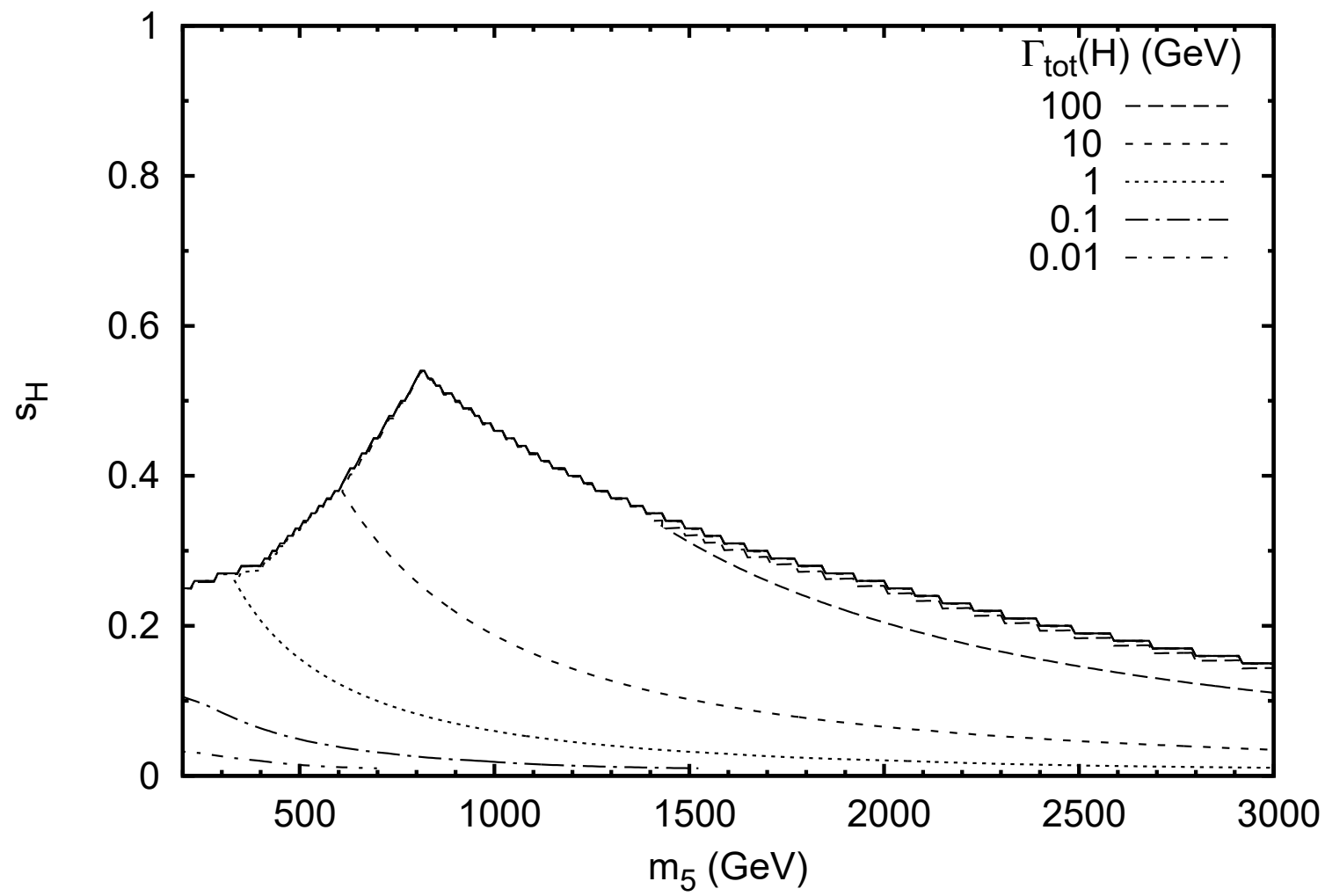

Figure 26: Contours of the total width of $H, \Gamma_{\text {tot }}(H)$, in the H5plane benchmark. $\Gamma_{\text {tot }}(H)$ ranges from $0.0013 \mathrm{GeV}$ to $170 \mathrm{GeV}$. 


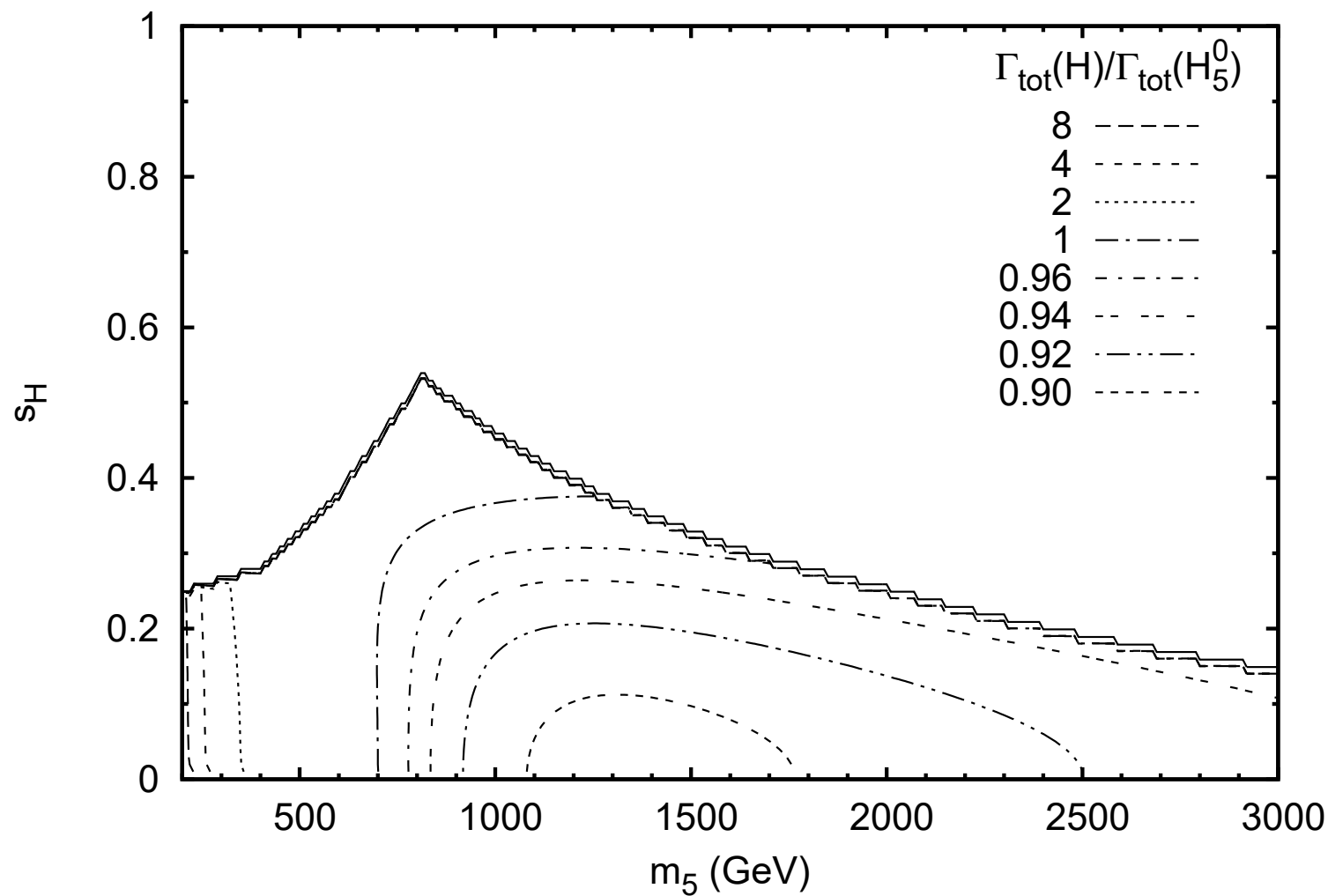

Figure 27: Contours of the ratio $\Gamma_{\text {tot }}(H) / \Gamma_{\text {tot }}\left(H_{5}^{0}\right)$ in the H5plane benchmark. $\Gamma_{\text {tot }}(H) / \Gamma_{\text {tot }}\left(H_{5}^{0}\right)$ ranges from 0.89 to 16 . 


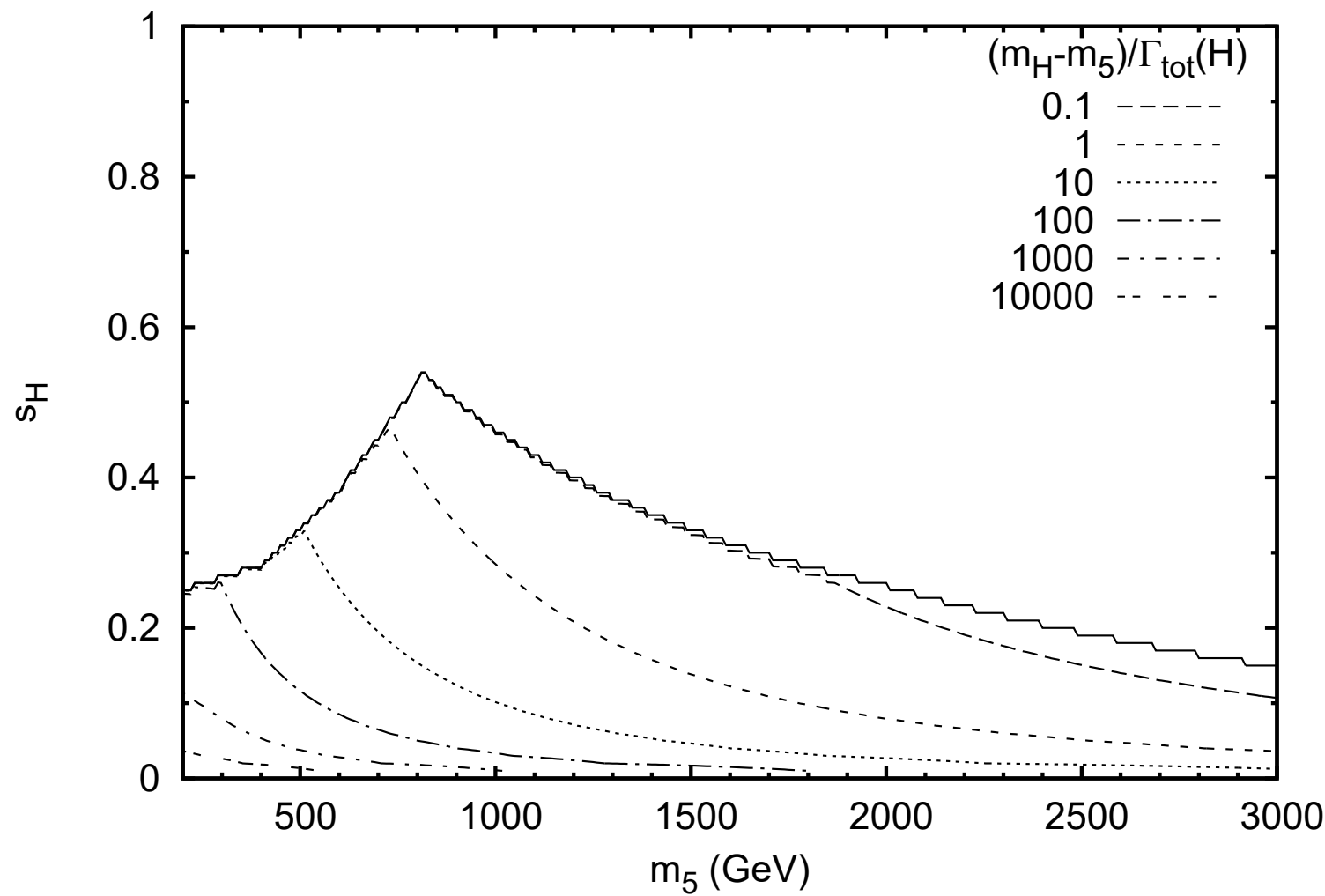

Figure 28: Contours of $\left(m_{H}-m_{5}\right) / \Gamma_{\text {tot }}(H)$ in the H5plane benchmark. $\left(m_{H}-\right.$ $\left.m_{5}\right) / \Gamma_{\text {tot }}(H)$ ranges from 0.054 to 89000 . 


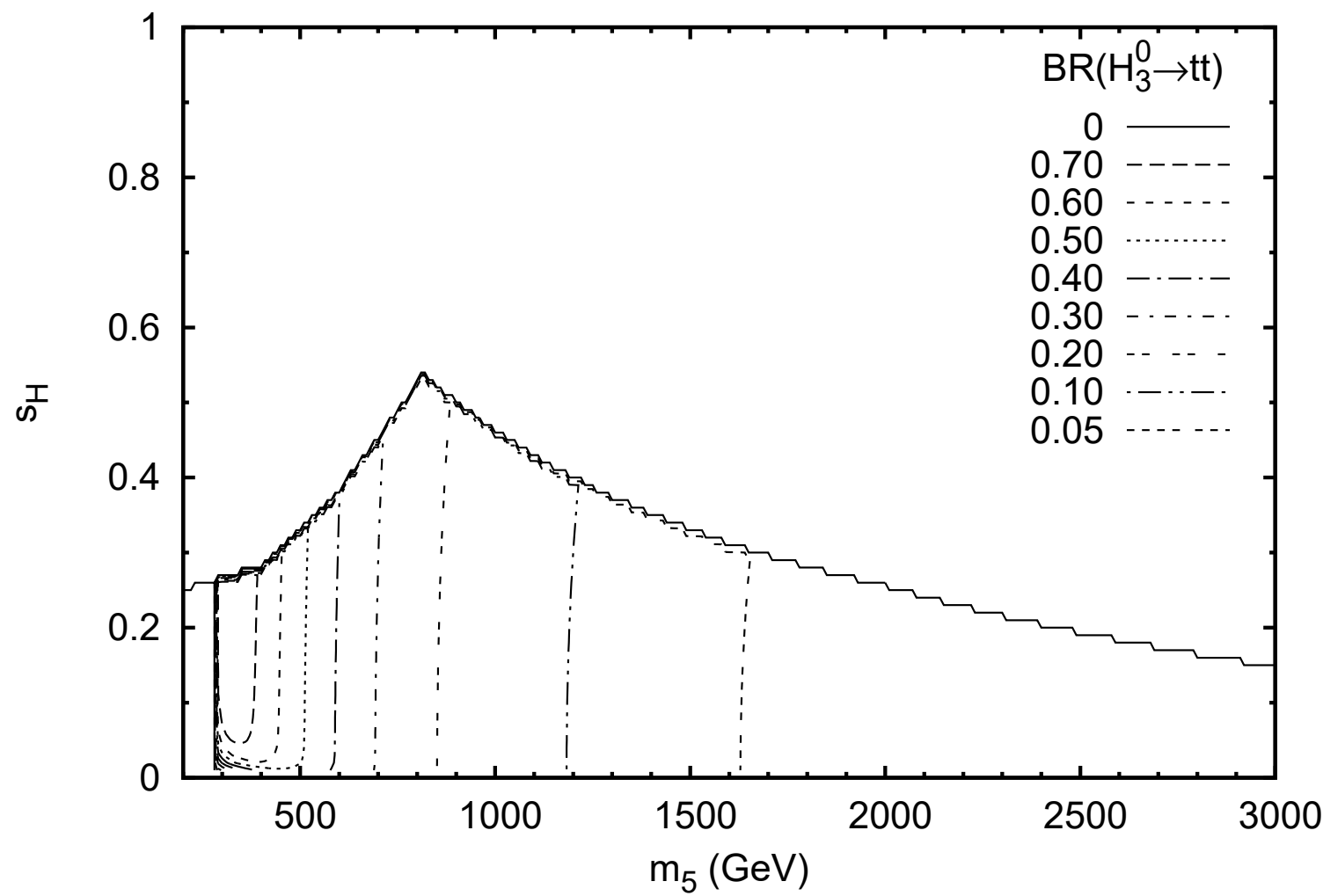

Figure 29: Contours of $\mathrm{BR}\left(H_{3}^{0} \rightarrow t \bar{t}\right)$ in the H5plane benchmark. $\mathrm{BR}\left(H_{3}^{0} \rightarrow t \bar{t}\right)$ ranges from zero to 0.79 . Below the kinematic threshold at $m_{3}=2 m_{t}$, the branching ratio drops to zero because off-shell decays to $t \bar{t}$ are not calculated in GMCALC 1.2.1. $\mathrm{BR}\left(H_{3}^{0} \rightarrow t \bar{t}\right)$ reaches a maximum of 0.79 and falls to 0.013 at $m_{5}=3000 \mathrm{GeV}$. 


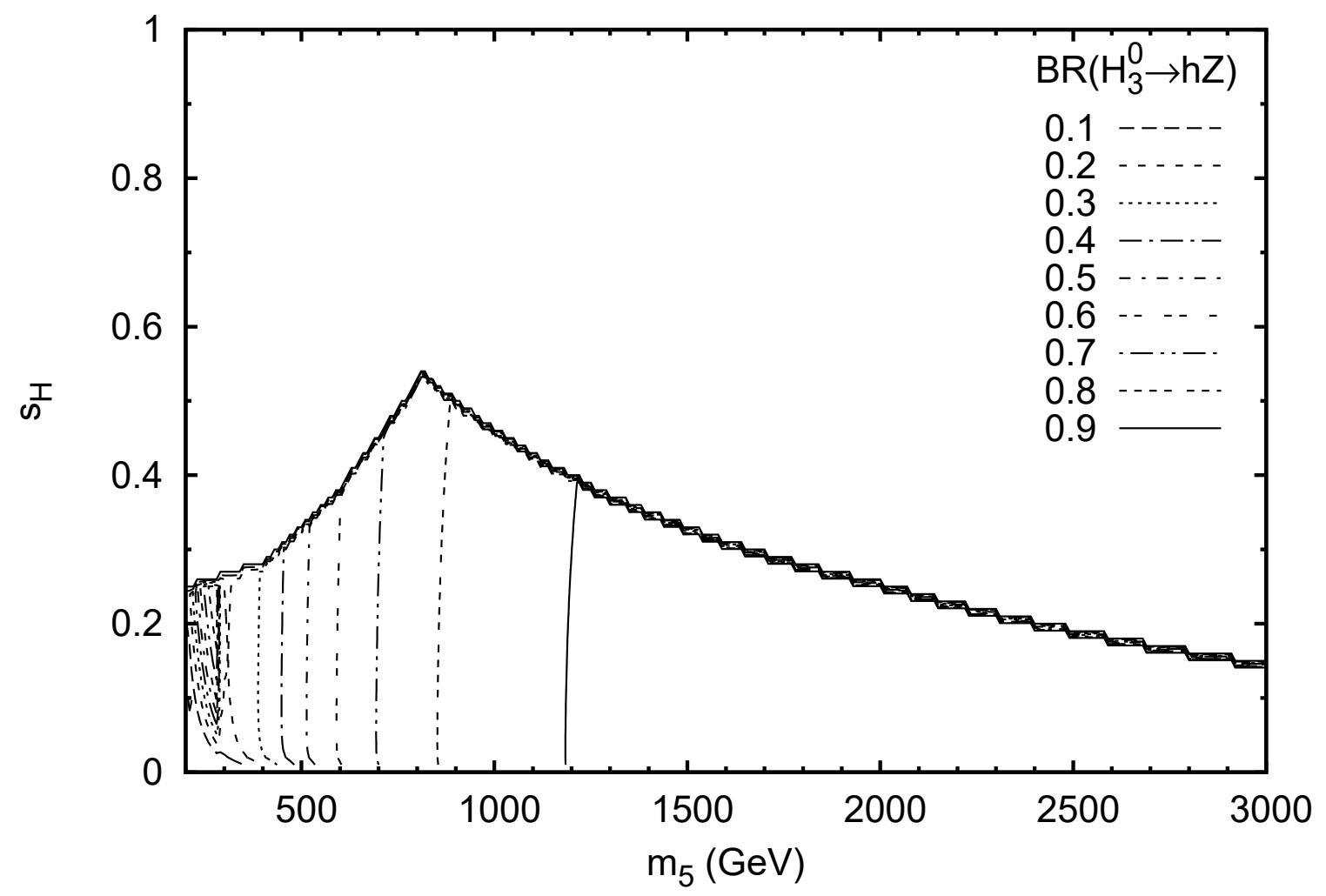

Figure 30: Contours of $\mathrm{BR}\left(H_{3}^{0} \rightarrow h Z\right)$ in the H5plane benchmark. $\mathrm{BR}\left(H_{3}^{0} \rightarrow h Z\right)$ ranges from $2 \times 10^{-4}$ to 0.987 . In the band $m_{5} \in(200 \mathrm{GeV}, 300 \mathrm{GeV})$, the branching ratio increases rapidly, up to nearly 0.9 for $m_{5}=280 \mathrm{GeV}$, before collapsing down to about $0.2 ; \mathrm{BR}\left(H_{3}^{0} \rightarrow h Z\right)$ then rises with increasing $m_{5}$. The sudden drop in $\operatorname{BR}\left(H_{3}^{0} \rightarrow h Z\right)$ is due to crossing the kinematic threshold for $H_{3}^{0} \rightarrow t \bar{t}$. 
The branching ratios of $H_{3}^{0}$ to $H_{5}^{0} Z$ and $H_{5}^{ \pm} W^{\mp}$ (we plot the sum of the branching ratios to $H_{5}^{+} W^{-}$and $H_{5}^{-} W^{+}$) are significant only for very low $m_{5}$, below the kinematic threshold for the $t \bar{t}$ decay. For these low masses, the branching ratios of these modes can be quite large, reaching respective values of $85 \%$ and $82 \%$ in our calculation, in slightly different areas of parameter space. However, these numbers should be treated with caution because the implementation in GMCALC 1.2.1 of scalar decays to scalar plus vector at and below the kinematic threshold is still rather primitive. At $m_{5}=200 \mathrm{GeV}$, the mass splitting between $H_{3}$ and $H_{5}$ in the H5plane benchmark is $84 \mathrm{GeV}$, so that the on-shell decay $H_{3}^{0} \rightarrow H_{5}^{ \pm} W^{\mp}$ is barely kinematically allowed, while $H_{3}^{0} \rightarrow H_{5}^{0} Z$ is off-shell. As $m_{5}$ increases, the mass splitting decreases, and $H_{3}^{0} \rightarrow H_{5}^{ \pm} W^{\mp}$ goes off-shell at $m_{5} \simeq 210 \mathrm{GeV}$. Above threshold, GMCALC 1.2.1 computes these decay widths using the two-body on-shell decay formula, while below threshold the computation takes into account the offshellness of the vector boson only. This is a reasonable approximation at $m_{5} \sim 200 \mathrm{GeV}$ where the $H_{5}$ scalars are very narrow; however, the transition from the on-shell to off-shell decay widths is not smooth. The handling of this transition, along with off-shell decays of $H_{3}^{0} \rightarrow t \bar{t}$, should be improved if detailed predictions for the $H_{3}^{0}$ branching ratios for $m_{5} \lesssim 280 \mathrm{GeV}$ are needed. The branching ratios for $H_{3}^{0}$ to $H_{5}^{0} Z$ and $H_{5}^{ \pm} W^{\mp}$ fall below $1 \%$ for $m_{5} \gtrsim 500 \mathrm{GeV}$.

The dominant decays of $H_{3}^{+}$in the H5plane benchmark are to $t \bar{b}, h W^{+}, H_{5}^{0} W^{+}$, $H_{5}^{+} Z$, and $H_{5}^{++} W^{-}$. We plot the branching ratios for these modes in Figs. 33, 34, 35,36 , and 37 . The decay to $t \bar{b}$ dominates at low $m_{5}$, reaching a maximum of more than $95 \%$ for $m_{5} \sim 250 \mathrm{GeV}$. This branching ratio falls with increasing $m_{5}$ and is supplanted by the decay to $h W^{+}$. The branching ratio for $H_{3}^{+} \rightarrow h W^{+}$becomes dominant (> $50 \%$ ) for $m_{5} \gtrsim 500 \mathrm{GeV}$ and surpasses $90 \%$ when $m_{5} \gtrsim 1200 \mathrm{GeV}$.

The branching ratios of $H_{3}^{+}$to $H_{5}^{0} W^{+}, H_{5}^{+} Z$, and $H_{5}^{++} W^{-}$are significant only for very low values of both $m_{5}$ and $s_{H}$ within the H5plane benchmark. In this corner 


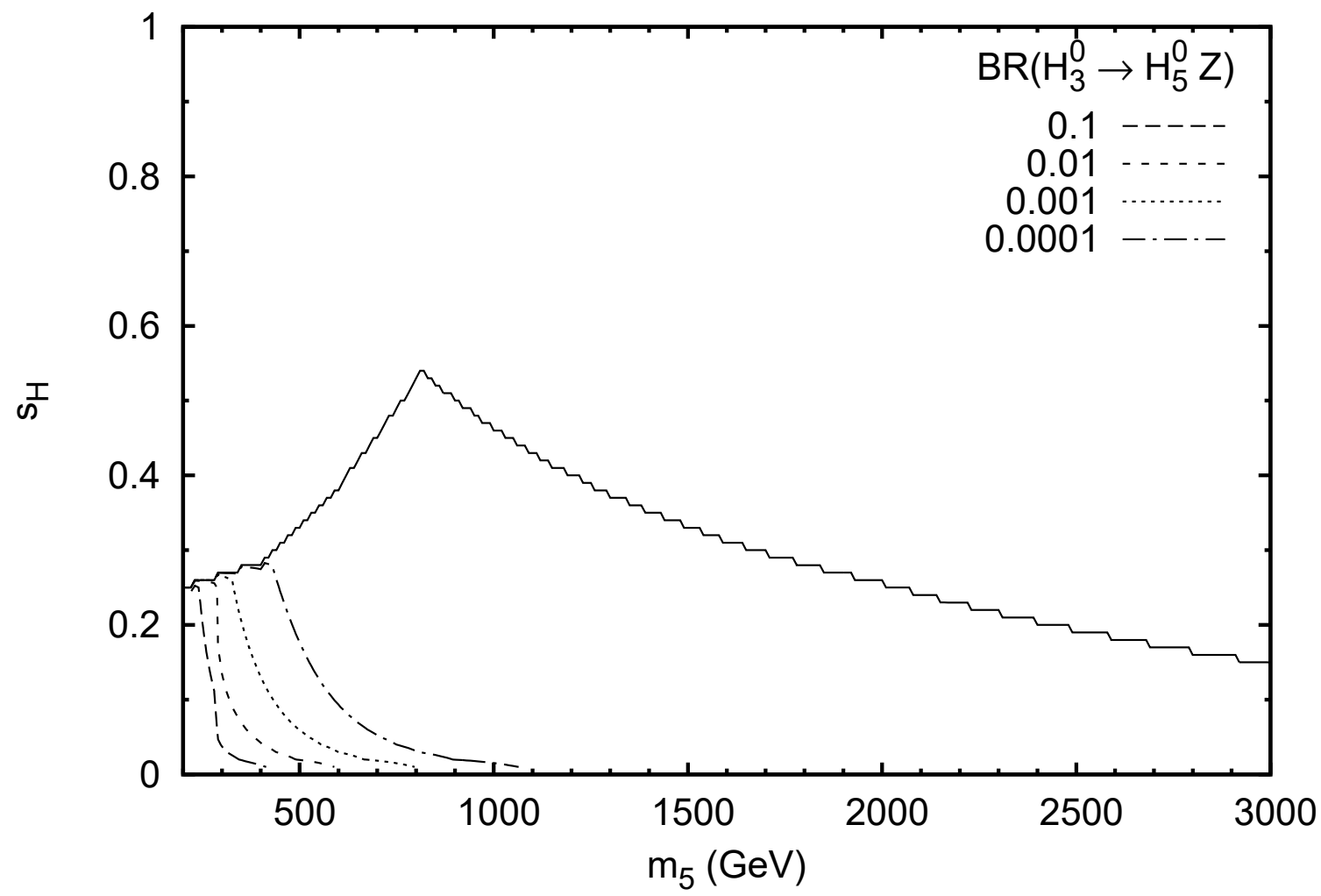

Figure 31: Contours of $\mathrm{BR}\left(H_{3}^{0} \rightarrow H_{5}^{0} Z\right)$ in the H5plane benchmark. See text for further discussion. 


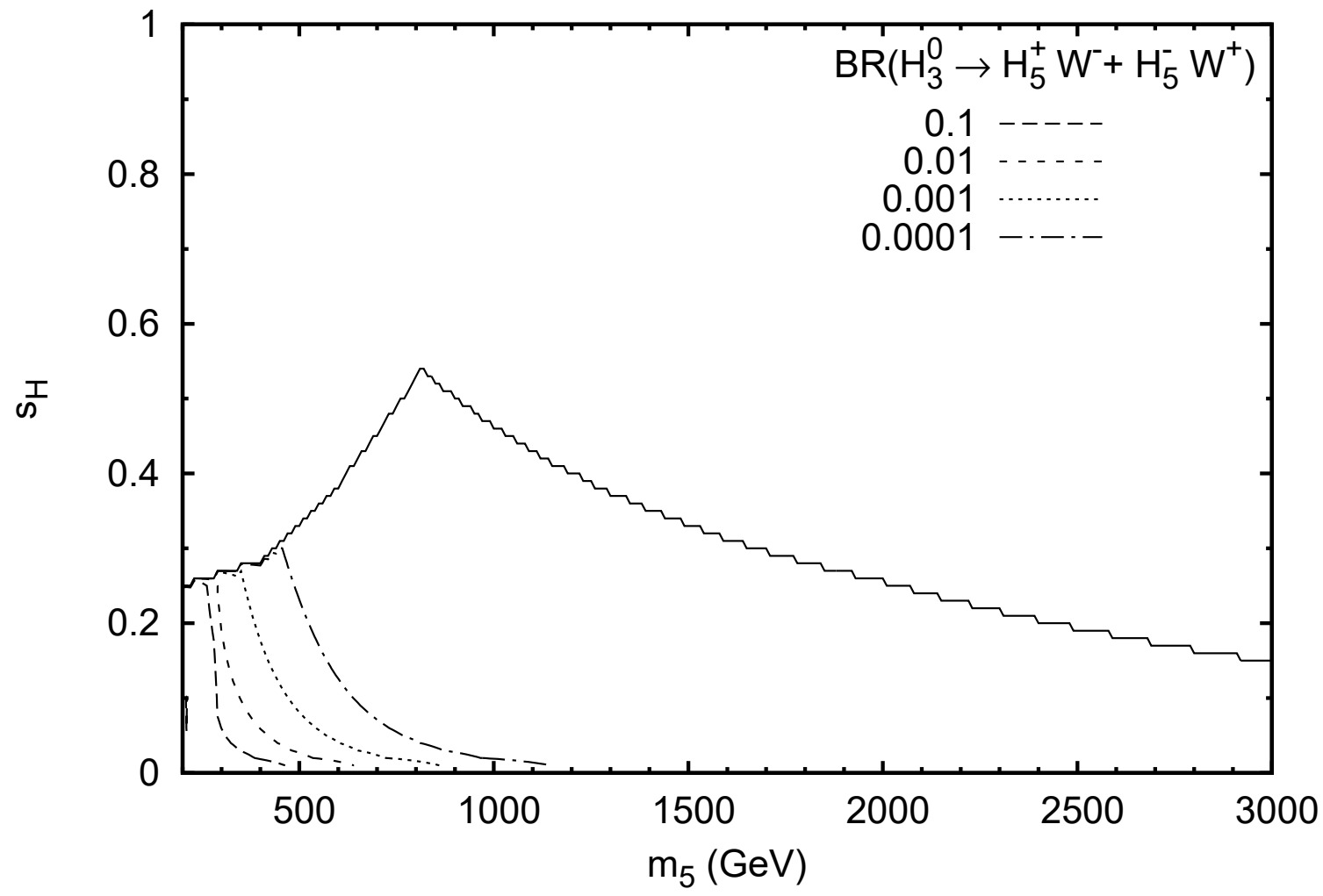

Figure 32: Contours of $\mathrm{BR}\left(H_{3}^{0} \rightarrow H_{5}^{+} W^{-}+H_{5}^{-} W^{+}\right)$in the H5plane benchmark. See text for further discussion. 


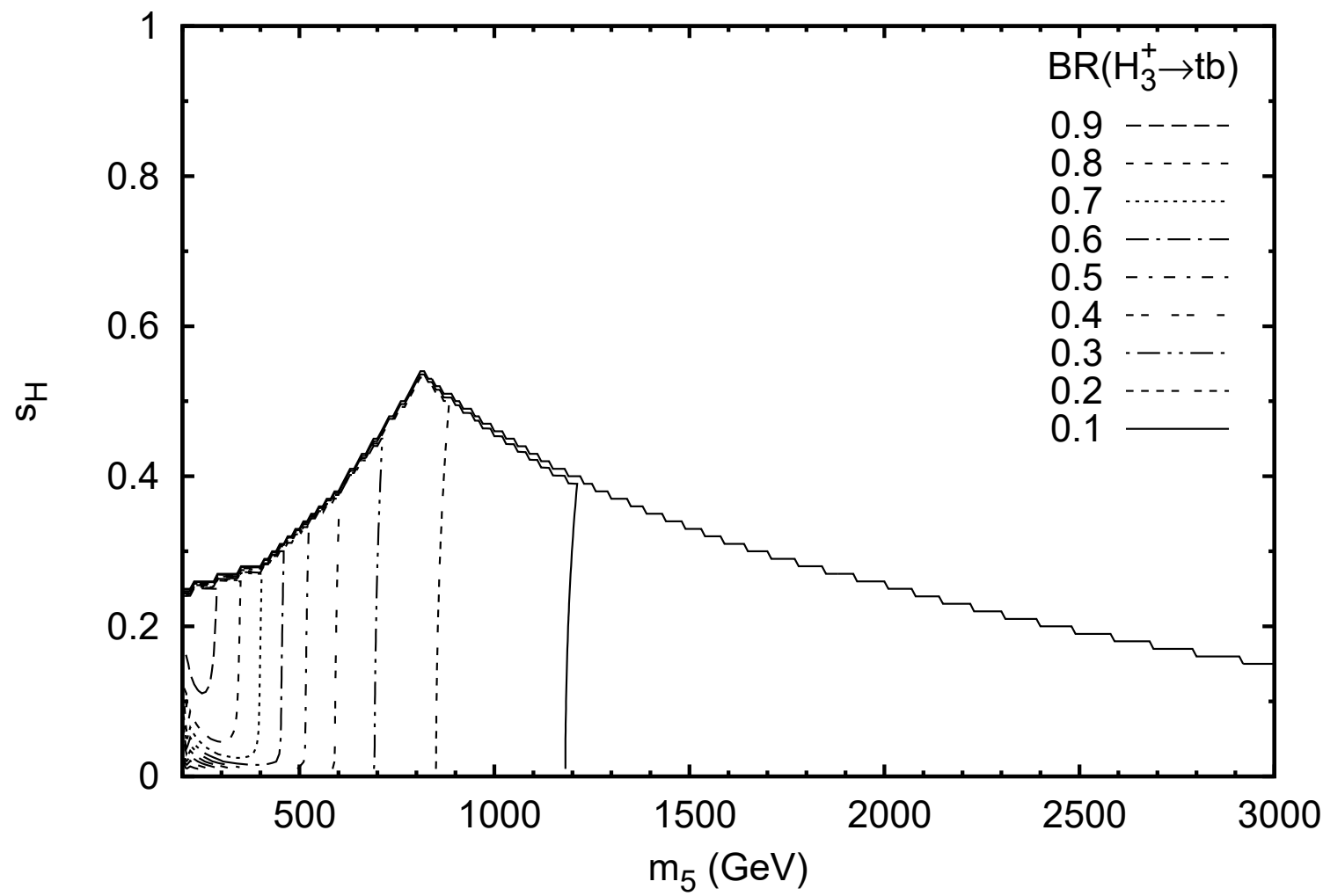

Figure 33: Contours of $\mathrm{BR}\left(H_{3}^{+} \rightarrow t \bar{b}\right)$ in the H5plane benchmark. $\mathrm{BR}\left(H_{3}^{+} \rightarrow t \bar{b}\right)$ ranges from 0.013 to 0.964 . 


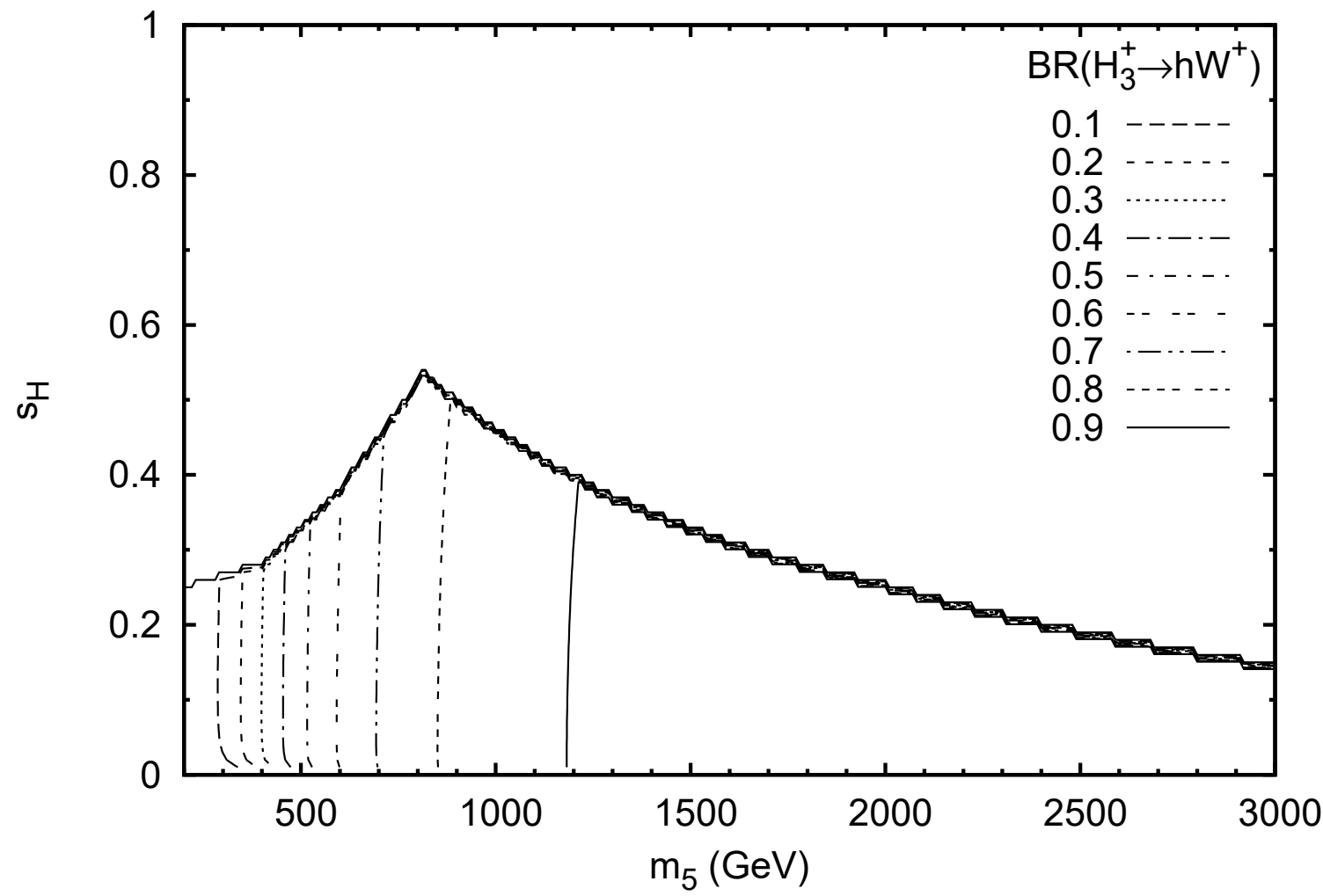

Figure 34: Contours of $\mathrm{BR}\left(H_{3}^{+} \rightarrow h W^{+}\right)$in the H5plane benchmark. $\mathrm{BR}\left(H_{3}^{+} \rightarrow\right.$ $h W^{+}$) ranges from $3 \times 10^{-4}$ to 0.987 . 


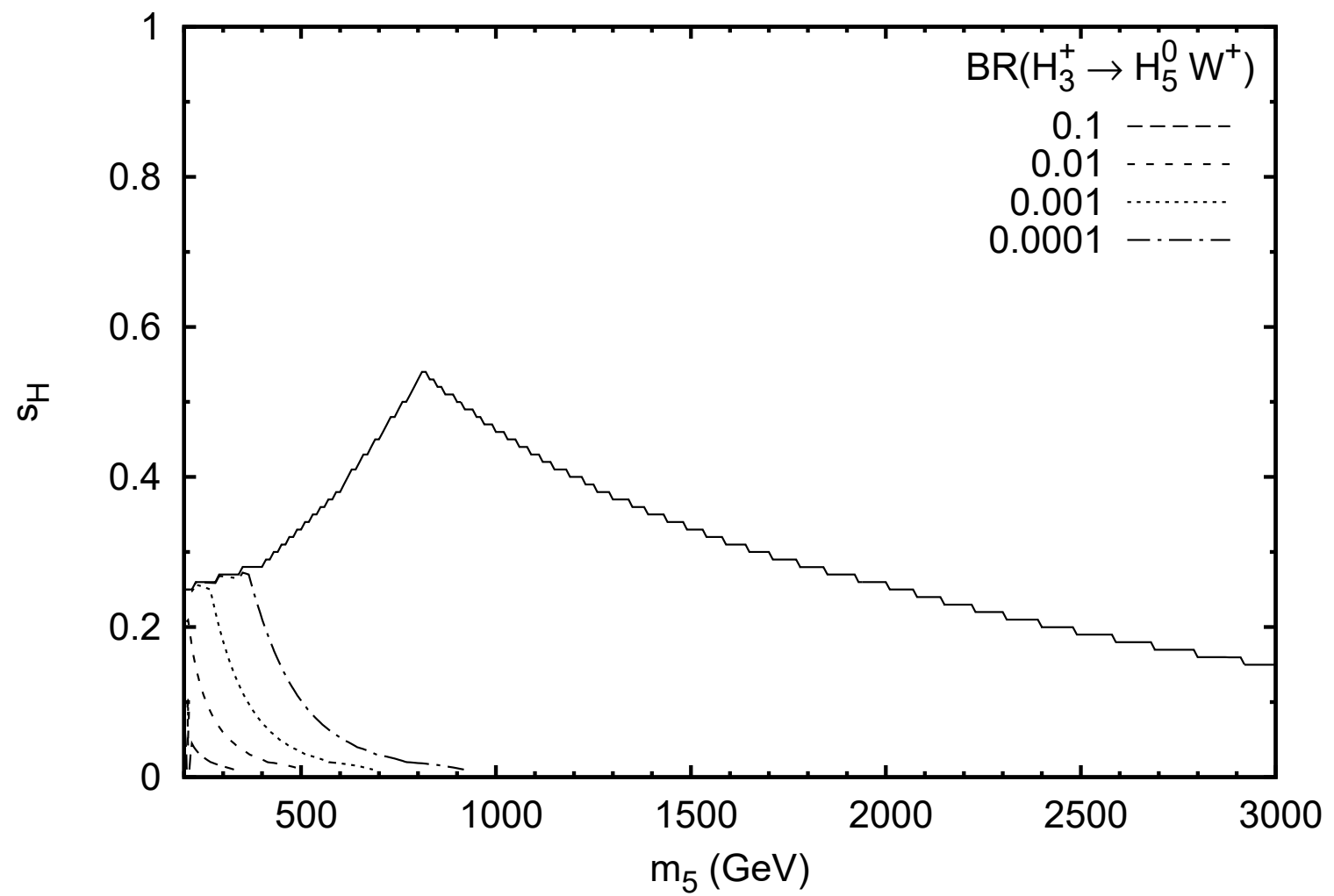

Figure 35: Contours of $\mathrm{BR}\left(H_{3}^{+} \rightarrow H_{5}^{0} W^{+}\right)$in the H5plane benchmark. See text for further discussion.

of parameter space, the branching ratios of these modes can be significant, reaching maxima of $25 \%, 79 \%$, and $49 \%$, respectively, in slightly different regions of parameter space. Again, though, these numbers should be treated with caution because the decays of $H_{3}^{+}$to $H_{5} V$ face the same issues with the transition from on-shell to offshell as the decays of $H_{3}^{0}$ to $H_{5} V$. All three of these branching ratios quickly fall below the $1 \%$ level for $m_{5} \gtrsim 500 \mathrm{GeV}$. These decay modes also decline quickly with increasing $s_{H}$, due to an increase in the partial width for $H_{3}^{+} \rightarrow t \bar{b}$ with increasing $s_{H}$.

Finally, we plot the total widths of $H_{3}^{0}$ and $H_{3}^{+}$in Figs. 38 and 39. They both remain quite small over the entire allowable region: although they do increase with increasing $s_{H}$ and $m_{5}$, the width-to-mass ratio $\Gamma_{\text {tot }}\left(H_{3}\right) / m_{3}$ never rises above $8 \%$ for either $H_{3}^{0}$ or $H_{3}^{+}$. 


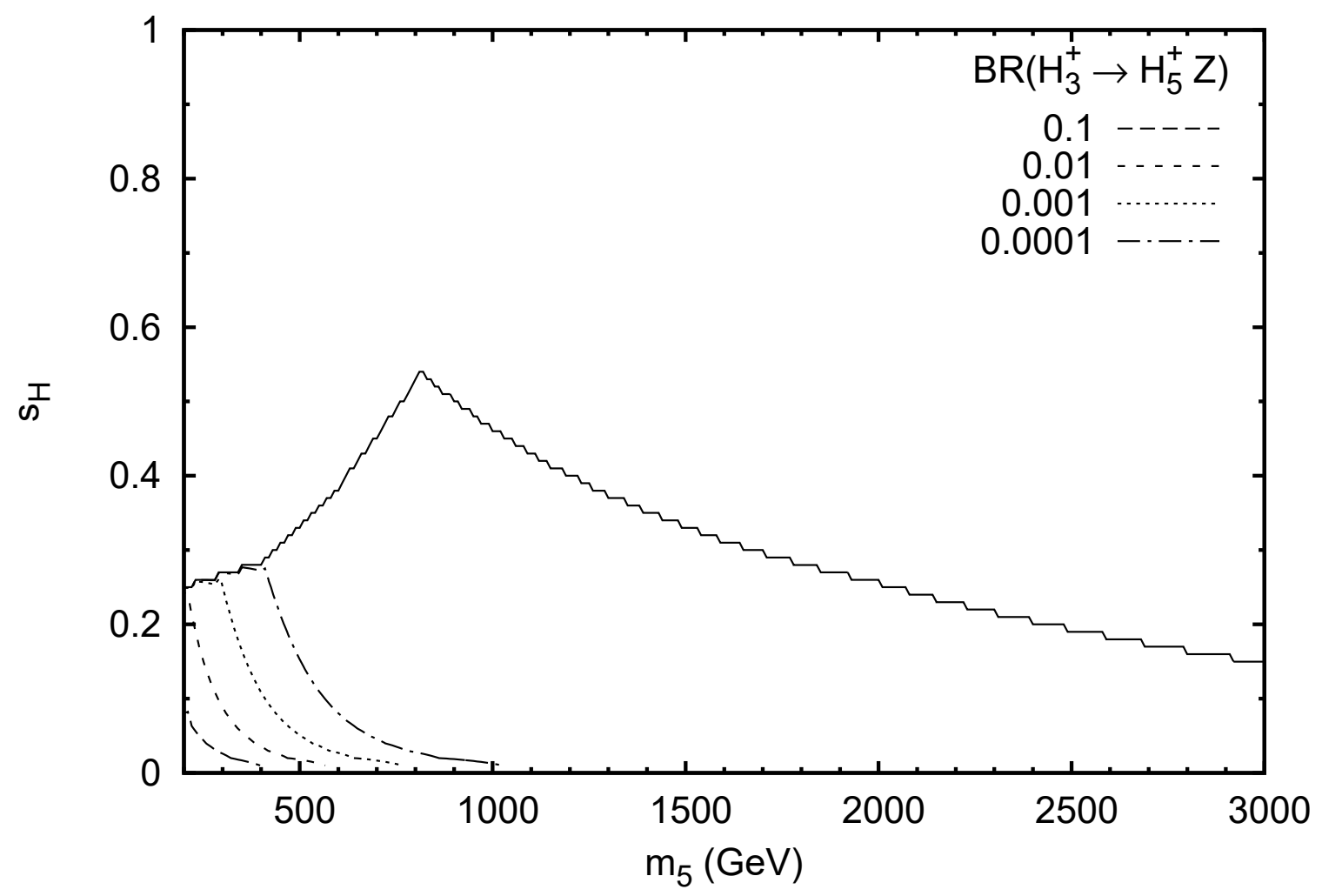

Figure 36: Contours of $\mathrm{BR}\left(H_{3}^{+} \rightarrow H_{5}^{+} Z\right)$ in the H5plane benchmark. See text for further discussion. 


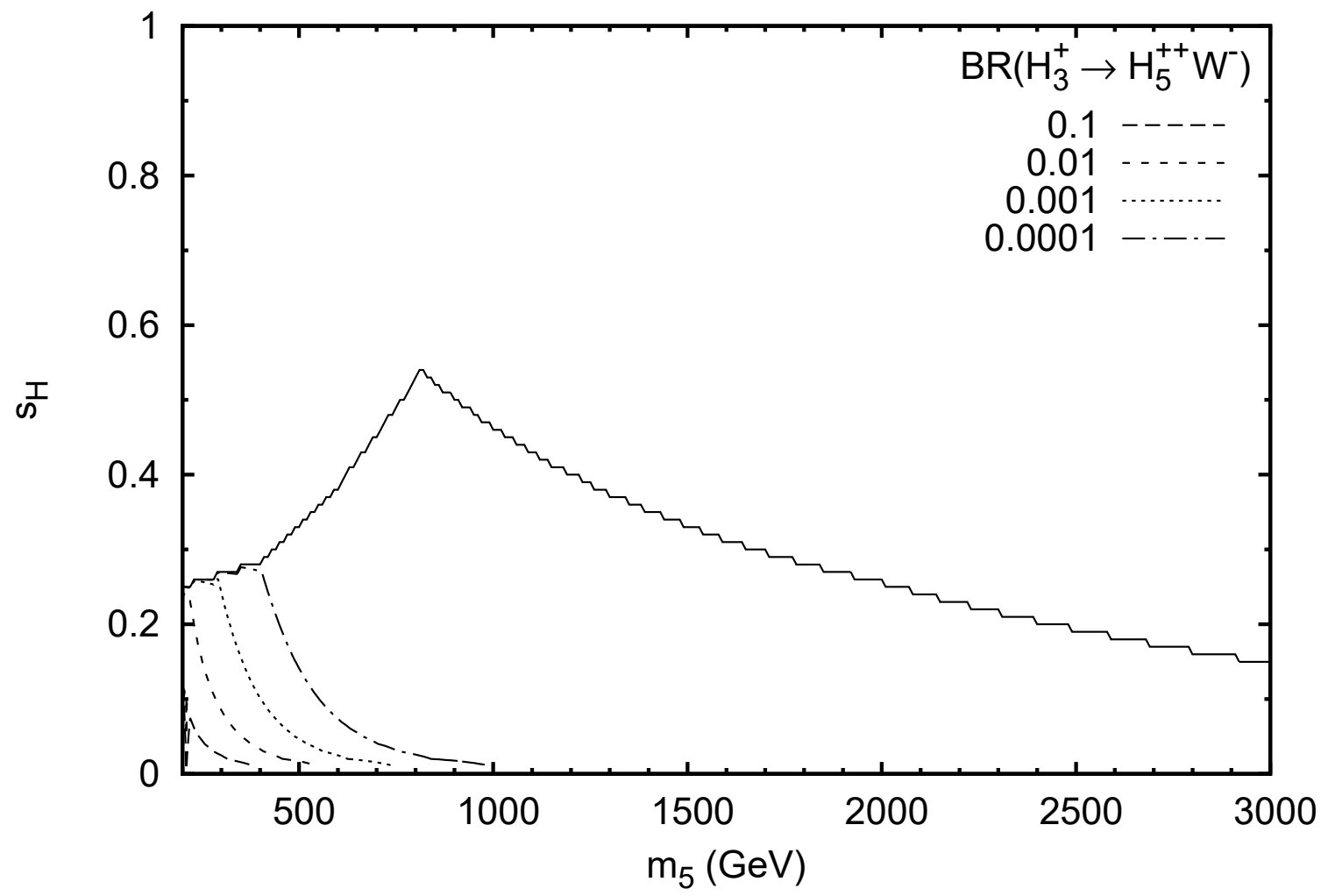

Figure 37: Contours of $\mathrm{BR}\left(H_{3}^{+} \rightarrow H_{5}^{++} W^{-}\right)$in the H5plane benchmark. See text for further discussion. 


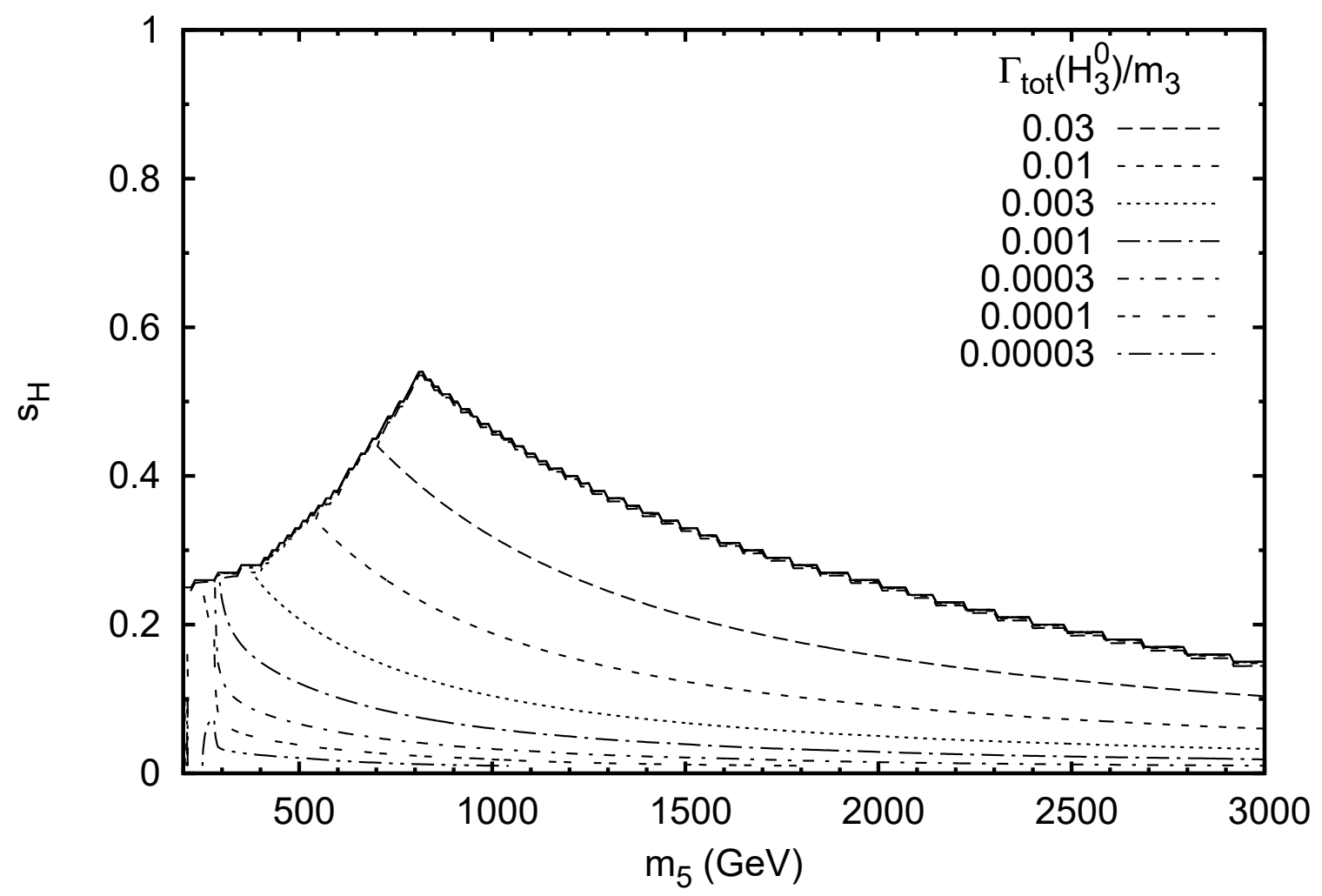

Figure 38: Contours of $\Gamma_{\text {tot }}\left(H_{3}^{0}\right) / m_{3}$ in the H5plane benchmark. $\Gamma_{\text {tot }}\left(H_{3}^{0}\right) / m_{3}$ ranges from $6.6 \times 10^{-6}$ to 0.077 . 


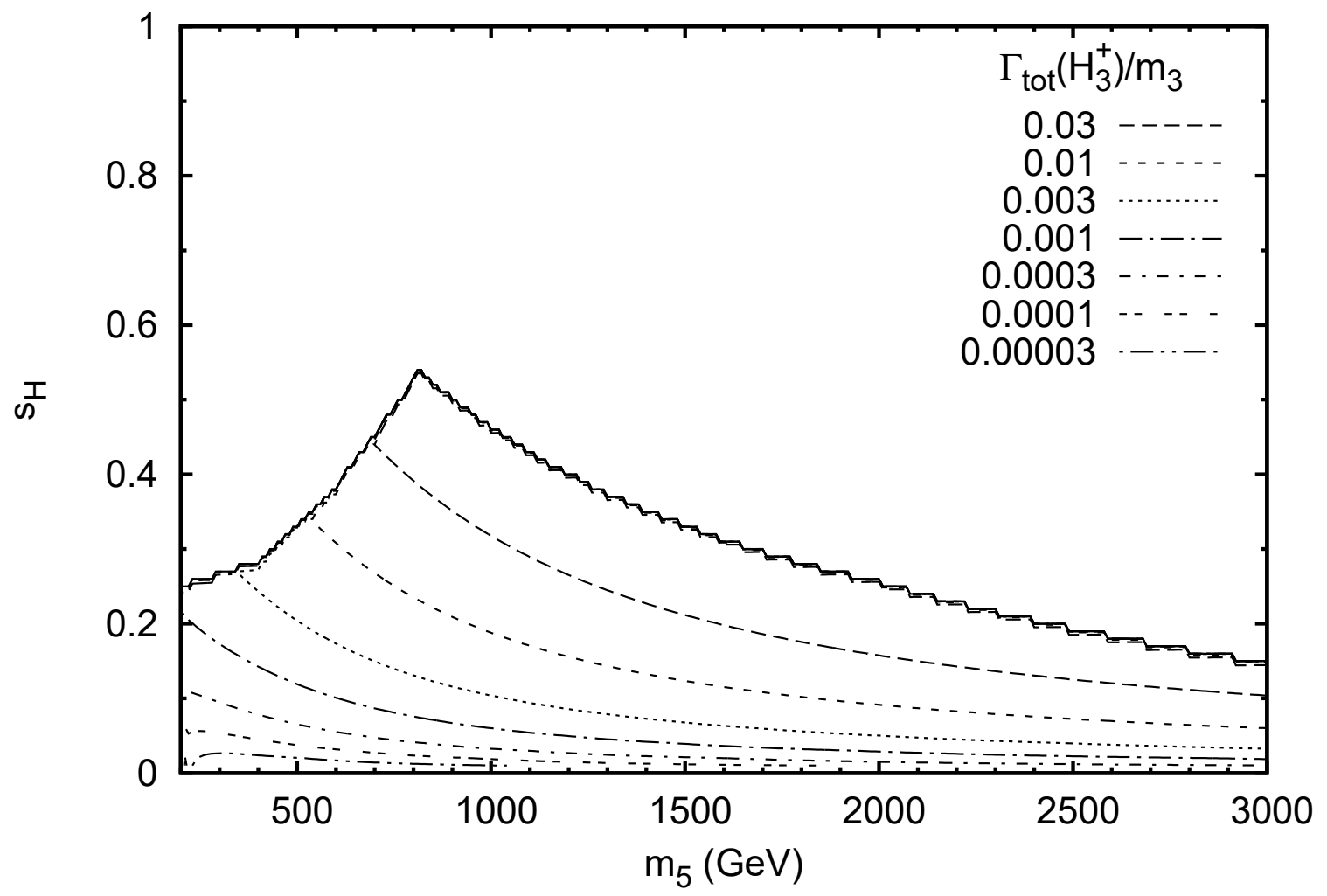

Figure 39: Contours of $\Gamma_{\text {tot }}\left(H_{3}^{+}\right) / m_{3}$ in the H5plane benchmark. $\Gamma_{\text {tot }}\left(H_{3}^{+}\right) / m_{3}$ ranges from $6.2 \times 10^{-6}$ to 0.077 . 
While the calculations used for our predictions are sufficiently accurate for our purposes, we have seen that some of the decay processes, i.e. decays to $t \bar{t}$ (Figs. 23 and 29) and decays to $H_{5}$ and a vector boson (Figs. 31, 32, 35, 36, 37), show signs of discontinuity and do not fully capture expected offshell behaviour in the lowmass range in GMCALC 1.2.1. From a computational perspective, the decays of the form $\mathrm{H}_{3} \rightarrow \mathrm{H}_{5} \mathrm{~V}$ use an algebraic formula for offshell decay, rather than using a more accurate integration routine, and switch discontinuously between computing onshell two-body decay, and decay with an offshell $V$, at the energetic threshold. The decays to $t \bar{t}$, meanwhile, are onshell only, and don't account for offshell behaviour at all. These choices were made because fully accounting for offshell behaviour requires numerical integration, which is computationally expensive. To remedy this, we have been implementing procedures to allow GMCALC to compute offshell decay processes more efficiently and accurately; some of these procedures have already been included in GMCALC 1.4.0. We will discuss these improvements to the code in the next section. 


\section{Offshell decay algorithms}

For most of the decay processes in GMCALC, the decaying particle is far heavier than its decay products. However, some decay processes, such as the decay of a scalar particle to two massive vector bosons (e.g. $h \rightarrow W^{+} W^{-}, H_{5} \rightarrow Z Z$, etc.) take into account the effects of offshell decays. Because offshell particles are not of fixed mass, the calculation of the decay rates involves an integral, which is solved numerically. Since the GMCALC code does not take e.g. electroweak loop corrections into account, our predictions will likely be off by a few percent from the true theoretical value (see e.g. Table 1 of Ref. [21]); as such, so long as the error in our numerical calculations is less than the error due to these unaccounted-for corrections, we can be assured that we have made a reasonable estimate. In GMCALC 1.2.1, the offshell integration procedure is implemented using rectangular integration, which, while accurate, involves a time-consuming number of steps. In this section we will discuss a more efficient integration algorithm that we have incorporated into GMCALC 1.4.0, as well as some decay processes which we are planning to implement to ensure greater accuracy when the scalar particles involved are of low mass.

\section{1 $\quad H_{i} \rightarrow V_{1}^{*} V_{2}^{*}$ integration routine}

The integral to be computed for the $H_{i} \rightarrow V_{1}^{*} V_{2}^{*}$ offshell decay is

$$
\begin{array}{rl}
\Gamma\left(H_{i} \rightarrow V_{1}^{*} V_{2}^{*}\right)=\frac{1}{\pi^{2}} \int_{0}^{m_{H_{i}}^{2}} & d Q_{1}^{2} \int_{0}^{\left(m_{H_{i}}-Q_{1}\right)^{2}} d Q_{2}^{2} \\
\times & \frac{Q_{1}^{2} \Gamma_{V_{1}} / M_{V_{1}}}{\left(Q_{1}^{2}-M_{V_{1}}^{2}\right)^{2}+M_{V_{1}}^{2} \Gamma_{V_{1}}^{2}} \\
& \times \frac{Q_{2}^{2} \Gamma_{V_{2}} / M_{V_{2}}}{\left(Q_{2}^{2}-M_{V_{2}}^{2}\right)^{2}+M_{V_{2}}^{2} \Gamma_{V_{2}}^{2}} \Gamma^{H_{i} V_{1} V_{2}}\left(Q_{1}^{2}, Q_{2}^{2}\right),
\end{array}
$$

where $\Gamma_{V_{i}}$ is the measured total width of gauge boson $V_{i}, Q_{i}^{2}$ is the square of the four-momentum of $V_{i}$, and $\Gamma^{H_{i} V_{1} V_{2}}\left(Q_{1}^{2}, Q_{2}^{2}\right)$ is the on-shell decay width for $H_{i} \rightarrow V_{1} V_{2}$ 
with the squared-masses of the gauge bosons $V_{1}$ and $V_{2}$ replaced by $Q_{1}^{2}$ and $Q_{2}^{2}$. The formula for $\Gamma^{H_{i} V_{1} V_{2}}\left(Q_{1}^{2}, Q_{2}^{2}\right)$ is

$$
\Gamma^{H_{i} V_{1} V_{2}}\left(Q_{1}^{2}, Q_{2}^{2}\right)=S_{V} \frac{\left|g_{H_{i} V_{1} V_{2}}\right|^{2} m_{H_{i}}^{3}}{64 \pi Q_{1}^{2} Q_{2}^{2}}\left[1-2 k_{1}-2 k_{2}+10 k_{1} k_{2}+k_{1}^{2}+k_{2}^{2}\right] \sqrt{\lambda\left(k_{1}, k_{2}\right)},
$$

where $S_{V}$ is a symmetry factor which is 1 if $V_{1}$ and $V_{2}$ are distinct bosons and is $1 / 2$ if $V_{1}$ and $V_{2}$ are identical, $g_{H_{i} V_{1} V_{2}}$ is the coupling constant between the scalar $H_{i}$ and the massive vector bosons $V_{1}$ and $V_{2}$, and $k_{1}=Q_{1}^{2} / m_{H_{i}}^{2}$ and $k_{2}=Q_{2}^{2} / m_{H_{i}}^{2}$. The function $\lambda$ is given by

$$
\lambda(x, y)=(1-x-y)^{2}-4 x y .
$$

Making the change of variables from $Q_{i}^{2}$ to $\rho_{i}$, where

$$
\rho_{i}=\frac{1}{\pi} \tan ^{-1}\left[\frac{Q_{i}^{2}-M_{V_{i}}^{2}}{M_{V_{i}} \Gamma_{V_{i}}}\right]
$$

simplifies the integrand and flattens out the distribution (in its initial form, the integrand is sharply peaked at $Q_{i}=M_{V_{i}}$ ). As such, if $m_{H_{i}}>M_{V_{1}}+M_{V_{2}}$, the integrand is much better behaved when we integrate with respect to $\rho_{i}$.

By examining the behaviour of the integrands for the $H_{i} \rightarrow V_{1} V_{2}$ for various values of $m_{H_{i}}$ (see Figs. 40 to 45 for contour plots for the decay to $W^{+} W^{-}$), we were able to determine the circumstances under which to choose $Q_{i}$ or $\rho_{i}$ as the variables of integration. As mentioned above, $\rho_{i}$ produces a much more uniform integrand at $m_{H_{i}}>M_{V_{1}}+M_{V_{2}}$ than $Q_{i}$ does, so we chose to integrate with respect to $\rho_{i}$ when $m_{H_{i}}$ is sufficiently large. However, for $m_{H_{i}}<M_{V_{1}}+M_{V_{2}}$, the integration region for $\rho_{i}$ becomes quite oddly shaped, only taking up a fraction of the (large, rectangular) space that it extends into. The shape of this region, combined with the fact that the integrand is largest in the small region near the point $(-0.5,-0.5)$, means that many multidimensional integration routines will fail to accurately calculate the 


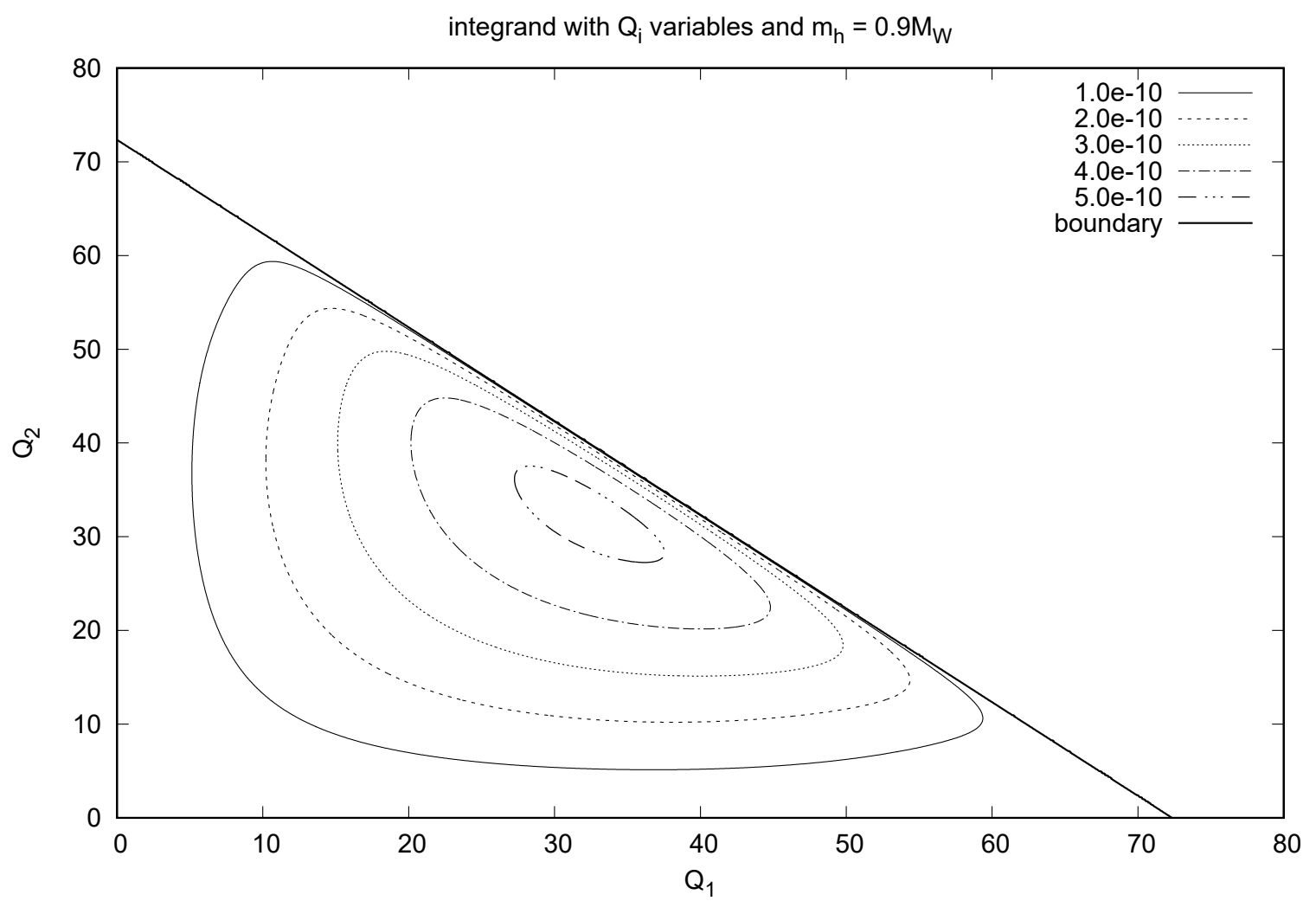

Figure 40: Contours of the integrand with respect to $Q_{i}$ for $H_{i} \rightarrow W^{+} W^{-}$with $m_{H_{i}}=0.9 M_{W} \cdot Q_{1}$ and $Q_{2}$ have units of $\mathrm{GeV}$, and the integrand has units of $[\mathrm{GeV}]^{-1}$. For $m_{H_{i}}<M_{W}$, the integrand is fairly well-behaved.

integral, if they sample insufficiently many points. As such, we decided that when $m_{H_{i}}<M_{V_{1}}+M_{V_{2}}$, one should use $Q_{i}$ rather than $\rho_{i}$ as the variable of integration.

Once we had determined the variables with respect to which we would be integrating, we endeavoured to determine which integration routines would enable us to compute most efficiently while still retaining the desired level of accuracy. For future reference, when we calculate the relative error in some quantity $X$ under some given new calculation routine, $X_{\text {new }}$, as compared to the results of some previous routine, $X_{\text {prev }}$, we are essentially calculating the following:

$$
\frac{X_{\text {new }}-X_{\text {prev }}}{X_{\text {prev }}}
$$

When we were testing the accuracy of the integration routines for the offshell decay 


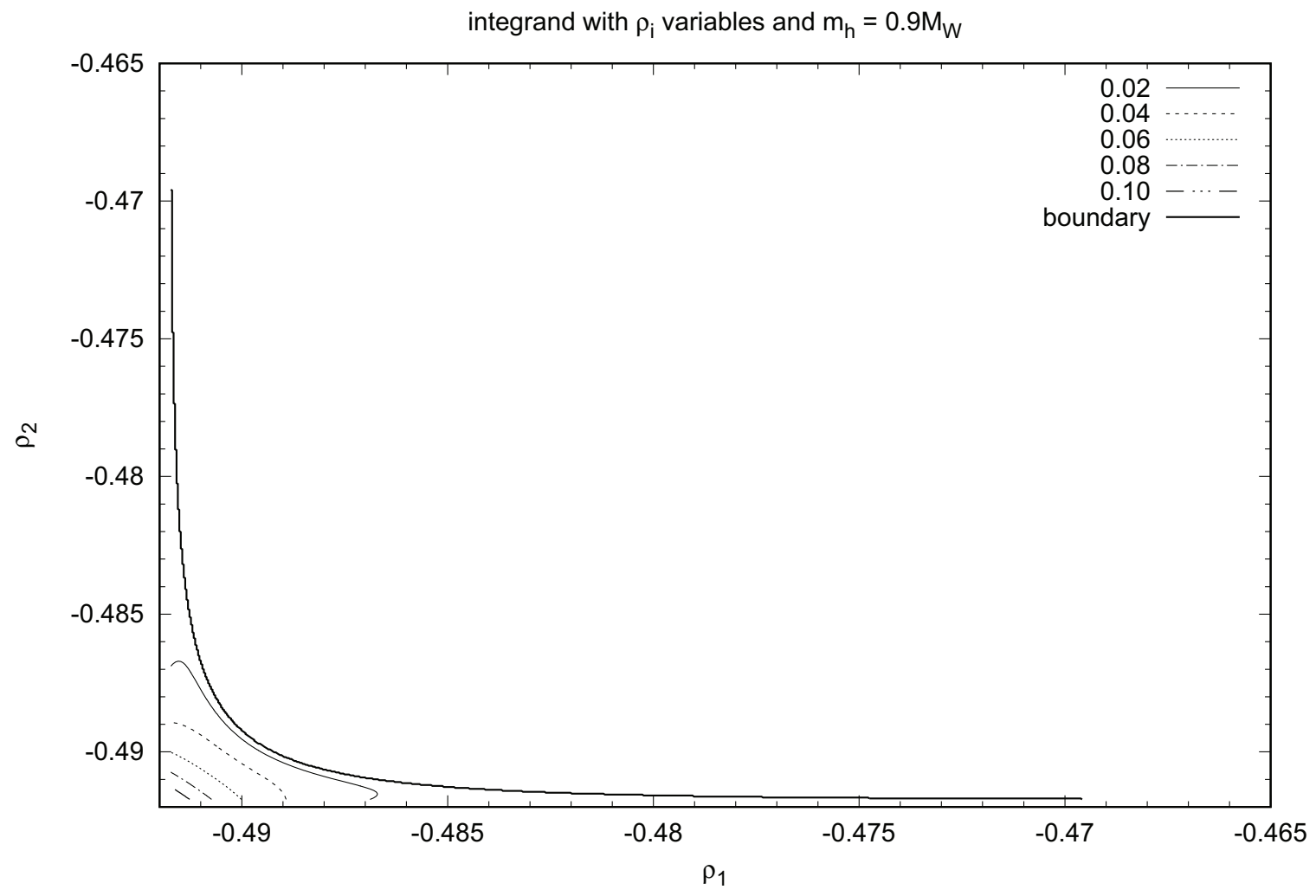

Figure 41: Contours of the integrand with respect to $\rho_{i}$ for $H_{i} \rightarrow W^{+} W^{-}$with $m_{H_{i}}=0.9 M_{W} \cdot \rho_{1}$ and $\rho_{2}$ are dimensionless, and the integrand has units of GeV. For $m_{H_{i}}<M_{W}$, the integrand is fairly well-behaved. As $m_{H_{i}} \rightarrow M_{W}$, however, the region of integration starts to develop "tails", which extend upward and to the side. 


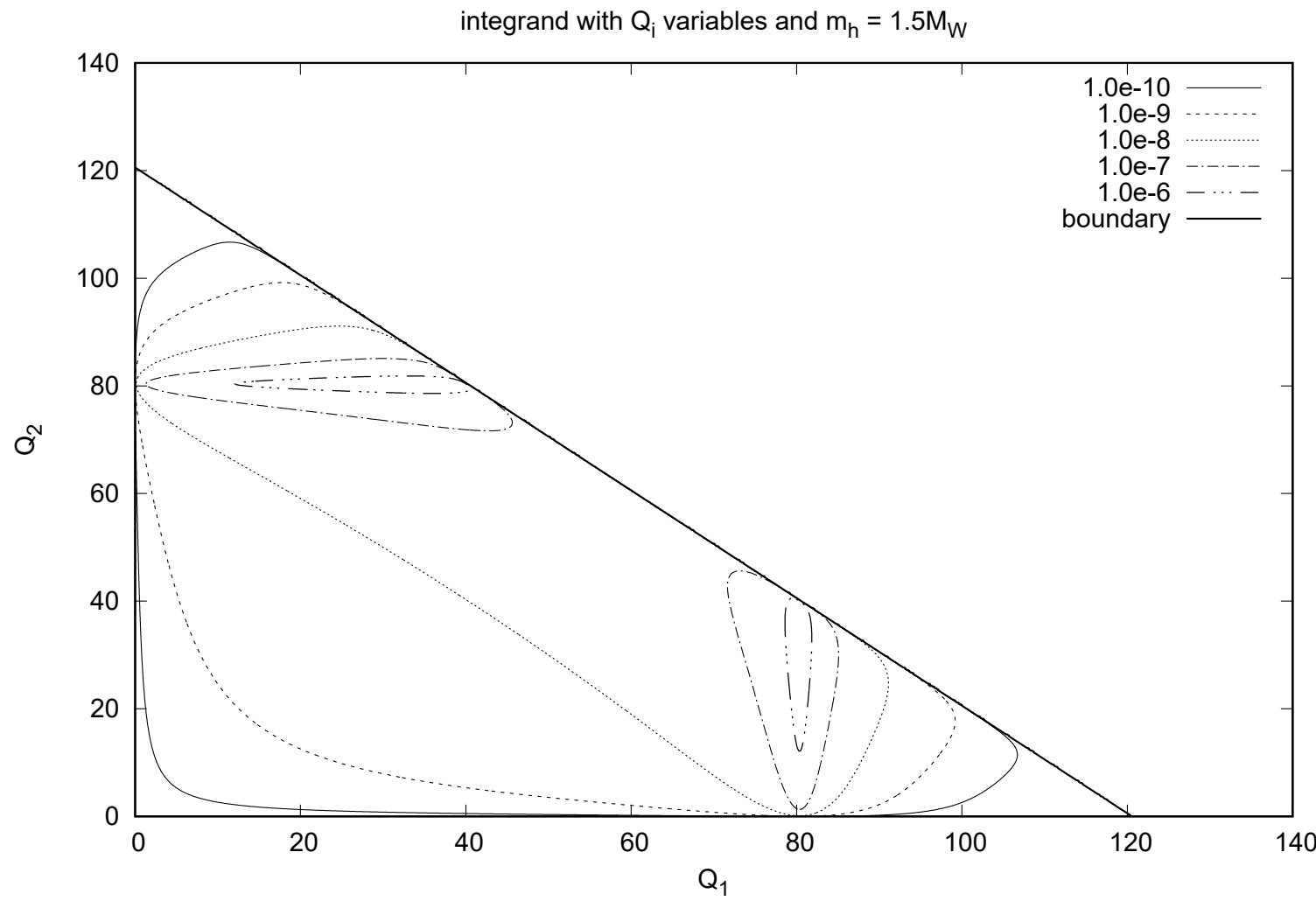

Figure 42: Contours of the integrand with respect to $Q_{i}$ for $H_{i} \rightarrow W^{+} W^{-}$with $m_{H_{i}}=1.5 M_{W} \cdot Q_{1}$ and $Q_{2}$ have units of $\mathrm{GeV}$, and the integrand has units of $[\mathrm{GeV}]^{-1}$. The integrand peaks where $Q_{1}=M_{W}$ or $Q_{2}=M_{W}$. However, so long as $m_{H_{i}}<2 M_{W}$, the integral is still manageable. 


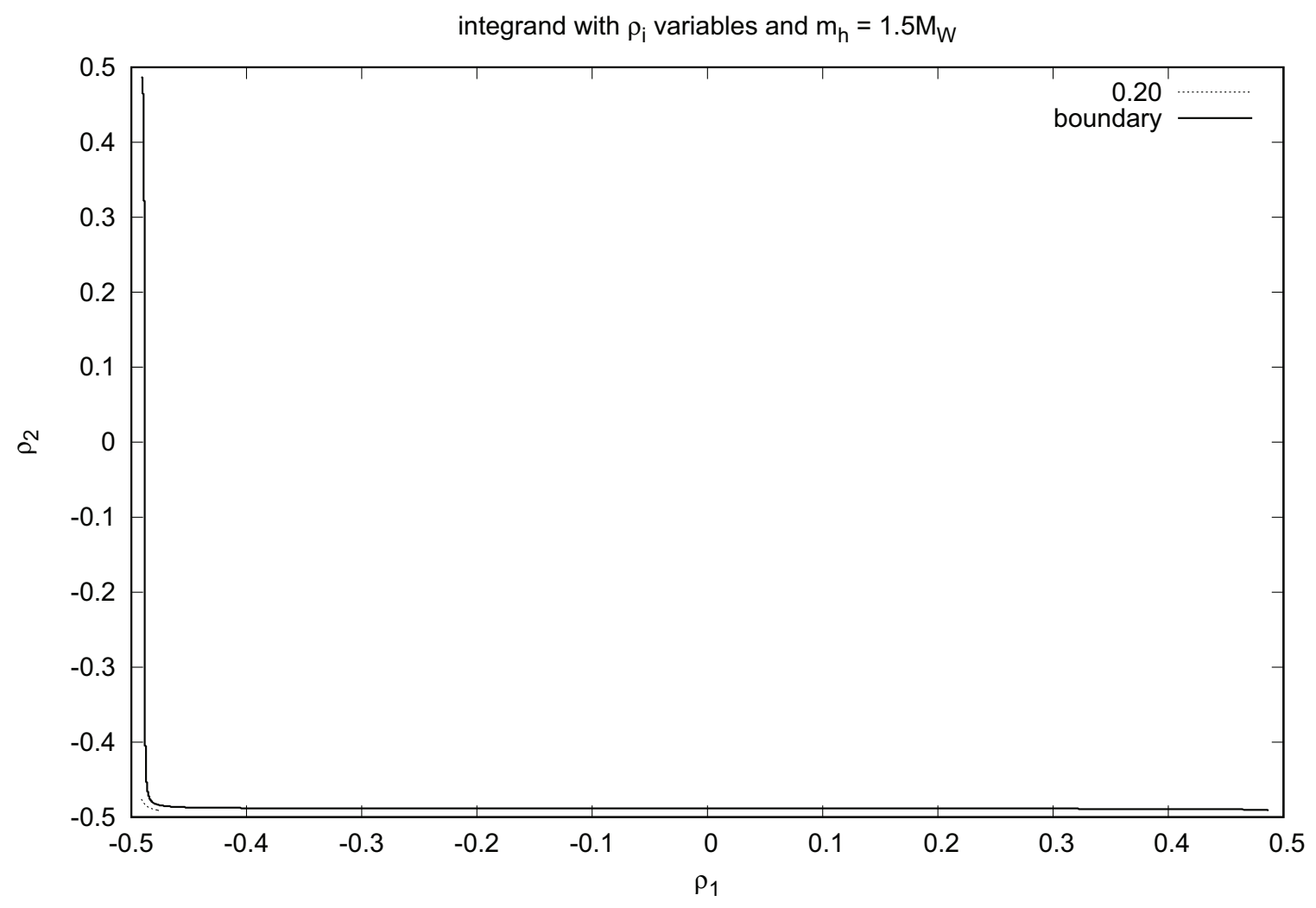

Figure 43: Contours of the integrand with respect to $\rho_{i}$ for $H_{i} \rightarrow W^{+} W^{-}$with $m_{H_{i}}=1.5 M_{W} \cdot \rho_{1}$ and $\rho_{2}$ are dimensionless, and the integrand has units of GeV. For $M_{W}<m_{H_{i}}<2 M_{W}$, the region of integration possesses "tails", which extend upward from $(-0.5,-0.5)$ to $(-0.5,+0.5)$, and to the side from $(-0.5,-0.5)$ to $(+0.5,-0.5)$. The integrand is largest near $(-0.5,-0.5)$, reaching values of 0.495 , while in the extended tails the integrand is on the order of 0.1 . 


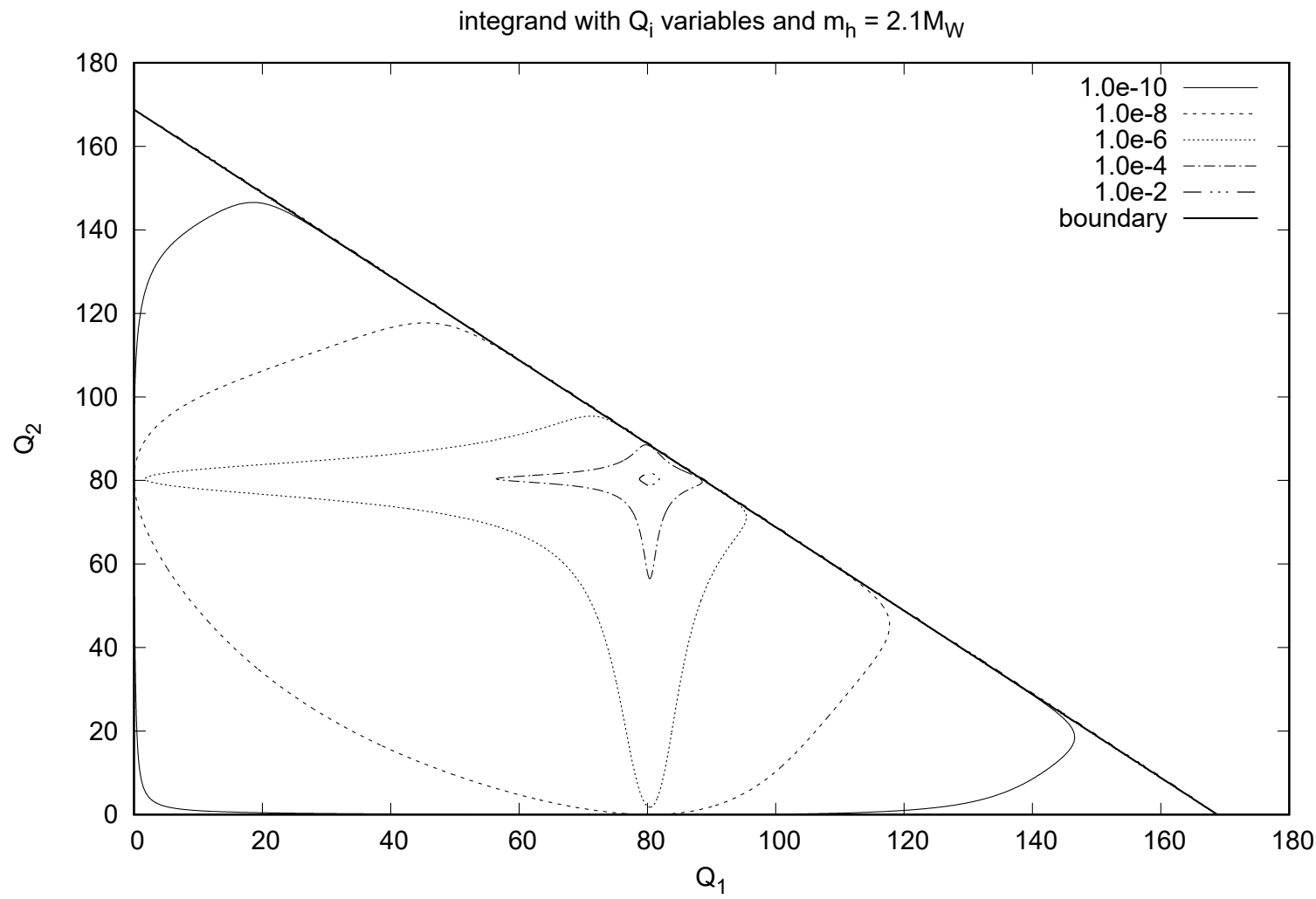

Figure 44: Contours of the integrand with respect to $Q_{i}$ for $H_{i} \rightarrow W^{+} W^{-}$with $m_{H_{i}}=2.1 M_{W} \cdot Q_{1}$ and $Q_{2}$ have units of $\mathrm{GeV}$, and the integrand has units of $[\mathrm{GeV}]^{-1}$. Once $m_{H_{i}}>2 M_{W}$, the integrand becomes severely peaked at $\left(Q_{1}, Q_{2}\right)=\left(M_{W}, M_{W}\right)$; the majority of the value of the integral comes from the area around this point. 


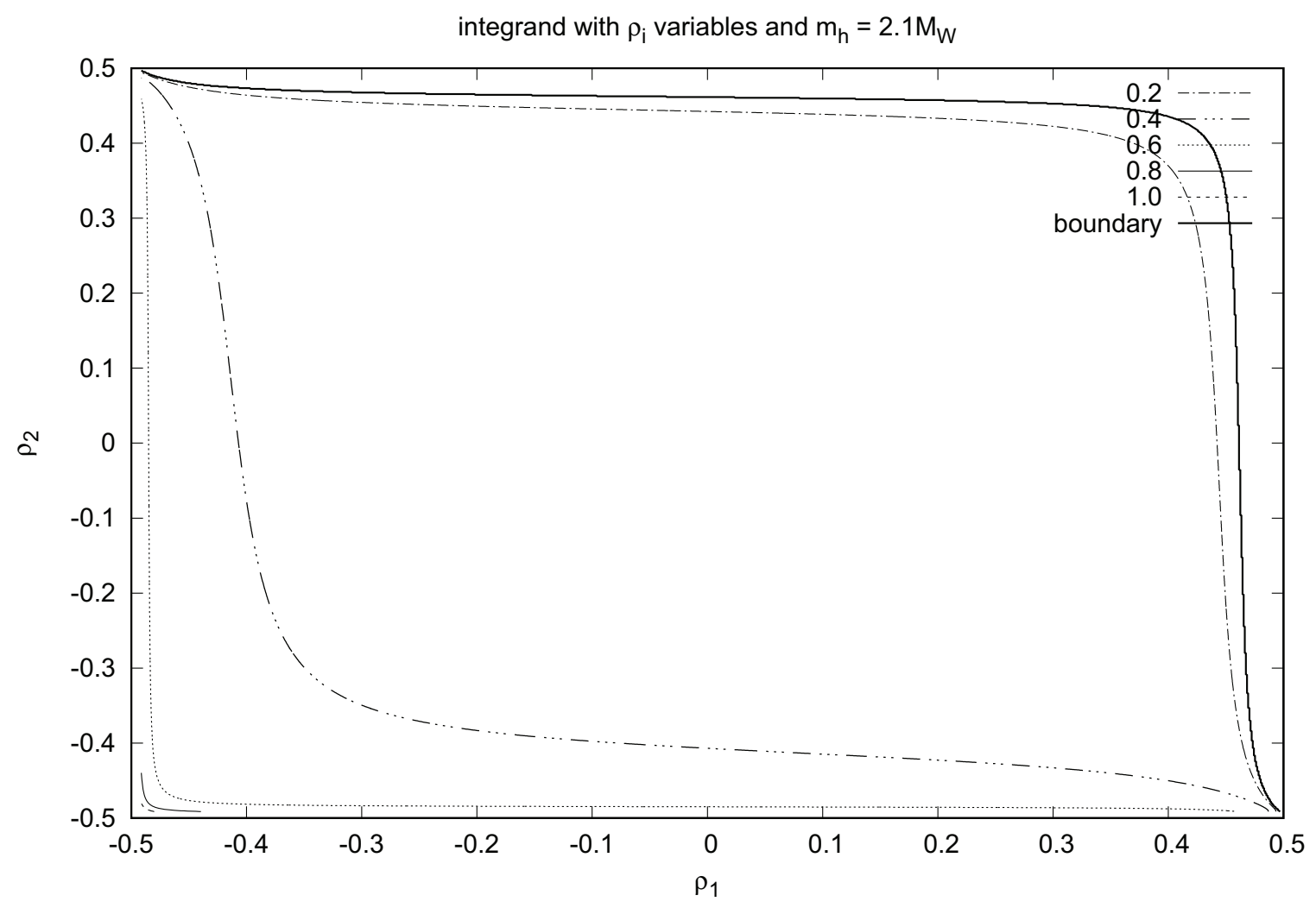

Figure 45: Contours of the integrand with respect to $\rho_{i}$ for $H_{i} \rightarrow W^{+} W^{-}$with $m_{H_{i}}=2.1 M_{W} \cdot \rho_{1}$ and $\rho_{2}$ are dimensionless, and the integrand has units of GeV. For $m_{H_{i}}>2 M_{W}$, the region of integration acquires a roughly rectangular shape, and the integrand is fairly uniform over the majority of this region, making $\rho_{i}$ a good choice of variables for numerical integration. 
$H_{i} \rightarrow V_{1} V_{2}$, we used Higgs decay partial width values computed by HDECAY 6.42 as the standard for comparison [22]. As a program for calculating extended Higgs particle behaviour, HDECAY has been around for more than two decades, so its detail and level of accuracy are well known and it is widely considered to be a gold standard for these sorts of accuracy tests. Using the data from HDECAY, we were then able to determine the relative accuracy of the original rectangular integration algorithm, as well as the accuracy of the new routines we were testing.

\subsection{Gauss-Legendre quadrature}

We began by checking out Gauss-Legendre quadrature. This method works as follows: First, it rescales the variables of integration so that they range from -1 to 1 in each variable. Then, it takes a fixed set of points $x_{1}, x_{2}, x_{3}, \ldots$ within that range and finds the value of the integrand $f(x)$ at those points. Finally, it applies a fixed weight $w_{1}, w_{2}, w_{3}, \ldots$ to each of these values in order to get the final integral:

$$
I=\sum_{i=1}^{n} w_{i} f\left(x_{i}\right)
$$

In $n$-point Gauss-Legendre quadrature (which takes $n$ different values for each variable to be integrated over) the values of $x_{i}$ and $w_{i}$ are chosen such that, if the integrand is a polynomial of degree $\leq 2 n-1$ with respect to a given variable, then integrating with respect to that variable will yield the exact result (for a more detailed explanation of Gauss-Legendre quadrature, see Appendix A). As such, if an integrand is sufficiently well-behaved that it can be approximated by a degree $2 n-1$ polynomial, then GaussLegendre quadrature can compute the value of its integral to a high degree of precision.

For $m_{H_{i}}>M_{V_{1}}+M_{V_{2}}$, 16-point Gauss-Legendre quadrature applied to the integrand in terms of $\rho_{i}$ yields an accuracy at least as good as the original integration routine (see Figs. 46 and 47). Since the original routine sampled $100 \times 100$ points, 


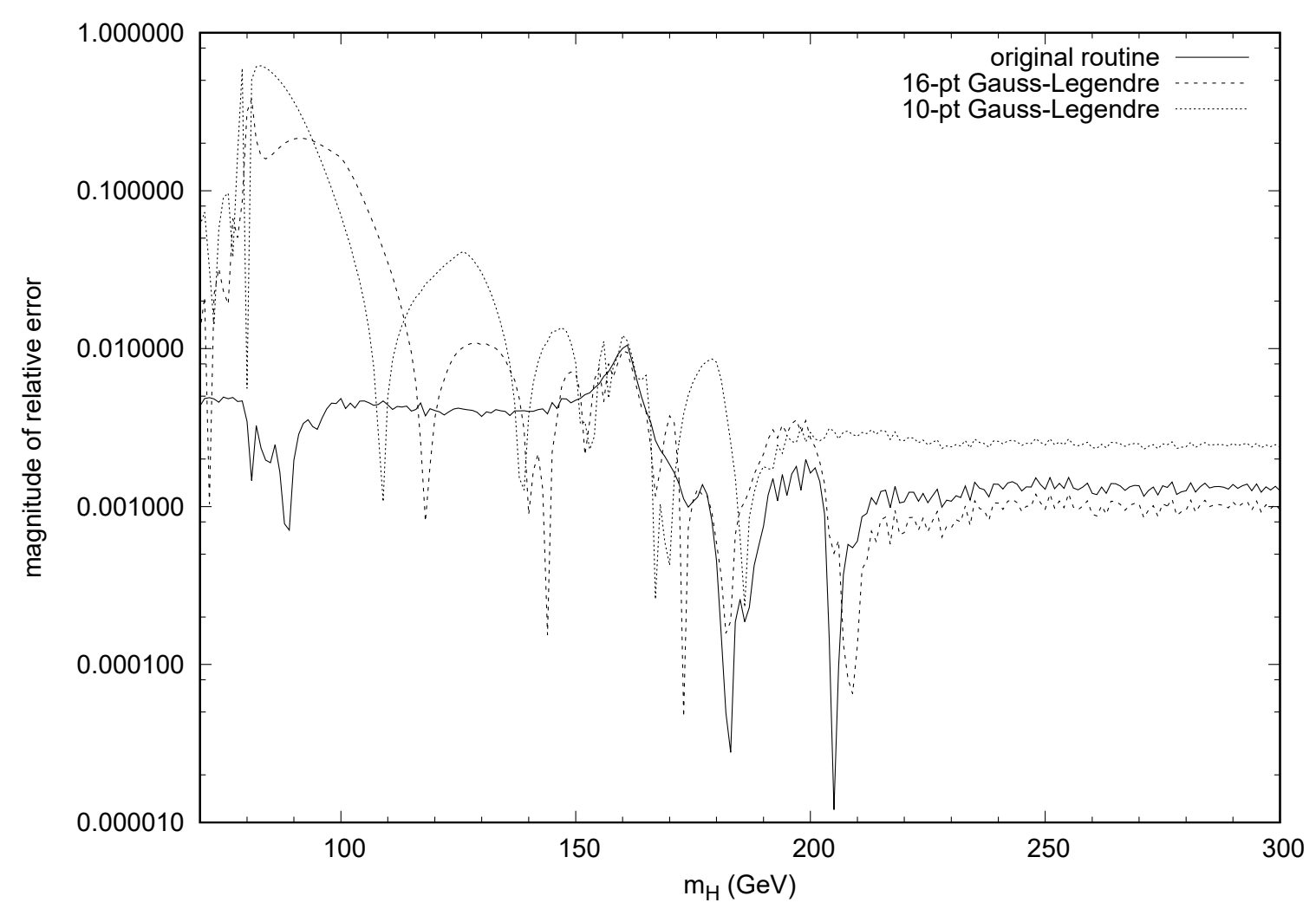

Figure 46: Magnitude of the relative error of $H_{i} \rightarrow W^{+} W^{-}$using the original integration routine, as well as 10-point and 16-point Gauss-Legendre quadrature, as compared to the values computed by HDECAY 6.42.

and the 16-point Gauss-Legendre routine uses $16 \times 16$ points, this leads to a nearly forty-fold speedup in efficiency. However, for the reasons stated above, the GaussLegendre method fails for $m_{H_{i}}<M_{V_{1}}+M_{V_{2}}$. Hypothetically, one might be able to divide the integration region into smaller pieces and handle them individually, but the behaviour of the integration region tails (e.g. tail thickness, speed at which the integrand drops off as one approaches the ends of the tails) tends to vary significantly between $0 \mathrm{GeV}<m_{H_{i}}<2 M_{V}$; as such, an integration routine which accounts for all this variation would need to be unnecessarily complicated, involving a lot of magic number fine-tuning in order to be properly optimized. Integrating with respect to $Q_{i}$ doesn't solve the problem either: because the integrand increases by a couple orders of magnitude in the narrow regions around $Q_{i}=M_{V_{i}}$, Gauss-Legendre quadrature cannot accurately calculate the integral without using a number of points comparable 


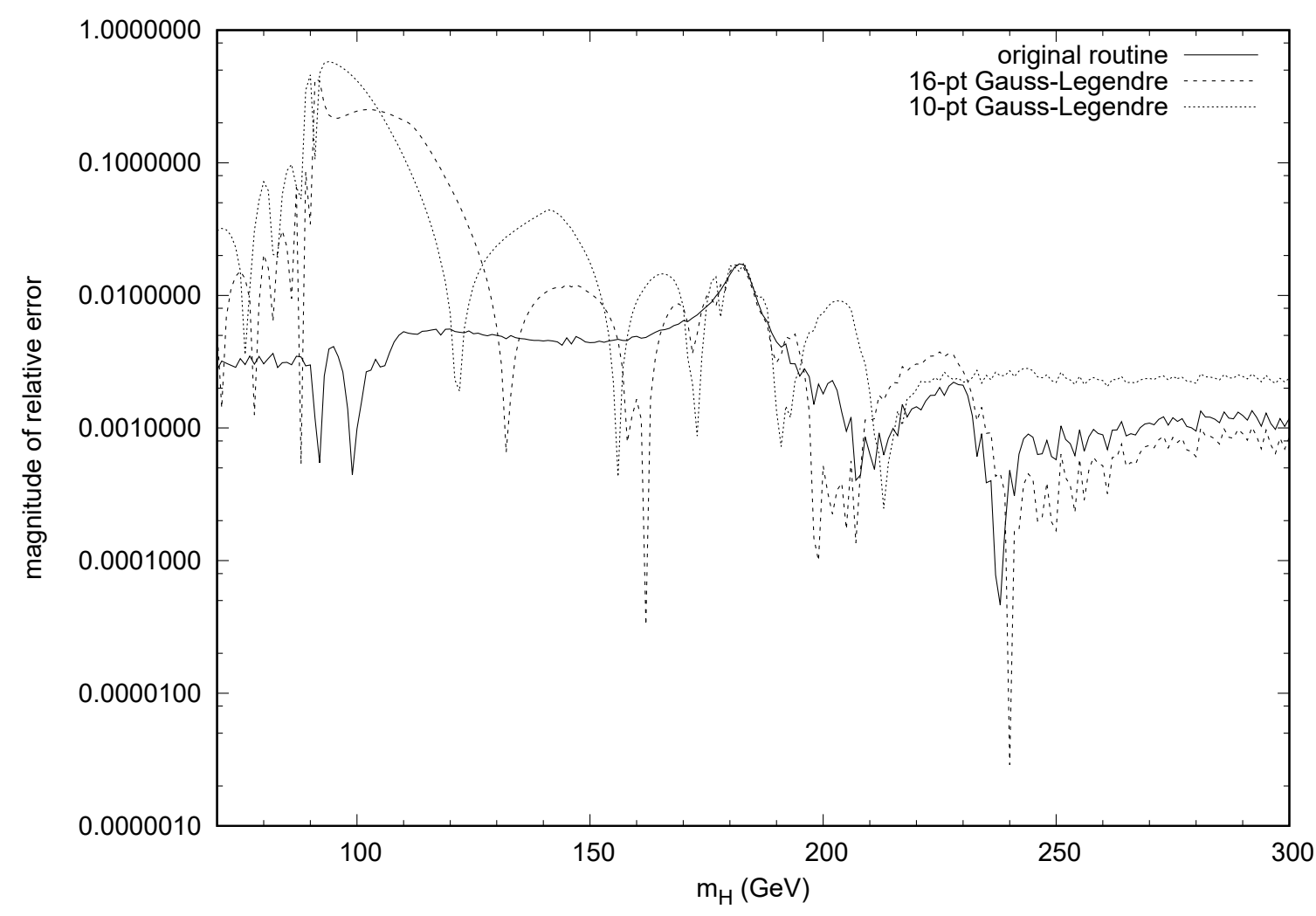

Figure 47: Magnitude of the relative error of $H_{i} \rightarrow Z Z$ using the original integration routine, as well as 10-point and 16-point Gauss-Legendre quadrature, as compared to the values computed by HDECAY 6.42. 
to that used by the original routine. To properly compute the integral for $H_{i} \rightarrow V_{1} V_{2}$ in this mass region, we turn to a new routine: the VEGAS algorithm.

\subsection{The VEGAS routine}

For our work, we used an implementation of the VEGAS algorithm [23] which we borrowed with permission from PROSPINO [24]. The VEGAS algorithm operates by sampling random points in an $n$-dimensional hyperrectangle integration region (if the integration region is not rectangular, then one must create a hyperrectangle that contains the integration region, and assume that the integrand is zero for all points which do not fall within the integration region). The integration region is divided into a grid of hyperrectangles, and a random sampling of points is used to determine where the integrand is largest; this information about the integrand is then used to weight the sampling distribution on subsequent passes. The integrand is assumed to have the form

$$
f\left(x_{1}, x_{2}, \ldots, x_{n}\right)=f_{1}\left(x_{1}\right) f_{2}\left(x_{2}\right) \ldots f_{n}\left(x_{n}\right)
$$

to simplify the construction of the sampling distribution. The actual integrand cannot differ too much from this assumption, in order for the VEGAS routine to work. Once the sampling distribution has been determined, several more random samplings are taken, but this time with the intent to estimate the actual value of the integral. Once the integral has been calculated to the desired level of accuracy (or the maximum allowed number of random samplings has been taken), then the final estimate is output by the algorithm.

Because of the shape of the integrand when we integrate with respect to $\rho_{i}$ for $m_{H}<M_{V_{1}}+M_{V_{2}}$ (see Fig. 43), attempting to contain the integration region within a rectangle results in a region that is mostly empty space. As a result, there is a strong probability that the integration region will be missed by random samplings of the 
VEGAS routine, unless a prohibitive number of points are used. However, when we integrate with respect to $Q_{i}$, the integration region is triangular and always fills half of the rectangular region we will sample from when implementing the VEGAS routine. Since the Breit-Wigner distributions in Eq. (81) make the biggest contributions to the magnitude of the integrand, the assumption in (87) is approximately fulfilled, and the VEGAS routine should run well.

There are two main factors that determine the accuracy and computing time of the VEGAS routine: number of points taken per sampling and maximum number of sampling iterations. We do need a reasonable benchmark to compare the VEGAS routine to, though, and in this case, when the VEGAS routine is chosen specifically for accuracy (i.e. we do a run with 10,000, 000 sample points and 30 iterations) we find that its results are in fact closer to the original routine than they are to HDECAY, as seen in Fig. 48. Since the VEGAS routine values seem to converge more easily to the values produced by the original routine, we will use the original as our point of comparison, rather than HDECAY.

The maximum relative error between values produced by the VEGAS routine and the original routine is catalogued in Fig. 49 for a broad range of possible numbers of iterations and numbers of sampling points. From the figure we can see that, while the error initially decreases as the iterations and sampling points increase, it levels off to around 0.009 when the number of sampling points is greater than 6000 and the number of iterations is larger than 3. However, we notice from Fig. 48 that there is a similarly large difference between VEGAS and the original routine in the region $80 \mathrm{GeV}<$ $m_{H_{i}}<100 \mathrm{GeV}$, even though the VEGAS routine is using $10^{7}$ sampling points and thirty iterations, while the original routine uses only $1,000 \times 1,000=10^{6}$ points. As such, it may be more productive to run the VEGAS routine once with a large number of points and iterations, and compute the accuracy of our other runs relative to that. Using $10^{6}$ points and 30 iterations for our standard of comparison (since it was faster 


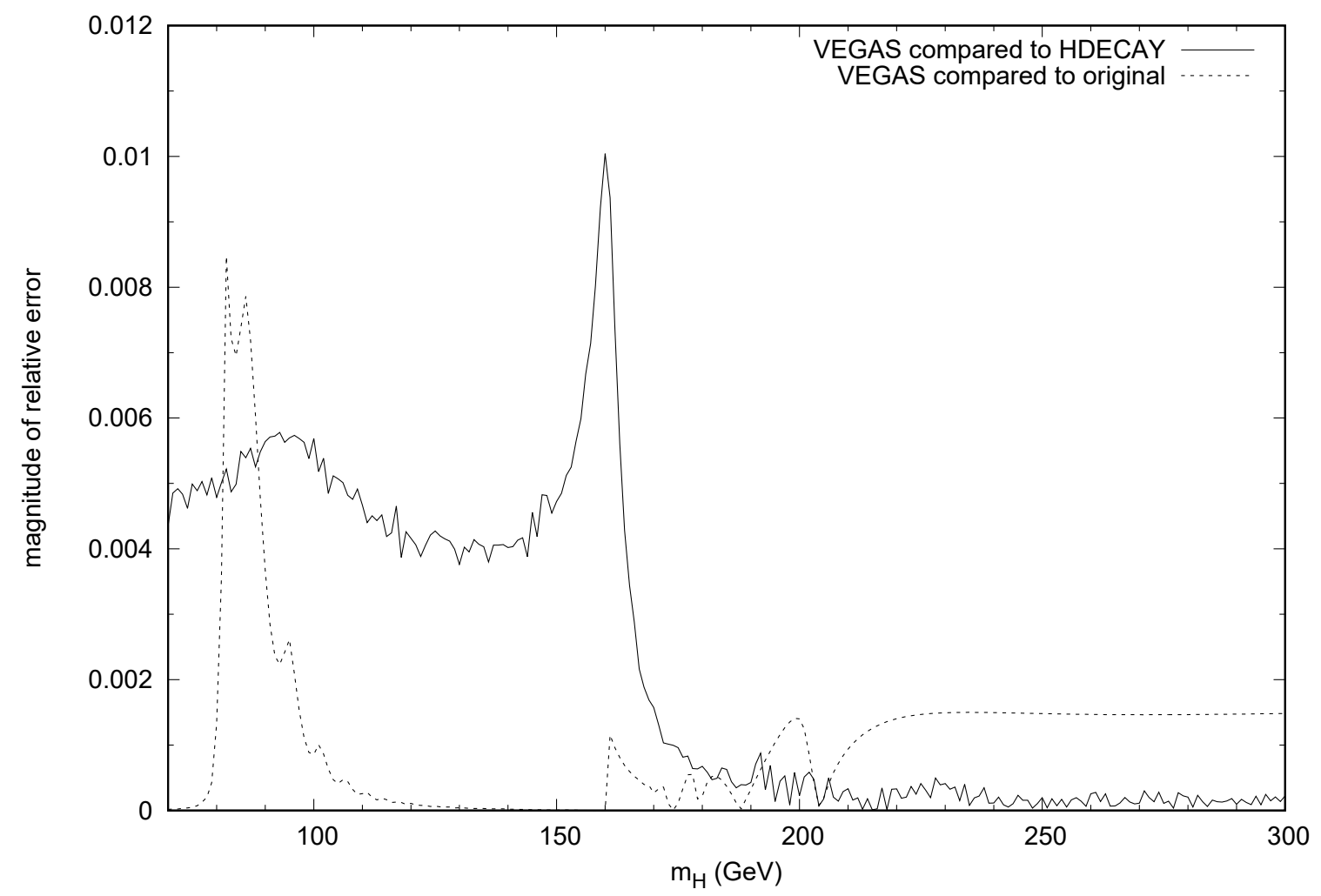

Figure 48: Magnitude of the relative error of $\Gamma\left(H_{i} \rightarrow W^{+} W^{-}\right)$using the VEGAS routine, as compared to the values computed by HDECAY 6.42 and the original integration routine. 


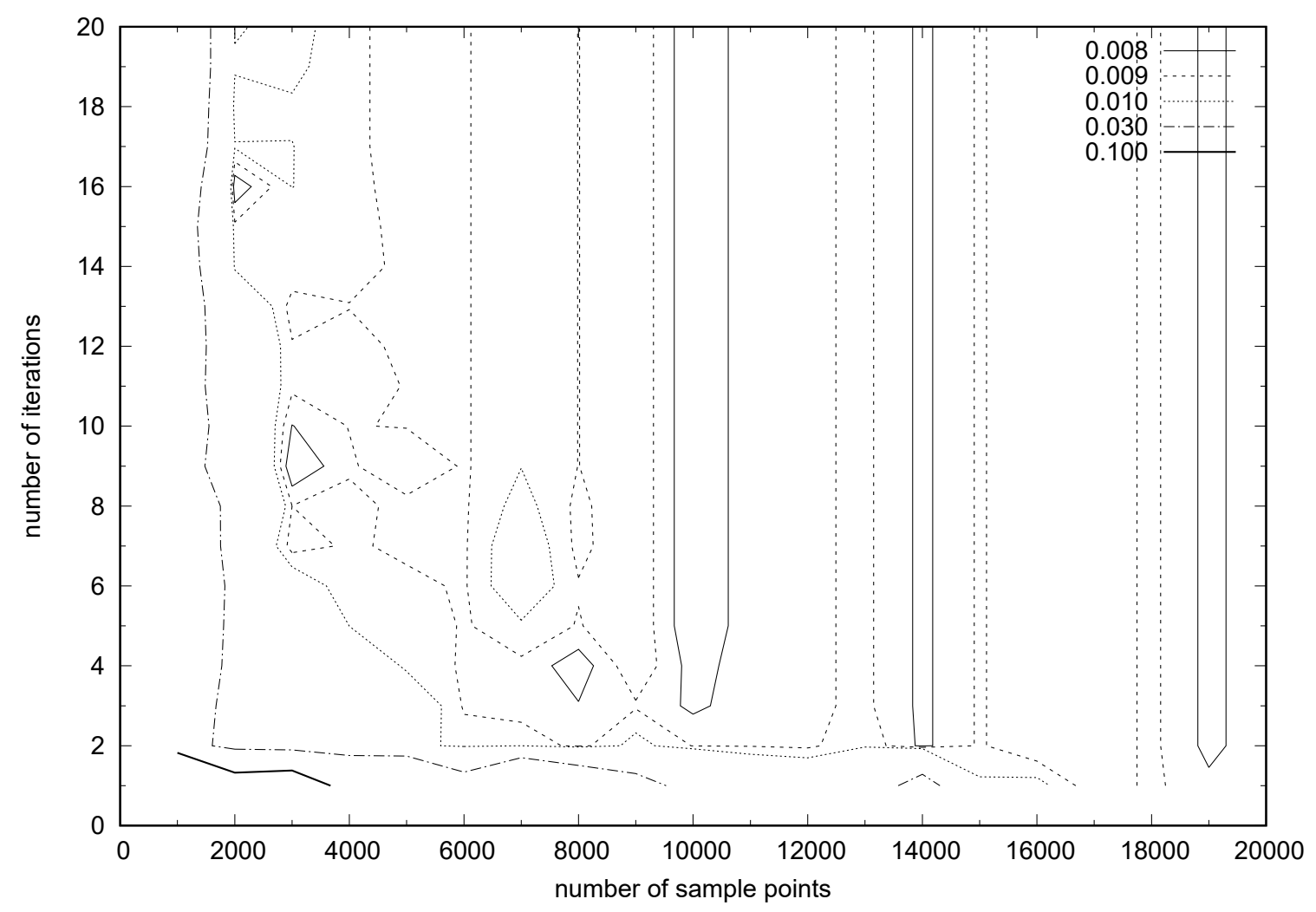

Figure 49: For each choice of number of sampling points and number of iterations, the VEGAS routine is used to compute $\Gamma\left(H_{i} \rightarrow W^{+} W^{-}\right)$and $\Gamma\left(H_{i} \rightarrow Z Z\right)$ for $m_{H_{i}}$ ranging from $70 \mathrm{GeV}$ to $300 \mathrm{GeV}$, taken in $1 \mathrm{GeV}$ increments. These values are compared to the same values computed from the original routine, and the largest proportionate difference is added to the figure at the appropriate point. This maximum difference is largest for small numbers of sampling points and iterations, but becomes relatively flat when the number of sampling points is greater than 6000 and the number of iterations is larger than 3 . 


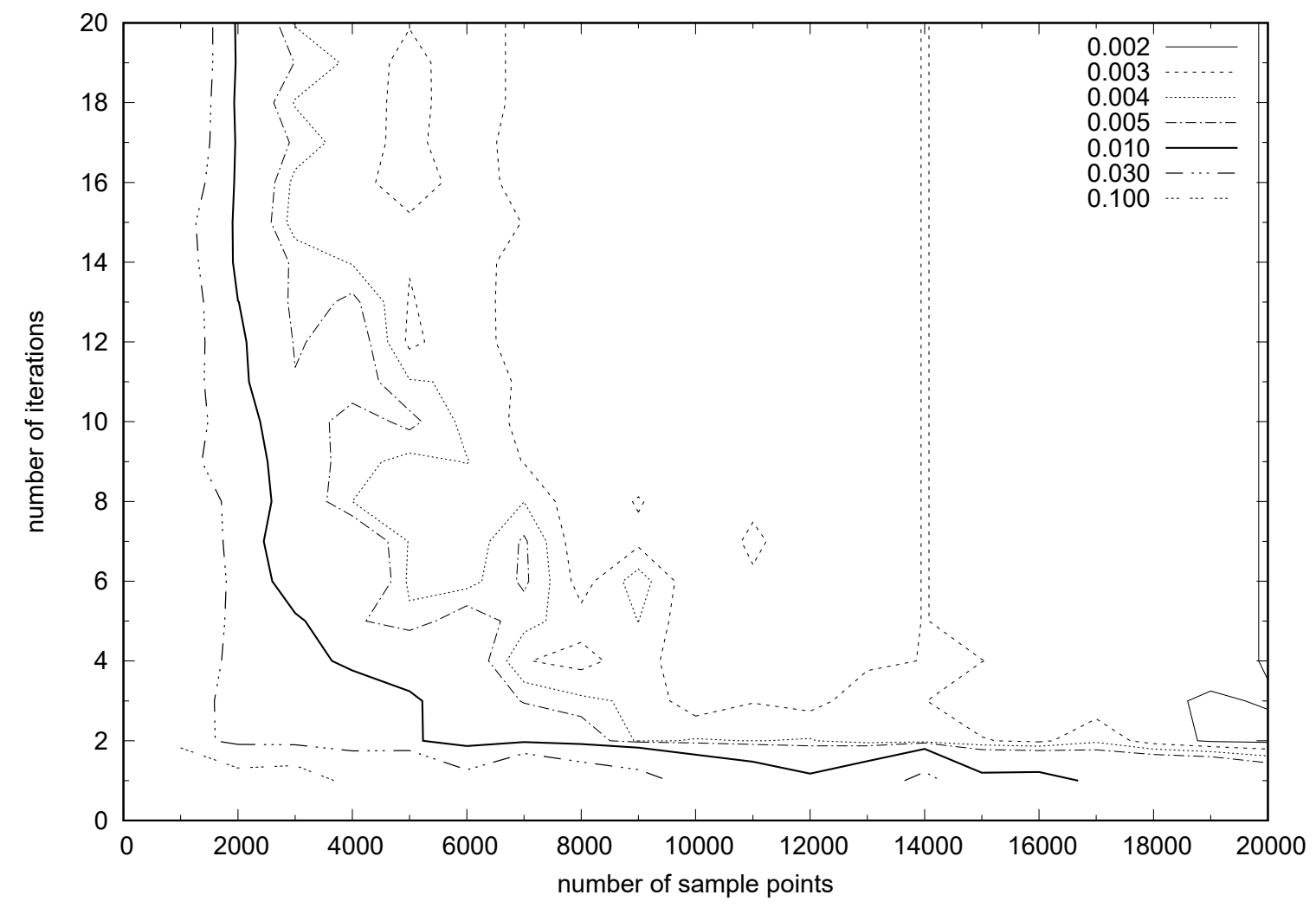

Figure 50: For each choice of number of sampling points and number of iterations, the VEGAS routine is used to compute $\Gamma\left(H_{i} \rightarrow W^{+} W^{-}\right)$and $\Gamma\left(H_{i} \rightarrow Z Z\right)$ for $m_{H_{i}}$ ranging from $70 \mathrm{GeV}$ to $300 \mathrm{GeV}$, taken in $1 \mathrm{GeV}$ increments. These values are compared to the same values computed for a run of the VEGAS routine with $10^{6}$ points and 30 iterations, and the largest proportionate difference is added to the figure at the appropriate point. This maximum difference is largest for small numbers of sampling points and iterations, but becomes relatively flat when the number of sampling points is greater than 10,000 and the number of iterations is larger than 3 .

and the drop in accuracy compared to $10^{7}$ points is inconsequential), we have checked the accuracy of VEGAS and show the results in Fig. 50. From this figure, we can see that, in order to ensure that we are not missing out on any potentially increased accuracy, we would do best to use 10,000 sampling points and 3 sampling iterations when we run VEGAS.

Finally, we will measure how long the VEGAS routine takes to run (see Fig. 51), performing the computations on a single core of an Intel Celeron CPU N2840, at a base frequency of $2.16 \mathrm{GHz}$. For 1,000 to 20,000 sample points, and 1 to 20 iterations of sampling, the time required to compute $\Gamma\left(H_{i} \rightarrow W^{+} W^{-}\right)$and $\Gamma\left(H_{i} \rightarrow Z Z\right)$ for 


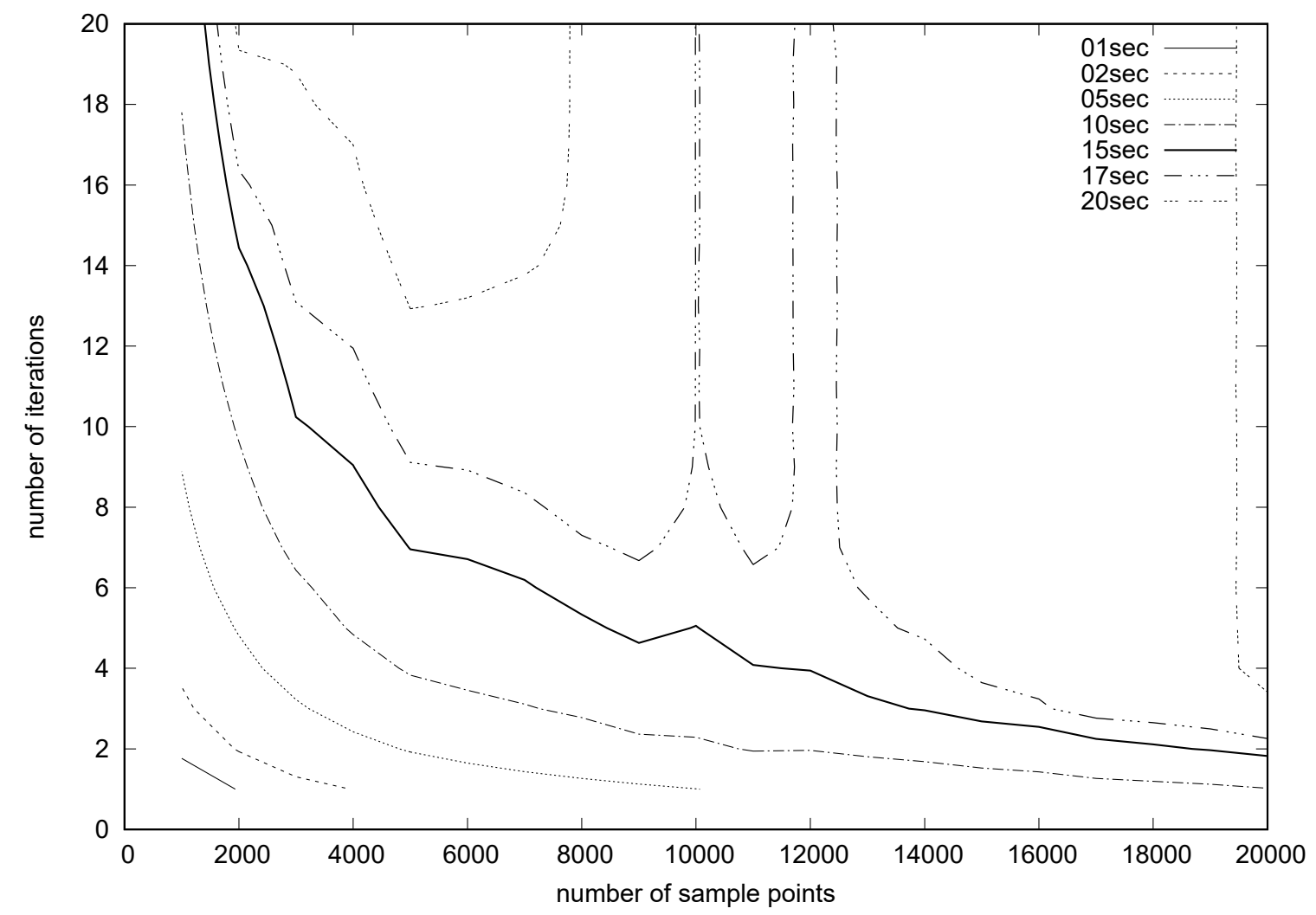

Figure 51: Time taken to run the VEGAS routine for values of $m_{H_{i}}$ from $70 \mathrm{GeV}$ to $300 \mathrm{GeV}$ in $1 \mathrm{GeV}$ increments, where the number of sample points and number of iterations is varied. For comparison, the original routine takes 152 seconds to perform the same task.

values of $m_{H_{i}}$ between $70 \mathrm{GeV}$ and $300 \mathrm{GeV}$, in increments of $1 \mathrm{GeV}$, never takes more than 21 seconds. When the number of sampling points is 10,000 and the number of iterations is 3 , the time taken goes down to 12 seconds. For comparison, accomplishing the same task with the original integration routine requires 152 seconds. As such, the VEGAS algorithm gives us a more than twelve-fold speedup relative to the original.

\subsection{Final integration routine}

Now that we have all the necessary data, we have combined the VEGAS routine and the Gauss-Legendre routine into a single entity. For $m_{H_{i}} \leq M_{V_{1}}+M_{V_{2}}$, we use the VEGAS routine with 10,000 sampling points and 3 sampling iterations, while for $m_{H_{i}}>M_{V_{1}}+M_{V_{2}}$, we use the 16-point Gauss-Legendre quadrature routine. The 


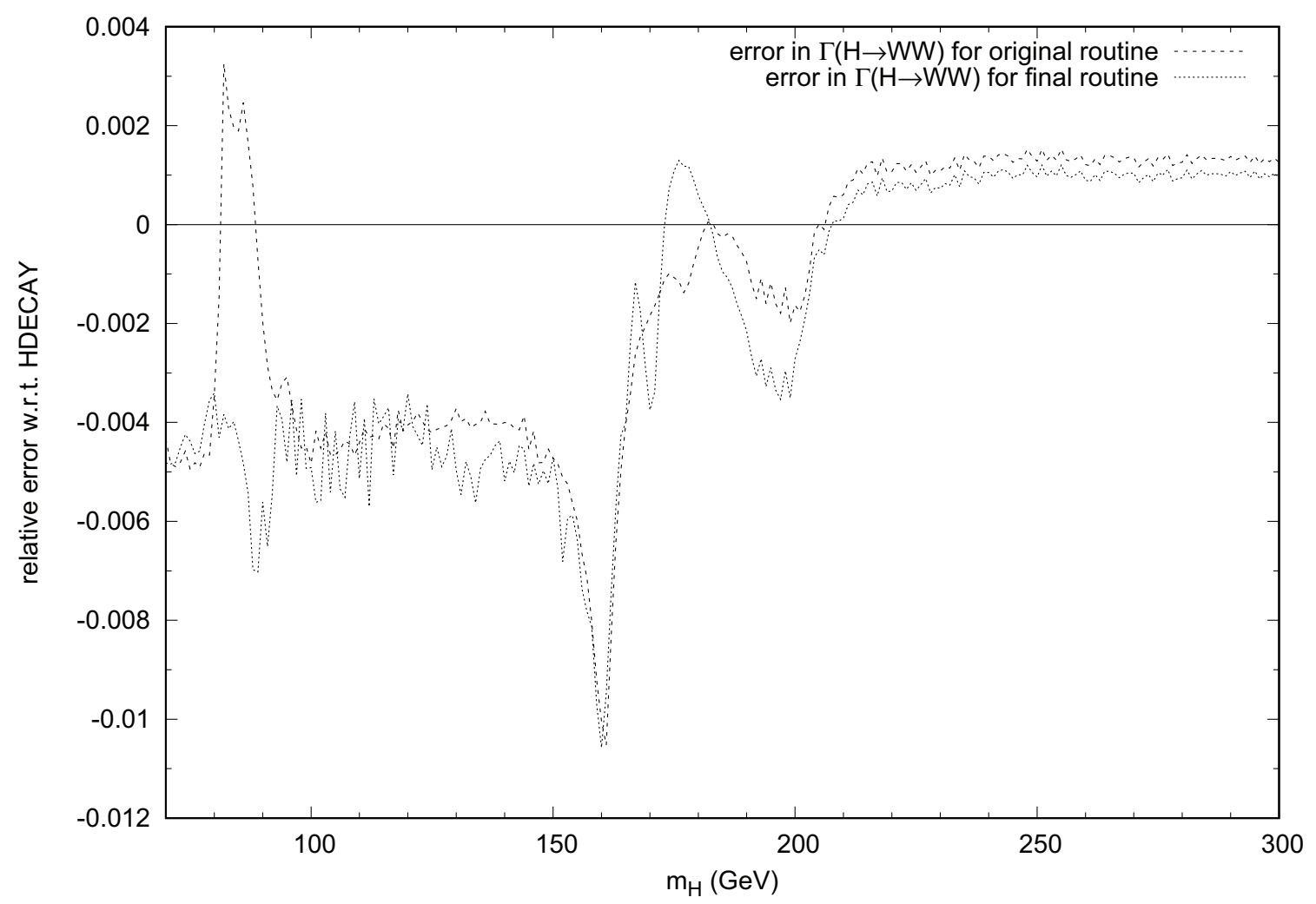

Figure 52: Relative error of $\Gamma\left(H_{i} \rightarrow W W\right)$ compared to HDECAY in original and final routine.

resulting accuracy relative to HDECAY, as well as runtime information, are shown in Figs. 52, 53, and 54. As can be seen from the figures, we have managed to maintain the same level of accuracy as the original routine, while achieving a speedup of twentyto fortyfold. It is our hope that future work will enable us to implement similar routines for some of the other decays, such as $H_{3}^{0} \rightarrow t \bar{t}$ and $H_{3}^{+} \rightarrow t \bar{b}$. 


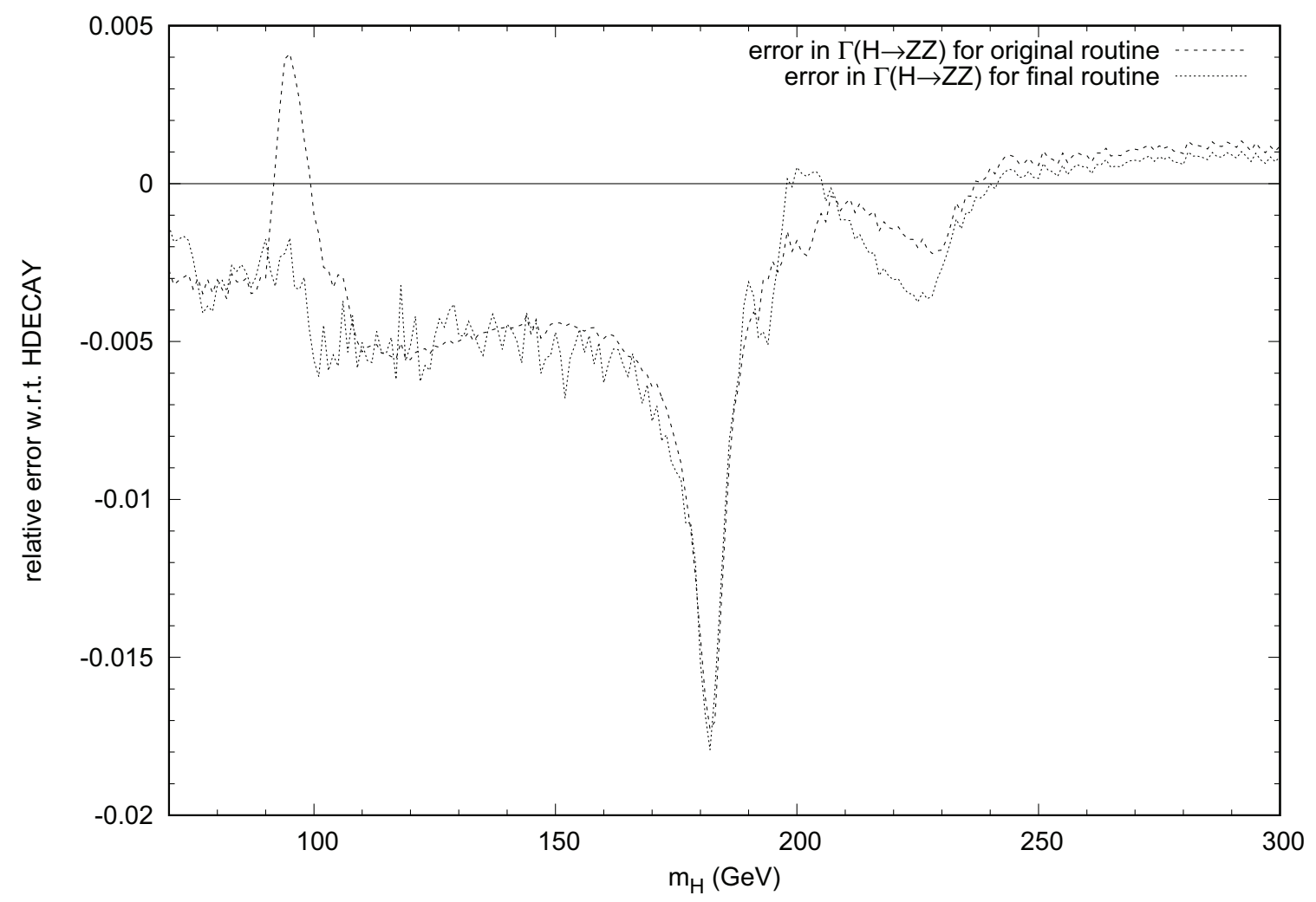

Figure 53: Relative error of $\Gamma\left(H_{i} \rightarrow Z Z\right)$ compared to HDECAY in original and final routine. 


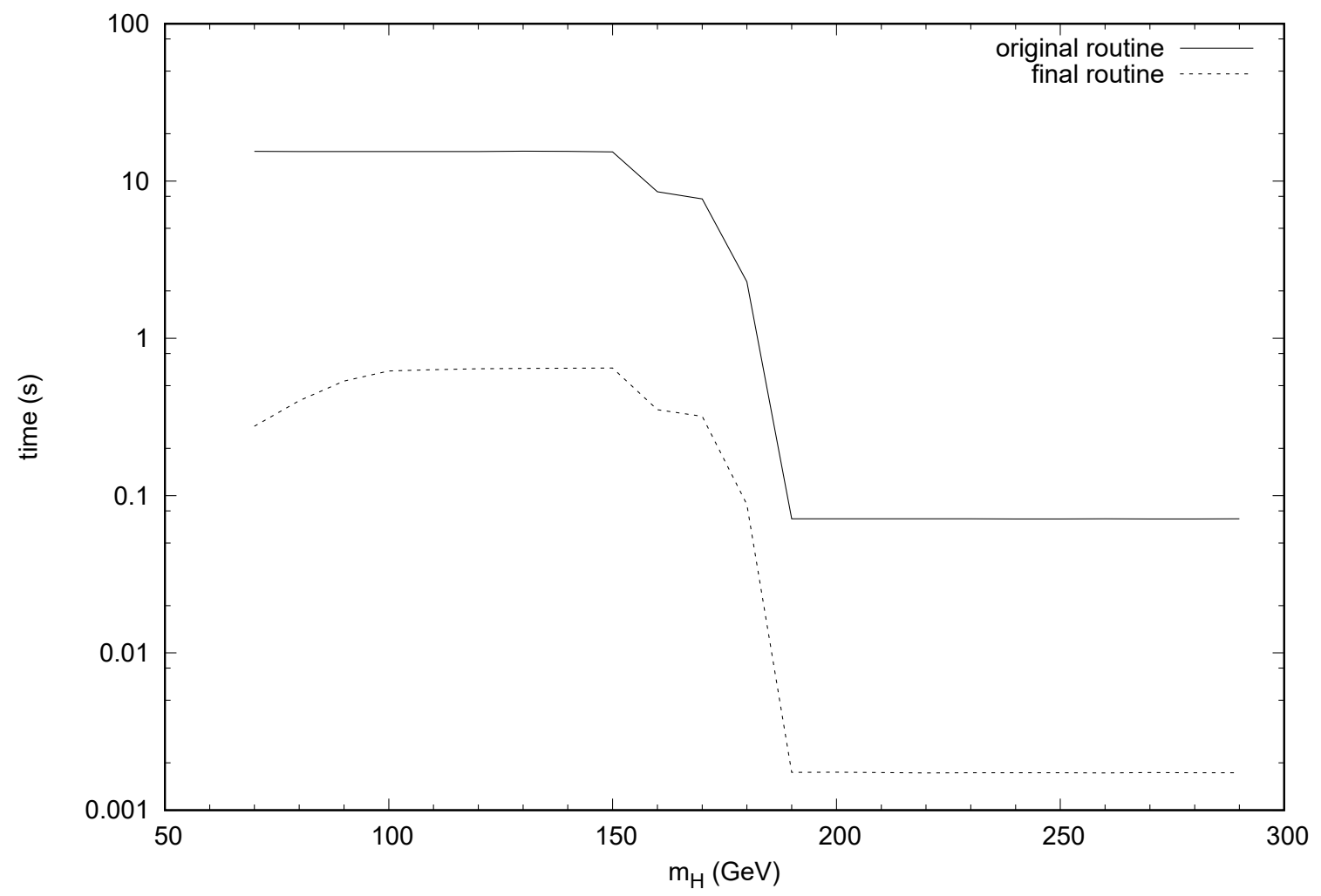

Figure 54: The original and final routines are timed between $m_{H_{i}}=70 \mathrm{GeV}$ and $m_{H_{i}}=300 \mathrm{GeV}$ to determine how long it takes them to compute $\Gamma\left(H_{i} \rightarrow W W\right)$ and $\Gamma\left(H_{i} \rightarrow Z Z\right)$ ten times. For $m_{H_{i}}<M_{V_{1}}+M_{V_{2}}$, original routine takes about 15 seconds, while the final routine takes 0.64 seconds. For $m_{H_{i}}>M_{V_{1}}+M_{V_{2}}$, the original routine takes 0.071 seconds and the final routine takes 0.0017 seconds. 


\section{Conclusions}

In the course of this thesis we have studied the constraints on and phenomenological behaviour of the H5plane benchmark scenario in the GM model. The H5plane benchmark has two free parameters: $m_{5}$, the mass of the $H_{5}$ Higgs particles; and $s_{H}$, where $s_{H}^{2}$ is equal to the fraction of $M_{W}^{2}$ and $M_{Z}^{2}$ that is generated by the VEV of the isospin triplet scalar fields. The H5plane benchmark is defined for $m_{5} \in[200,3000] \mathrm{GeV}$. Existing theoretical and experimental constraints limit $s_{H}$ to be below 0.55 in the H5plane benchmark, so that at most $30 \%$ of the $W$ and $Z$ boson squared-masses can be generated by the triplets. A full parameter scan yields an allowed region in the $m_{5^{-}}$ $s_{H}$ plane only slightly larger than in the H5plane benchmark for $m_{5} \in[200,3000] \mathrm{GeV}$, demonstrating that the majority of experimental behaviour predicted in a full scan of the GM model should not differ significantly from that displayed by the H5plane benchmark. However, one should be aware that some experimental predictions from a full scan can deviate significantly from experimental predictions for the H5plane benchmark; most notably, a full scan shows that $H_{3}-H_{5}$ and $H-H_{5}$ mass splittings show substantial variation in a full scan, while the same quantities show almost no variation at fixed $m_{5}$ in the H5plane benchmark. This result means that certain decay modes, i.e. those which involve one Higgs decaying into another, will likely show significant variation in their predicted rates over a full scan as compared to the H5plane predictions. However, most full scan predictions should still hew closely to those of the H5plane benchmark. The public code GMCALC 1.2.1, which computes the properties and behaviour of the particles in the GM model on the basis of a set of input parameters, was used to do our numerical work.

We showed that the couplings of the $125 \mathrm{GeV}$ Higgs boson $h$ in the H5plane benchmark are sufficiently SM-like that the benchmark is not further constrained by the ATLAS and CMS measurements of Higgs production and decay at LHC centerof-mass energies of 7 and $8 \mathrm{TeV}$ - in fact, over most of the H5plane benchmark, the 
fit to LHC data is slightly better than in the SM. Over the H5plane benchmark, the $h$ coupling to fermions can be suppressed (compared to it SM value) by up to $10 \%$ or enhanced by up to $1.4 \%$, its coupling to vector boson pairs can be enhanced by up to $21 \%$, and its loop-induced coupling to photon pairs can be suppressed by up to $1.3 \%$ or enhanced by up to $24 \%$ (loop diagrams involving the charged scalars in the GM model contribute non-negligibly to this). Due to a coincidental cancellation among the fermionic and bosonic contributions, the total width of $h$ can be suppressed by up to $2.9 \%$ or enhanced by up to $3.5 \%$ compared to that of the SM Higgs boson.

By design, the mass-degenerate $H_{5}^{ \pm \pm}, H_{5}^{ \pm}$, and $H_{5}^{0}$ scalars are the lightest new scalars in the H5plane benchmark, and hence decay only to vector boson pairs at tree level. Due to the parameter specifications in the benchmark, the mass splittings $m_{3}-m_{5}$ and $m_{H}-m_{5}$ are almost constant with $s_{H}$, depending primarily on $m_{5}$. They fall from maxima of $84 \mathrm{GeV}$ and $120 \mathrm{GeV}$, respectively, at $m_{5}=200 \mathrm{GeV}$ to minima of $7 \mathrm{GeV}$ and $9 \mathrm{GeV}$, respectively, at $m_{5}=3000 \mathrm{GeV}$. (These mass splittings vary much more freely in the full GM model.) While the mass-to-width ratios of all the new scalars in the GM model remain below $8 \%$ in the H5plane benchmark, the fairly small mass splitting between $H_{5}^{0}$ and $H$ means that these two resonances can overlap and interfere when they are produced in vector boson fusion and decay to $W^{+} W^{-}$or $Z Z$. Their mass splitting becomes smaller than their intrinsic widths when $m_{5} \gtrsim 700 \mathrm{GeV}$, unless $s_{H}$ is small.

We also studied the production and decays of the new heavy Higgs bosons in the GM model in the H5plane benchmark. We found that, due to coupling suppressions, the production cross section of $H$ in gluon fusion (vector boson fusion) can be at most $58 \%(4.8 \%)$ as large as that of a SM Higgs boson of the same mass. $H$ decays mainly to $W^{+} W^{-}$and $Z Z$ for $m_{5}$ below $600-1000 \mathrm{GeV}$ (depending on $s_{H}$ ), and mainly to $h h$ for $m_{5}$ above $700-1300 \mathrm{GeV}$. Its branching ratio to $t \bar{t}$ can top $30 \%$ for $m_{5}$ between 400 and $700 \mathrm{GeV}$. 
$H_{3}^{0}$ decays predominantly to $t \bar{t}$ from the kinematic threshold at $m_{5}=280 \mathrm{GeV}$ up to $m_{5} \simeq 500 \mathrm{GeV}$, where $h Z$ takes over as the dominant decay mode. Below the $t \bar{t}$ threshold, decays to $H_{5}^{0} Z$ and $H_{5}^{ \pm} W^{\mp}$ can be significant, but improvements to the handling of near-threshold decays in GMCALC are needed to fully explore the branching ratios in this region. $H_{3}^{+}$decays predominantly to $t \bar{b}$ for $m_{5}$ values up to about $500 \mathrm{GeV}$, where $h W^{+}$takes over as the dominant decay mode.

We note that the decays $H_{3}^{0} \rightarrow h Z, H_{3}^{ \pm} \rightarrow h W^{ \pm}$, and $H \rightarrow h h$ are of particular phenomenological interest, since the partial widths of such decays are not usually large in other extended Higgs models.

In addition to the phenomenological predictions above, we have implemented numerical integration procedures into GMCALC 1.4 .0 to enable more efficient computation of offshell $H_{i} \rightarrow V_{1}^{*} V_{2}^{*}$ decay processes. The original routine used rectangular integration, with $1,000 \times 1,000$ points for $m_{H_{i}} \leq M_{V_{1}}+M_{V_{2}}$ and $100 \times 100$ points for $m_{H_{i}}>M_{V_{1}}+M_{V_{2}}$. This routine was compared to a VEGAS routine and GaussLegendre quadrature. Since the integral can be done either with respect to the integration variables $Q_{i}$ or $\rho_{i}$, the integrands and integration regions for $Q_{i}$ and $\rho_{i}$ were analyzed, and it was determined that $Q_{i}$ was a better choice for integration when $m_{H_{i}} \leq M_{V_{1}}+M_{V_{2}}$, while $\rho_{i}$ was optimal for $m_{H_{i}}>M_{V_{1}}+M_{V_{2}}$. The new numerical integration routines were then tested, using Higgs decay partial width values computed by HDECAY 6.42 in the range $70 \mathrm{GeV} \leq m_{H_{i}} \leq 300 \mathrm{GeV}$ as the standard for comparison.

Gauss-Legendre quadrature was chosen for $m_{H_{i}}>M_{V_{1}}+M_{V_{2}}$, since the $\rho_{i}$ integrand is relatively well-behaved in that range, allowing the Gauss-Legendre routine to compute the integral accurately with relatively few points. 10-point and 16-point Gauss-Legendre quadrature routines were tested for accuracy, and the 16-point routine was consistently more accurate than the original routine in the $m_{H_{i}}>M_{V_{1}}+M_{V_{2}}$ range. 
We then tested an implementation of the VEGAS algorithm [23], which we borrowed with permission from PROSPINO [24], in the range $m_{H_{i}} \leq M_{V_{1}}+M_{V_{2}}$. We chose $Q_{i}$ as the variable of integration, since the VEGAS routine is not effective at integrating the thin, oddly-shaped integration region that $\rho_{i}$ has. We then took a variety of values for the number of sampling points and the number of sampling iterations in the VEGAS routine, and compared the results to the HDECAY data, the original routine, and a run of the VEGAS routine computed with 1,000, 000 sampling points and 30 iterations. We determined that the accuracy of the routine levelled off at around 10,000 sampling points and 3 iterations, where it showed a maximum deviation of $\sim 0.8 \%$ relative to the original routine, and $\sim 0.3 \%$ relative to the highaccuracy run of the VEGAS routine. Using an Intel Celeron CPU N2840 with a base frequency of $2.16 \mathrm{GHz}$ to calculate runtimes on a single core, we found that the VEGAS routine, with 10,000 sampling points and 3 iterations, was able to calculate the offshell integrals from $m_{H_{i}}=70 \mathrm{GeV}$ to $m_{H_{i}}=300 \mathrm{GeV}$, in increments of $1 \mathrm{GeV}$, in 12 seconds, a significant speedup compared to 152 seconds for the original routine.

Finally, we combined the Gauss-Legendre and VEGAS routines, using GaussLegendre for $m_{H_{i}}>M_{V_{1}}+M_{V_{2}}$ and VEGAS for $m_{H_{i}} \leq M_{V_{1}}+M_{V_{2}}$. The resulting routine was about as accurate as the original routine, as can be seen from Figs. 52 and 53. A runtime test showed that the new, combined routine was 23 times faster than the original for $m_{H_{i}} \leq M_{V_{1}}+M_{V_{2}}$, and 41 times faster than the original for $m_{H_{i}}>M_{V_{1}}+M_{V_{2}}$. 


\section{References}

[1] H. E. Logan and M. B. Reimer, "Characterizing a benchmark scenario for heavy Higgs boson searches in the Georgi-Machacek model," Phys. Rev. D96, 095029 (2017) [arXiv:1709.01883[hep-ph]]

[2] J.F. Donoghue E. Golowich, B.R. Holstein, Dynamics of the Standard Model (Cambridge University Press, Great Britain, 1992).

[3] G. Aad et al. [ATLAS Collaboration], "Observation of a new particle in the search for the Standard Model Higgs boson with the ATLAS detector at the LHC," Phys. Lett. B 716, 1 (2012) [arXiv:1207.7214 [hep-ex]]; S. Chatrchyan et al. [CMS Collaboration], "Observation of a new boson at a mass of $125 \mathrm{GeV}$ with the CMS experiment at the LHC," Phys. Lett. B 716, 30 (2012) [arXiv:1207.7235 [hep-ex]].

[4] H. Georgi and M. Machacek, "Doubly Charged Higgs Bosons," Nucl. Phys. B 262, 463 (1985).

[5] M. S. Chanowitz and M. Golden, "Higgs Boson Triplets With $\mathrm{M}(W)=\mathrm{M}(Z)$ $\cos \theta_{W}, "$ Phys. Lett. 165B, 105 (1985).

[6] K. Hartling, K. Kumar and H. E. Logan, "The decoupling limit in the GeorgiMachacek model," Phys. Rev. D 90, no. 1, 015007 (2014) [arXiv:1404.2640 [hep$\mathrm{ph}]]$.

[7] M. Aoki and S. Kanemura, "Unitarity bounds in the Higgs model including triplet fields with custodial symmetry," Phys. Rev. D 77, no. 9, 095009 (2008) [Erratum: Phys. Rev. D 89, no. 5, 059902 (2014)] [arXiv:0712.4053 [hep-ph]].

[8] K. Hartling, K. Kumar and H. E. Logan, "GMCALC: a calculator for the GeorgiMachacek model," arXiv:1412.7387 [hep-ph]. 
[9] D. de Florian et al. [LHC Higgs Cross Section Working Group], "Handbook of LHC Higgs Cross Sections: 4. Deciphering the Nature of the Higgs Sector," arXiv:1610.07922 [hep-ph].

[10] K. Hartling, K. Kumar and H. E. Logan, "Indirect constraints on the GeorgiMachacek model and implications for Higgs boson couplings," Phys. Rev. D 91, no. 1, 015013 (2015) [arXiv:1410.5538 [hep-ph]].

[11] V. Khachatryan et al. [CMS Collaboration], "Study of vector boson scattering and search for new physics in events with two same-sign leptons and two jets," Phys. Rev. Lett. 114, no. 5, 051801 (2015) [arXiv:1410.6315 [hep-ex]].

[12] C. W. Chiang, S. Kanemura and K. Yagyu, "Novel constraint on the parameter space of the Georgi-Machacek model with current LHC data," Phys. Rev. D 90, no. 11, 115025 (2014) [arXiv:1407.5053 [hep-ph]].

[13] G. Aad et al. [ATLAS Collaboration], "Evidence for Electroweak Production of $W^{ \pm} W^{ \pm} j j$ in $p p$ Collisions at $\sqrt{s}=8 \mathrm{TeV}$ with the ATLAS Detector," Phys. Rev. Lett. 113, no. 14, 141803 (2014) [arXiv:1405.6241 [hep-ex]].

[14] G. Aad et al. [ATLAS Collaboration], "Search for a Charged Higgs Boson Produced in the Vector-Boson Fusion Mode with Decay $H^{ \pm} \rightarrow W^{ \pm} Z$ using $p p$ Collisions at $\sqrt{s}=8 \mathrm{TeV}$ with the ATLAS Experiment," Phys. Rev. Lett. 114, no. 23, 231801 (2015) [arXiv:1503.04233 [hep-ex]].

[15] A. M. Sirunyan et al. [CMS Collaboration], "Search for charged Higgs bosons produced in vector boson fusion processes and decaying into a pair of $\mathrm{W}$ and Z bosons using proton-proton collisions at $\operatorname{sqrt}(\mathrm{s})=13 \mathrm{TeV}$," arXiv:1705.02942 [hep-ex].

[16] M. Zaro and H. Logan, "Recommendations for the interpretation of LHC searches for $H_{5}^{0}, H_{5}^{ \pm}$, and $H_{5}^{ \pm \pm}$in vector boson fusion with 
decays to vector boson pairs," LHCHXSWG-2015-001, available from https://cds.cern.ch/record/2002500.

[17] C. W. Chiang, A. L. Kuo and T. Yamada, "Searches of exotic Higgs bosons in general mass spectra of the Georgi-Machacek model at the LHC," JHEP 1601, 120 (2016) [arXiv:1511.00865 [hep-ph]].

[18] A. David et al. [LHC Higgs Cross Section Working Group], "LHC HXSWG interim recommendations to explore the coupling structure of a Higgs-like particle," arXiv:1209.0040 [hep-ph].

[19] G. Aad et al. [ATLAS and CMS Collaborations], "Measurements of the Higgs boson production and decay rates and constraints on its couplings from a combined ATLAS and CMS analysis of the LHC $p p$ collision data at $\sqrt{\mathrm{s}}=7$ and 8 TeV," JHEP 1608, 045 (2016) [arXiv:1606.02266 [hep-ex]].

[20] LHC Higgs Cross Section Working Group, "BSM Higgs production cross sections at $\sqrt{s}=13 \mathrm{TeV}$ (update in CERN Report 4 2016)," https://twiki.cern.ch/twiki/bin/view/LHCPhysics/CERNYellowReportPageBSMAt13TeV

[21] A. Bredenstein, A. Denner, S. Dittmaier and M.M. Weber, "Precise predictions for the Higgs-boson decay $H \rightarrow W W / Z Z \rightarrow 4$ leptons," Phys. Rev. D 74, 013004 (2006) [arXiv:hep-ph/0604011].

[22] A. Djouadi, J. Kalinowski and M. Spira, "HDECAY: A Program for Higgs boson decays in the standard model and its supersymmetric extension," Comput. Phys. Commun. 108, 56 (1998) [arXiv:hep-ph/9704448].

[23] G. P. Lepage, "Vegas: An Adaptive Multidimensional Integration Program," CLNS-80/447. 
[24] W. Beenakker, R. Hopker and M. Spira, "PROSPINO: A Program for the production of supersymmetric particles in next-to-leading order QCD," hep$\mathrm{ph} / 9611232$. 


\section{Appendices}

\section{Appendix A: Gauss-Legendre quadrature}

Let us suppose that we wish to compute the value of an integral,

$$
\int_{a}^{b} f(x) d x
$$

through numerical approximation. In general, numerical integration takes the following format:

$$
\int_{a}^{b} f(x) d x \approx \sum_{i=1}^{n} w_{i} f\left(x_{i}\right)
$$

where the points sampled $x_{i}$ are known as nodes and the coefficients $w_{i}$ are known as weights. A naive method of integration, the midpoint rule, divides the integration region into $n$ equally sized subregions, takes $x_{i}$ to be the midpoints of these regions, and applies the uniform weight $w_{i}=(b-a) / n$ to all of the sample points:

$$
\int_{a}^{b} f(x) d x \approx \Delta x \sum_{i=1}^{n} f(a+(i-1 / 2) \Delta x), \quad \Delta x=\frac{b-a}{n}
$$

(The original integration routine in GMCALC 1.2.1 used the midpoint rule in its inte-

gral computations.) The midpoint rule works by assuming that, over short intervals at least, the integrand $f(x)$ is well-approximated by a straight line. However, can we do better? Is there a choice of nodes and weights which has provably better accuracy when applied to arbitrary well-behaved integrals?

The Gauss-Legendre quadrature method was designed with this question in mind. Since a lot of well-behaved functions can be approximated using polynomials, Gauss-Legendre quadrature chooses $n$ nodes $x_{i}$ and $n$ weights $w_{i}$ such that the integral of any polynomial of degree $\leq 2 n-1$ will be evaluated exactly.

For simplicity, the integration region is assumed to be $[-1,+1]$; if this is not true, 
we can always rescale the integration region by performing the change of variables

$$
x=\frac{b-a}{2} y+\frac{a+b}{2}, \quad y \in[-1,+1]
$$

Once the integration region has been rescaled, we must then find the nodes $x_{i}$ and weights $w_{i}$ which satisfy the following system of equations:

$$
\sum_{i=1}^{n} w_{i} x_{i}^{k}=\int_{-1}^{+1} x^{k} d x=\left\{\begin{array}{ll}
\frac{2}{k+1} & k \text { even } \\
0 & k \text { odd }
\end{array}, \quad k=0,1,2, \ldots 2 n-1\right.
$$

For $n=1$, the equations are

$$
\begin{aligned}
& w_{1} \cdot 1=2, \\
& w_{1} \cdot x_{1}=0,
\end{aligned}
$$

and the solution is $w_{1}=2, x_{1}=0$; thus, Gauss-Legendre quadrature with one point is equivalent to the midpoint rule. For $n=2$, the equations are

$$
\begin{aligned}
& w_{1}+w_{2}=2, \\
& w_{1} x_{1}+w_{2} x_{2}=0, \\
& w_{1} x_{1}^{2}+w_{2} x_{2}^{2}=\frac{2}{3}, \\
& w_{1} x_{1}^{3}+w_{2} x_{2}^{3}=0,
\end{aligned}
$$

and the solution is $x_{1}=-1 / \sqrt{3}, x_{2}=+1 / \sqrt{3}, w_{1}=w_{2}=1$. More generally, it has been proven that, for $n$-point Gauss-Legendre quadrature, the nodes $x_{i}$ are the roots of the Legendre polynomial $P_{n}(x)$, and the weights are equal to

$$
w_{i}=\frac{2}{\left(1-x_{i}^{2}\right)\left[P_{n}^{\prime}\left(x_{i}\right)\right]^{2}} .
$$




\begin{tabular}{|c|c|}
\hline$x_{i}$ & $w_{i}$ \\
\hline \pm 0.0950125 & 0.1894506 \\
\pm 0.2816036 & 0.1826034 \\
\pm 0.4580168 & 0.1691565 \\
\pm 0.6178762 & 0.1495960 \\
\pm 0.7554044 & 0.1246290 \\
\pm 0.8656312 & 0.0951585 \\
\pm 0.9445750 & 0.0622535 \\
\pm 0.9894009 & 0.0271525 \\
\hline
\end{tabular}

Table 5: The approximate values of the nodes and weights used in 16-point GaussLegendre quadrature.

We have listed the approximate values of the nodes and weights for 16-point GaussLegendre quadrature in Table 5.

The Legendre polynomials are a sequence of polynomials which are the solutions of the differential equations

$$
\frac{d}{d x}\left[\left(1-x^{2}\right) \frac{d P_{n}(x)}{d x}\right]+n(n+1) P_{n}(x)=0, \quad P_{n}(1)=1, \quad n=0,1,2, \ldots
$$

The orthogonality of the Legendre polynomials,

$$
\int_{-1}^{+1} P_{m}(x) P_{n}(x) d x=\frac{2}{2 n+1} \delta_{m n}
$$

is the reason for their appearance here. 